Supporting Information

\title{
Synthesis of Functionalized Organotrifluoroborates via Halomethyltrifluoroborates
}

\author{
Gary A. Molander* and Jungyeob Ham \\ Roy and Diana Vagelos Laboratories, Department of Chemistry \\ University of Pennsylvania, Philadelphia, Pennsylvania 19104-6323 \\ gmolandr@sas.upenn.edu
}

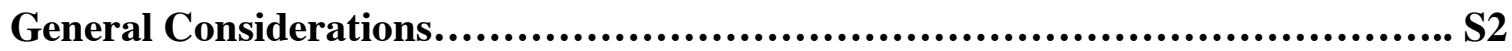

Preparation of Potassium Halomethyltrifluoroborates 1 and 2 ..................... S2

Reaction Condition A for Preparing Compounds 3-5 ............................... S3

Reaction Condition B for Preparing Compounds 6-9.................................. S4

Reaction Condition C for Preparing Compounds 10 and 11.......................... S5

Reaction Condition D for Preparing Compound 12 ................................ S6

Reaction Condition E for Preparing Compound 13................................... S6

Reaction Condition F for Preparing Compounds 14-16............................. S7

Reaction Condition for Preparing Compounds 17 and 18.......................... S8

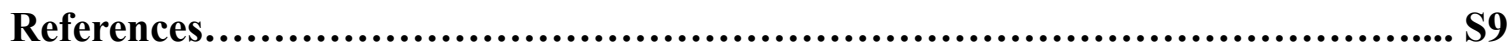

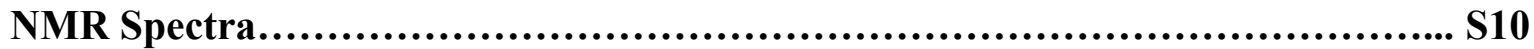


General Considerations: ${ }^{1} \mathrm{H}-,{ }^{13} \mathrm{C}$-, and ${ }^{19} \mathrm{~F}-\mathrm{NMR}$ spectra were recorded on 500.39 or $360.13,125.75$, and $470.55 \mathrm{MHz}$, respectively. ${ }^{19} \mathrm{~F}$-NMR chemical shifts were referenced to external $\mathrm{CFCl}_{3}(0.0 \mathrm{ppm}) .{ }^{11} \mathrm{~B}-\mathrm{NMR}$ spectra at 128.4 MHz were obtained on a spectrometer equipped with the appropriate decoupling accessories. All ${ }^{11} \mathrm{~B}$-chemical shifts were referenced to external $\mathrm{BF}_{3} \cdot \mathrm{OEt}_{2}(0.0 \mathrm{ppm})$ with a negative sign indicating an upfield shift. Mass spectra of potassium organotrifluoroborates were performed using negative ion electrospray at the mass spectrometry facilities at the University of Pennsylvania. Elemental analyses were performed by MHW Laboratories. THF was distilled from sodium. Commercially obtained anhydrous DMSO, DMF, and reagents were used without further purification.

\section{Preparation of Potassium Halomethyltrifluoroborates 1 and 2.}

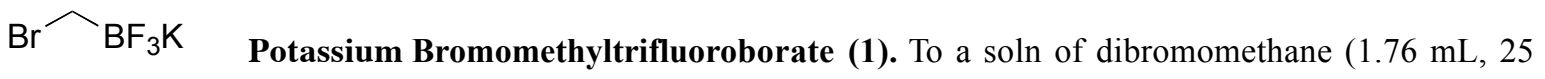
mmol) and triisopropyl borate $(5.2 \mathrm{~mL}, 23 \mathrm{mmol})$ in THF $(35 \mathrm{~mL})$ was slowly added $n$-BuLi $(2.5 \mathrm{M}$ soln in hexanes, $8.5 \mathrm{~mL}, 21 \mathrm{mmol}$ ) at $-78{ }^{\circ} \mathrm{C}$ for $1 \mathrm{~h}$ under $\mathrm{N}_{2}$. The soln was vigorously stirred for $1 \mathrm{~h}$. Then, $\mathrm{KHF}_{2}$ ( $4.9 \mathrm{~g}, 63 \mathrm{mmol}$ ) was added in one portion, followed by the dropwise addition of $\mathrm{H}_{2} \mathrm{O}(15 \mathrm{~mL})$. The reaction mixture was warmed to $\mathrm{rt}$. After stirring at $\mathrm{rt}$ for $30 \mathrm{~min}$, the suspension was concentrated on a rotary evaporator. The resulting white solids were subjected to high vacuum for $3 \mathrm{~h}$. The crude compound was purified by dissolving in acetone and precipitating with $\mathrm{Et}_{2} \mathrm{O}$ to obtain the desired compound $\mathbf{1}$ as a white solid $(3.75 \mathrm{~g}, 89 \%$ yield $) . \mathrm{mp}=220{ }^{\circ} \mathrm{C} .{ }^{1} \mathrm{H}$ NMR $\left(500 \mathrm{MHz}\right.$, Acetone- $\left.d_{6}\right) \delta 2.21$ (br s, 2H). ${ }^{13} \mathrm{C}$ NMR $(125.8$ MHz, Acetone- $\left.d_{6}\right) \delta$ no peaks. ${ }^{19} \mathrm{~F}\left(470.8 \mathrm{MHz}\right.$, Acetone- $\left.d_{6}\right) \delta$-146.2. ${ }^{11} \mathrm{~B}$ NMR (128.4 MHz, Acetone- $\left.d_{6}\right) \delta$ 2.95. IR (neat): $3435,1696,1256,1096,980,952 \mathrm{~cm}^{-1}$. HRMS $(\mathrm{m} / \mathrm{z})$ : calcd. for $\mathrm{C}_{1} \mathrm{H}_{2} \mathrm{BBrF}_{3}, 160.9385$ [M$\mathrm{K}^{+}$; found, 160.9383 .

$\mathrm{BF}_{3} \mathrm{~K}$ Potassium Iodomethyltrifluoroborate (2). The compound was prepared using diiodomethane by the procedure used for potassium bromomethyltrifluoroborate $\left(4.6 \mathrm{~g}, 89 \%\right.$ yield). $\mathrm{mp}=242{ }^{\circ} \mathrm{C} .{ }^{1} \mathrm{H} \mathrm{NMR}$ $\left(500 \mathrm{MHz}\right.$, Acetone- $\left.d_{6}\right) \delta 1.80$ (br s, $\left.2 \mathrm{H}\right) .{ }^{13} \mathrm{C}$ NMR (125.8 MHz, Acetone- $\left.d_{6}\right) \delta$ no peaks. ${ }^{19} \mathrm{~F}(470.8 \mathrm{MHz}$, Acetone- $\left.d_{6}\right) \delta$-145.0. ${ }^{11} \mathrm{~B}$ NMR (128.4 MHz, Acetone- $\left.d_{6}\right) \delta$ 2.97. IR (neat): 3435, 1398, 1165, 1073, 953, 931 $\mathrm{cm}^{-1}$. HRMS $(\mathrm{m} / \mathrm{z})$ : calcd. for $\mathrm{C}_{1} \mathrm{H}_{2} \mathrm{BF}_{3} \mathrm{I}, 208.9246\left[\mathrm{M}-\mathrm{K}^{+}\right]$; found, 208.9254 .

$\widehat{\mathrm{BF}_{3} \mathrm{~K}}$ Potassium Iodomethyltrifluoroborate (2). To a soln of potassium bromomethyltrifluoroborate $(2.0 \mathrm{~g}, 10 \mathrm{mmol})$ in acetone $(150 \mathrm{~mL})$ was added $\mathrm{NaI}(1.5 \mathrm{~g}, 10 \mathrm{mmol})$ in one portion. After stirring at $\mathrm{rt}$ for 2 $\mathrm{h}$, the suspension was filtered through Celite and then the solvent was evaporated under reduced pressure to give the crude product. The crude compound was purified by dissolving in a minimal amount of dry acetone 
and precipitating with $\mathrm{Et}_{2} \mathrm{O}$ to obtain the pure compound 2 as a white solid ( $2.38 \mathrm{~g} 96 \%$ yield).

\section{Reaction Condition A for Preparing Compounds 3-5.}

To a soln of an organolithium or Grignard reagent $(1.2 \mathrm{mmol})$ in anhydrous THF $(2 \mathrm{~mL})$ was slowly added a soln of potassium iodomethyltrifluoroborate $(99.1 \mathrm{mg}, 0.4 \mathrm{mmol})$ in THF $(3 \mathrm{~mL})$ at $-25{ }^{\circ} \mathrm{C}$ for $10 \mathrm{~min}$ under $\mathrm{N}_{2}$ and then the reaction mixture was slowly warmed to $0{ }^{\circ} \mathrm{C}$ during the reaction time (see Table 2). After the reaction was complete, it was quenched with $1.5 \mathrm{~N} \mathrm{KHF}_{2}(2 \mathrm{~mL})$ followed by removal of all the solvent under high vacuum. The residual solid was dissolved in dry acetone $(15 \mathrm{~mL})$, the insoluble salts were filtered off and then the product was concentrated. The crude solid was purified by dissolving in a minimal amount of dry acetone $(4 \mathrm{~mL})$ and precipitating with $\mathrm{Et}_{2} \mathrm{O}(10 \mathrm{~mL})$ to obtain the desired pure compound.

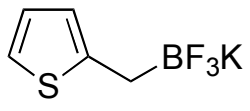

Potassium (2-Thiophenyl)methyltrifluoroborate (3). The title compound was isolated as a yellow solid (70 mg, 86\% yield) using 2-thienyllithium (1.0 M soln in THF). mp $=172-173{ }^{\circ} \mathrm{C}\left(\mathrm{dec}\right.$.). ${ }^{1} \mathrm{H}$ NMR $\left(500 \mathrm{MHz}\right.$, Acetone- $\left.d_{6}\right) \delta 6.85(\mathrm{dd}, 1 \mathrm{H}, J=5.2,1.2 \mathrm{~Hz}), 6.72(\mathrm{dd}, 1 \mathrm{H}, J=5.2,3.3 \mathrm{~Hz}), 6.59(\mathrm{dd}, 1 \mathrm{H}, J$ $=3.3,0.9 \mathrm{~Hz}), 1.78(\mathrm{br} \mathrm{s}, 2 \mathrm{H}) .{ }^{13} \mathrm{C}$ NMR $\left(125.8 \mathrm{MHz}\right.$, Acetone- $\left.d_{6}\right) \delta 125.8,122.2,119.5,110.3 .{ }^{19} \mathrm{~F}(470.8$ MHz, Acetone- $\left.d_{6}\right) \delta-141.8 .{ }^{11} \mathrm{~B}$ NMR (128.4 MHz, Acetone- $\left.d_{6}\right) \delta$ 4.18. IR (neat): 3531, 2886, 1609, 1532, 1443, 1234, 1077, 1034, 977, $945 \mathrm{~cm}^{-1}$. $\operatorname{HRMS}(\mathrm{m} / \mathrm{z})$ : calcd. for $\mathrm{C}_{5} \mathrm{H}_{5} \mathrm{BF}_{3} \mathrm{~S}, 165.0157\left[\mathrm{M}-\mathrm{K}^{+}\right]$; found, 165.0161 .

$\mathrm{BF}_{3} \mathrm{~K}$ Potassium Pentyltrifluoroborate (4). The title compound was isolated as a white solid (59 mg, 83\% yield) using $n$-BuLi (2.5 M soln in hexanes). mp $>250{ }^{\circ} \mathrm{C} .{ }^{1} \mathrm{H}$ NMR (500 MHz, Acetone- $\left.d_{6}\right) \delta$ $1.25(\mathrm{~m}, 6 \mathrm{H}), 0.84$ (t, 3H, $J=6.8 \mathrm{~Hz}), 0.13$ (br s, 2H). ${ }^{13} \mathrm{C}$ NMR $\left(125.8 \mathrm{MHz}\right.$, Acetone- $\left.d_{6}\right) \delta$ 36.3, 25.6, 23.1, 14.0. ${ }^{19} \mathrm{~F}\left(470.8 \mathrm{MHz}\right.$, Acetone- $\left.d_{6}\right) \delta$-141.6. ${ }^{11} \mathrm{~B}$ NMR (128.4 MHz, Acetone- $\left.d_{6}\right) \delta$ 5.85. IR (neat): 3391, 2916, 1460, 1070, 971, $890 \mathrm{~cm}^{-1}$. HRMS $(\mathrm{m} / \mathrm{z})$ : calcd. for $\mathrm{C}_{5} \mathrm{H}_{11} \mathrm{BF}_{3}, 139.0906\left[\mathrm{M}-\mathrm{K}^{+}\right]$; found, 139.0905 .

$\mathrm{BF}_{3} \mathrm{~K}$ Potassium 1-Propenyltrifluoroborate (5). ${ }^{1}$ The title compound was isolated as a white solid (55 mg, 85\% yield) using allylmagnesium chloride (2.0 M soln in THF). $\mathrm{mp}=243-245{ }^{\circ} \mathrm{C}(\mathrm{dec}.) .{ }^{1} \mathrm{H}$ NMR (500 MHz, Acetone- $\left.d_{6}\right) \delta 5.89(\mathrm{~m}, 1 \mathrm{H}), 4.83(\mathrm{~m}, 1 \mathrm{H}), 4.67(\mathrm{~m}, 1 \mathrm{H}), 1.96(\mathrm{~m}, 2 \mathrm{H}), 0.21(\mathrm{q}, 2 \mathrm{H}, J=7.1$ Hz). ${ }^{13} \mathrm{C}$ NMR $\left(125.8 \mathrm{MHz}\right.$, Acetone- $\left.d_{6}\right) \delta 144.8,109.8,30.1 .{ }^{19} \mathrm{~F}\left(470.8 \mathrm{MHz}\right.$, Acetone- $\left.d_{6}\right) \delta$-141.9. ${ }^{11} \mathrm{~B}$ NMR (128.4 MHz, Acetone- $d_{6}$ ) $\delta$ 5.36. IR (neat): 3468, 2911, 1701, 1638, 1445, 1253, 1081, 1006, 962, 902 , $863 \mathrm{~cm}^{-1}$. HRMS $(\mathrm{m} / \mathrm{z})$ : calcd. for $\mathrm{C}_{4} \mathrm{H}_{7} \mathrm{BF}_{3}, 123.0592\left[\mathrm{M}-\mathrm{K}^{+}\right]$, found, 123.0584 . 


\section{Reaction Condition B for Preparing Compounds 6-9.}

Potassium bromomethyltrifluoroborate $(100 \mathrm{mg}, 0.5 \mathrm{mmol})$ was added to neat alkylamine $(2 \mathrm{~mL})$ at $\mathrm{rt}$. The reaction mixture was heated at $80{ }^{\circ} \mathrm{C}$ for $30 \mathrm{~min}$ and concentrated under high vacuum. The residual product was dissolved in a soln of $\mathrm{KHCO}_{3}(69.1 \mathrm{mg}, 0.5 \mathrm{mmol})$ and dry acetone $(15 \mathrm{~mL})$, followed by stirring the mixture at rt. After $20 \mathrm{~min}$, the insoluble salts were filtered off, and then the product was concentrated. The crude solid was purified by dissolving in a minimal amount of dry acetone and precipitating with $\mathrm{Et}_{2} \mathrm{O}$ to obtain the desired pure compound.

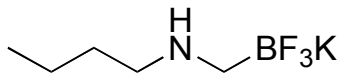

Potassium $N$-Butyl-aminomethyltrifluoroborate (6). The title compound was isolated as a yellow solid (85 mg, $88 \%$ yield) using $n$-butylamine. $\mathrm{mp}=118-120{ }^{\circ} \mathrm{C} .{ }^{1} \mathrm{H}$ NMR $(500 \mathrm{MHz}$, Acetone- $d_{6}$ ) $\delta 6.08$ (br s, $\left.1 \mathrm{H}, \mathrm{NH}\right), 3.13(\mathrm{~m}, 2 \mathrm{H}), 2.00$ (br s, $\left.2 \mathrm{H},-\mathrm{CH}_{2}-\mathrm{B}\right), 1.80(\mathrm{~m}, 2 \mathrm{H}), 1.44(\mathrm{~m}, 2 \mathrm{H}), 0.95(\mathrm{t}$, $3 \mathrm{H}, J=7.4 \mathrm{~Hz}) .{ }^{13} \mathrm{C}$ NMR $\left(125.8 \mathrm{MHz}\right.$, Acetone- $\left.d_{6}\right) \delta 50.0,27.9,19.5,13.0 .{ }^{19} \mathrm{~F}\left(470.8 \mathrm{MHz}\right.$, Acetone- $\left.d_{6}\right) \delta$ 145.7. ${ }^{11}$ B NMR (128.4 MHz, Acetone- $d_{6}$ ) $\delta$ 2.31. IR (neat): 3400, 3003, 2821, 2443, 1471, 1103, 1024, 1010, $958,868 \mathrm{~cm}^{-1}$. HRMS $(\mathrm{m} / \mathrm{z})$ : calcd. for $\mathrm{C}_{5} \mathrm{H}_{12} \mathrm{BF}_{3} \mathrm{~N}, 154.1015\left[\mathrm{M}-\mathrm{K}^{+}\right]$; found, 154.1019.

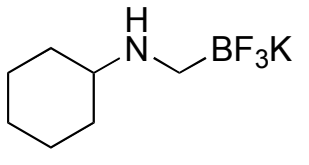

Potassium $N$-Cyclohexyl-aminomethyltrifluoroborate (7). The title compound was isolated as a white solid (107 mg, 98\% yield) using cyclohexylamine. $\mathrm{mp}=228{ }^{\circ} \mathrm{C} .{ }^{1} \mathrm{H}$ NMR $(500 \mathrm{MHz}$, Acetone- $\left.d_{6}\right) \delta 6.74(\mathrm{br} \mathrm{s}, 1 \mathrm{H}, \mathrm{NH}), 3.12(\mathrm{tt}, 1 \mathrm{H}, J=11.5,3.8 \mathrm{~Hz}), 2.25(\mathrm{ddd}, 2 \mathrm{H}, J=11.2,2.4,1.4 \mathrm{~Hz}), 1.97$ (br s, 2H, $\left.-\mathrm{CH}_{2}-\mathrm{B}\right), 1.87$ (dt, 2H, $\left.J=14.0,3.5 \mathrm{~Hz}\right), 1.69(\mathrm{~m}, 1 \mathrm{H}), 1.48(\mathrm{qd}, 2 \mathrm{H}, J=12.5,3.4 \mathrm{~Hz}), 1.36(\mathrm{qt}, 2 \mathrm{H}$, $J=12.7,3.2 \mathrm{~Hz}$ ), 1.20 (qt, $1 \mathrm{H}, J=12.8,3.5 \mathrm{~Hz}) .{ }^{13} \mathrm{C}$ NMR $\left(125.8 \mathrm{MHz}\right.$, DMSO- $d_{6}$ ) $\delta 58.0,29.0,25.7,24.9$. ${ }^{19} \mathrm{~F}\left(470.8 \mathrm{MHz}\right.$, Acetone- $\left.d_{6}\right) \delta$-145.7. ${ }^{11} \mathrm{~B}$ NMR (128.4 MHz, Acetone- $\left.d_{6}\right) \delta$ 2.11. IR (neat): 3229, 2927 , 2856, 1585, 1445, 1298, 1067, 1023, 900, $826 \mathrm{~cm}^{-1}$. HRMS $(\mathrm{m} / \mathrm{z})$ : calcd. for $\mathrm{C}_{7} \mathrm{H}_{14} \mathrm{BF}_{3} \mathrm{~N}, 180.1171\left[\mathrm{M}-\mathrm{K}^{+}\right]$; found, 180.1174 .

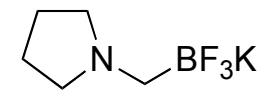

Potassium Pyrrolidine-1-methyltrifluoroborate (8). The title compound was isolated as a yellow solid ( $88 \mathrm{mg}, 92 \%$ yield) using pyrrolidine. $\mathrm{mp}=85-87^{\circ} \mathrm{C} .{ }^{1} \mathrm{H}$ NMR $\left(500 \mathrm{MHz}\right.$, Acetone- $\left.d_{6}\right) \delta 3.40$ (br s, 4H, $-\left(\mathrm{CH}_{2}\right)_{2}-\mathrm{N}$ ), 2.17 (br s, 2H, $\left.-\mathrm{CH}_{2}-\mathrm{B}\right), 2.09$ (m, 4H). ${ }^{13} \mathrm{C}$ NMR (125.8 MHz, Acetone- $d_{6}$ ) $\delta$ 56.1, 22.7. ${ }^{19} \mathrm{~F}\left(470.8 \mathrm{MHz}\right.$, Acetone- $\left.d_{6}\right) \delta-144.3 .{ }^{11} \mathrm{~B}$ NMR (128.4 MHz, Acetone- $\left.d_{6}\right) \delta$ 1.98. IR (neat): 3413, 3208, 2974, 2761, 1631, 1460, 1309, 1178, $1005 \mathrm{~cm}^{-1}$. HRMS $(\mathrm{m} / z)$ : calcd. for $\mathrm{C}_{5} \mathrm{H}_{10} \mathrm{BF}{ }_{3} \mathrm{~N}, 152.0858\left[\mathrm{M}-\mathrm{K}^{+}\right]$; found, 152.0848 . 
$\checkmark \mathrm{N}_{\sim} \mathrm{BF}_{3} \mathrm{~K}$

Potassium Piperidinyl-1-methyltrifluoroborate (9). The title compound was isolated as a white solid (97 mg, 95\% yield) using piperidine. $\mathrm{mp}=146-148{ }^{\circ} \mathrm{C} .{ }^{1} \mathrm{H}$ NMR $\left(500 \mathrm{MHz}\right.$, Acetone- $\left.d_{6}\right) \delta 3.28$ (br s, 4H, - $\left.\left(\mathrm{CH}_{2}\right)_{2}-\mathrm{N}\right), 2.06$ (br s, $\left.2 \mathrm{H},-\mathrm{CH}_{2}-\mathrm{B}\right), 1.89$ (m, 4H), 1.67, (br s, $\left.2 \mathrm{H},-\mathrm{CH}_{2}-\right) .{ }^{13} \mathrm{C}^{\mathrm{NMR}}(125.8 \mathrm{MHz}$, Acetone- $\left.d_{6}\right) \delta 56.4,24.0,22.4 .{ }^{19} \mathrm{~F}\left(470.8 \mathrm{MHz}\right.$, Acetone- $\left.d_{6}\right) \delta$-142.6. ${ }^{11} \mathrm{~B}$ NMR (128.4 MHz, Acetone- $\left.d_{6}\right) \delta$ 1.94 (q, $J=50.5 \mathrm{~Hz}$ ). IR (neat): 3401, 3180, 2951, 2774, 1458, 1306, 1227, 1043, 1004, $950 \mathrm{~cm}^{-1}$. HRMS $(\mathrm{m} / \mathrm{z})$ : calcd. for $\mathrm{C}_{6} \mathrm{H}_{12} \mathrm{BF}_{3} \mathrm{~N}, 166.1015$ [M-K ${ }^{+}$; found, 166.1016 .

\section{Reaction Condition C for Preparing Compounds 10 and 11.}

To a soln of the nucleophile $(1.2 \mathrm{mmol})$, which was prepared from substrate $(1.2 \mathrm{mmol})$ and $\mathrm{NaH}(1.2 \mathrm{mmol}$, $60 \%$ dispersion in mineral oil) in dry solvent $(3.5 \mathrm{~mL})$, was slowly added a soln of potassium iodomethyltrifluoroborate $(99.1 \mathrm{mg}, 0.4 \mathrm{mmol})$ in dry solvent $(2 \mathrm{~mL})$ at $0{ }^{\circ} \mathrm{C}$ for $0.5 \mathrm{~h}$ under $\mathrm{N}_{2}$. The reaction mixture was stirred at $\mathrm{rt}$ for $8 \mathrm{~h}$. After the reaction was completed, it was quenched with $1.5 \mathrm{~N} \mathrm{KHF}_{2}(2 \mathrm{~mL})$ followed by removal of all the solvent under high vacuum. The residual solid was dissolved in dry acetone $(15 \mathrm{~mL})$, the insoluble salts were filtered off, and then the product was concentrated. The crude solid was purified by dissolving in a minimal amount of dry acetone $(4 \mathrm{~mL})$ and precipitating with $\mathrm{Et}_{2} \mathrm{O}(10 \mathrm{~mL})$ to obtain the desired pure compound.

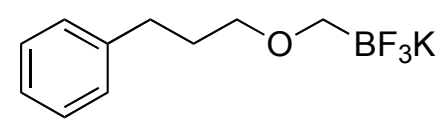

Potassium (4-Phenylpropoxy)methyltrifluoroborate (10). The title compound was isolated as a white solid ( $88 \mathrm{mg}, 86 \%$ yield) using 4-phenylpropanoxide. $\mathrm{mp}=220{ }^{\circ} \mathrm{C}$ (dec.). ${ }^{1} \mathrm{H}$ NMR $\left(500 \mathrm{MHz}\right.$, Acetone- $\left.d_{6}\right) \delta 7.25(\mathrm{~m}, 2 \mathrm{H}), 7.20(\mathrm{~m}, 2 \mathrm{H}), 7.14(\mathrm{~m}, 1 \mathrm{H}), 3.34(\mathrm{t}, 2 \mathrm{H}, J=6.9 \mathrm{~Hz}), 2.71$ $(\mathrm{q}, 2 \mathrm{H}, J=5.6 \mathrm{~Hz}), 2.61(\mathrm{t}, 2 \mathrm{H}, J=7.7 \mathrm{~Hz}), 1.85(\mathrm{~m}, 2 \mathrm{H}) .{ }^{13} \mathrm{C}$ NMR $\left(125.8 \mathrm{MHz}\right.$, Acetone- $\left.d_{6}\right) \delta 142.4,128.3$, 128.1, 125.5, 73.5, 32.2, 31.4. ${ }^{19} \mathrm{~F}\left(470.8 \mathrm{MHz}\right.$, Acetone- $\left.d_{6}\right) \delta$-146.2. ${ }^{11} \mathrm{~B}$ NMR (128.4 MHz, Acetone- $\left.d_{6}\right) \delta$ 3.26 (q, $J=54.9 \mathrm{~Hz}$ ). IR (neat): 3400, 2941, 2859, 1611, 1453, 1083, $996 \mathrm{~cm}^{-1}$. HRMS $(\mathrm{m} / \mathrm{z})$ : calcd. for $\mathrm{C}_{10} \mathrm{H}_{13} \mathrm{BF}_{3} \mathrm{O}, 217.1012\left[\mathrm{M}-\mathrm{K}^{+}\right]$; found, 217.1014.

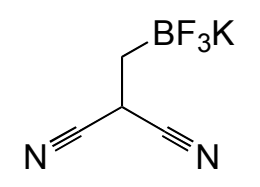

Potassium 2,2-Dicyanoethyltrifluoroborate (11). The title compound was isolated as a pink solid (68 mg, 91\% yield) using malononitrile. $\mathrm{mp}=223{ }^{\circ} \mathrm{C} .{ }^{1} \mathrm{H}$ NMR $\left(500 \mathrm{MHz}\right.$, Acetone- $\left.d_{6}\right) \delta 4.13(\mathrm{t}$, $1 \mathrm{H}, J=7.4 \mathrm{~Hz}$ ), 0.64 (br s, 2H). ${ }^{13} \mathrm{C}$ NMR $\left(125.8 \mathrm{MHz}\right.$, Acetone- $\left.d_{6}\right) \delta 108.9,48.4 .{ }^{19} \mathrm{~F}$ (470.8 MHz, Acetone$\left.d_{6}\right) \delta$-138.9. ${ }^{11} \mathrm{~B}$ NMR (128.4 MHz, Acetone- $\left.d_{6}\right) \delta 3.13$ (q, $J=54.9 \mathrm{~Hz}$ ). IR (neat): 2924, 2259, 2194, 1450, 
1250, 1034, 1014, 987, $967 \mathrm{~cm}^{-1}$. HRMS $(m / z)$ : calcd. for $\mathrm{C}_{4} \mathrm{H}_{3} \mathrm{BF}_{3} \mathrm{~N}_{2}, 147.0341$ [M-K ${ }^{+}$; found, 147.0341 .

\section{Reaction Condition D for Preparing Compound 12.}<smiles>CCOC(=O)CC(=O)CC[B-]Br</smiles>

Potassium Ethyl 3-Oxo-5-(trifluoroborato) pentanoate (12). A soln of ethyl acetoacetate dianion (1.2 mmol) was prepared by addition of ethyl acetoacetate $(0.15 \mathrm{~mL}, 1.2 \mathrm{mmol})$ to a soln of $\mathrm{NaH}(52 \mathrm{mg}, 1.3$ mmol, $60 \%$ dispersion in mineral oil) in THF $(2 \mathrm{~mL})$ at $0{ }^{\circ} \mathrm{C}$ for $20 \mathrm{~min}$ followed by addition of $n$-BuLi (2.5 M soln in hexanes, $0.48 \mathrm{~mL}, 1.2 \mathrm{mmol}$ ) at the same temperature under $\mathrm{N}_{2}$. The orange soln was stirred at $0{ }^{\circ} \mathrm{C}$ for an additional $15 \mathrm{~min}$. To this mixture was added a soln of potassium iodomethyltrifluoroborate $(99.1 \mathrm{mg}$, $0.4 \mathrm{mmol})$ in THF $(3 \mathrm{~mL})$ at $0{ }^{\circ} \mathrm{C}$. After $3 \mathrm{~h}$, it was quenched with $1.5 \mathrm{~N} \mathrm{KHF}_{2}(2 \mathrm{~mL})$ followed by removal of all the solvent under high vacuum. The residual solid was dissolved in hot EtOAc $(25 \mathrm{~mL})$, the insoluble salts were filtered off, and then the product was concentrated. The crude solid was purified by dissolution in a minimal amount of dry acetone and precipitation with $\mathrm{Et}_{2} \mathrm{O}$ to obtain the desired pure compound $\mathbf{1 2}$ as a yellow viscous solid (88 mg, 88\% yield). ${ }^{1} \mathrm{H}$ NMR (500 MHz, Acetone- $\left.d_{6}\right) \delta 4.10$ (q, $2 \mathrm{H}, J=7.1 \mathrm{~Hz}$, $\mathrm{CH}_{2} \mathrm{CH}_{3}$ ), 3.45 (s, $\left.2 \mathrm{H},-\mathrm{CO}-\mathrm{CH}_{2}-\mathrm{CO}-\right), 2.33$ (t, $2 \mathrm{H}, J=8.3 \mathrm{~Hz},-\mathrm{CO}-\mathrm{CH}_{2}-$ ), 1.21 (t, $3 \mathrm{H}, J=7.1 \mathrm{~Hz},-\mathrm{CH}_{2} \mathrm{CH}_{3}$ ), $0.36\left(\mathrm{~m}, 2 \mathrm{H},-\mathrm{CH}_{2}-\mathrm{B}\right) .{ }^{13} \mathrm{C}$ NMR $\left(125.8 \mathrm{MHz}\right.$, Acetone- $\left.d_{6}\right) \delta$ 206.9, 167.9, 60.3, 47.7, 40.0, 13.5. ${ }^{19} \mathrm{~F}(470.8$ MHz, Acetone- $\left.d_{6}\right) \delta-142.3 .{ }^{11} \mathrm{~B}$ NMR (128.4 MHz, Acetone- $d_{6}$ ) $\delta$ 5.26. IR (neat): 3436, 2914, 1731, 1370, 1312, 1234, 1072, $1024 \mathrm{~cm}^{-1}$. HRMS (m/z): calcd. for $\mathrm{C}_{7} \mathrm{H}_{10} \mathrm{BF}_{2} \mathrm{O}_{3}, 191.0691$ [M-KHF$\left.{ }^{+}\right]^{2}$; found, 191.0705.

\section{Reaction Condition E for Preparing Compound 13.}

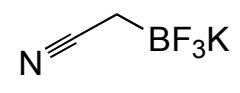

Potassium Cyanomethyltrifluoroborate (13). Potassium bromomethyltrifluoroborate (100 $\mathrm{mg}, 0.5 \mathrm{mmol}$ ) and potassium cyanide $(39.1 \mathrm{mg}, 0.6 \mathrm{mmol})$ were dissolved in DMSO $(3.5 \mathrm{~mL})$. The reaction mixture was heated at $80{ }^{\circ} \mathrm{C}$ for $10 \mathrm{~h}$ and concentrated under high vacuum. The residual product was dissolved in dry acetone $(15 \mathrm{~mL})$, the insoluble salts were filtered off, and then the product was concentrated. The crude solid was purified by dissolving in a minimal amount of dry acetone and precipitating with $\mathrm{Et}_{2} \mathrm{O}$ to obtain the desired pure compound 13 as a white solid (72 mg, 98\% yield). $\mathrm{mp}=146-147{ }^{\circ} \mathrm{C} .{ }^{1} \mathrm{H}$ NMR $(500$ MHz, Acetone- $\left.d_{6}\right) \delta 0.99(\mathrm{~m}, 2 \mathrm{H}) .{ }^{13} \mathrm{C}$ NMR $\left(125.8 \mathrm{MHz}\right.$, Acetone- $\left.d_{6}\right) \delta$ 124.6. ${ }^{19} \mathrm{~F}$ (470.8 MHz, Acetone- $\left.d_{6}\right)$ $\delta$-141.2. ${ }^{11}$ B NMR (128.4 MHz, Acetone- $d_{6}$ ) $\delta$ 2.83. IR (neat): 2238, 1197, 1004, $947 \mathrm{~cm}^{-1}$.

Anal. Calc for $\mathrm{C}_{2} \mathrm{H}_{2} \mathrm{BF}_{3} \mathrm{KN}$ : C, 16.35; H, 1.37; N, 9.53. Found: C, 16.50; H, 1.46; N, 9.81. 


\section{Reaction Condition F for Preparing Compounds 14-16.}

To a soln of aryl bromide (1.2 mmole) in anhydrous THF $(3 \mathrm{~mL})$ was added a soln of $t$-BuLi (1.5 M soln in pentane, $2.4 \mathrm{mmole}$ ) at $-78{ }^{\circ} \mathrm{C}$ for $15 \mathrm{~min}$ under $\mathrm{N}_{2}$. Sulfur powder (38.4 $\mathrm{mg}, 1.2 \mathrm{mmole}$ ) was added in one portion. After the reaction mixture changed to a yellow clear soln, a soln of potassium iodomethyltrifluoroborate $(99.1 \mathrm{mg}, 0.4 \mathrm{mmol})$ in THF $(3 \mathrm{~mL})$ was slowly added and then the reaction mixture was slowly warmed to rt for $2 \mathrm{~h}$. After the reaction was completed, it was quenched with $1.5 \mathrm{~N} \mathrm{KHF}_{2}$ $(2 \mathrm{~mL})$ followed by removal of all the solvent under high vacuum. The residual solid was dissolved in dry acetone $(10 \mathrm{~mL})$, the insoluble salts were filtered off, and then the product was concentrated. The crude solid was purified by dissolving in a minimal amount of dry acetone and precipitating with $\mathrm{Et}_{2} \mathrm{O}$ to obtain the desired pure compound.<smiles>COc1ccccc1SCBr</smiles>

Potassium 2-Methoxy-phenylsulfanylmethyltrifluoroborate (14). The title compound was isolated as an ivory solid (94 mg, 90\% yield) starting with 2-bromoanisole. $\mathrm{mp}=154{ }^{\circ} \mathrm{C}$ (dec.). ${ }^{1} \mathrm{H}$ NMR (500 MHz, DMSO- $d_{6}$ ) $\delta 7.07$ (dd, $\left.1 \mathrm{H}, J=7.6,1.6 \mathrm{~Hz}\right), 6.95$ (td, $1 \mathrm{H}, J=7.7,1.7 \mathrm{~Hz}$ ), 6.88 $(\mathrm{td}, 1 \mathrm{H}, \mathrm{J}=7.5,1.3 \mathrm{~Hz}), 6.81(\mathrm{dd}, 1 \mathrm{H}, J=8.0,1.2 \mathrm{~Hz}), 3.74(\mathrm{~s}, 3 \mathrm{H}), 1.44$ (q, $2 \mathrm{H}, J=5.4 \mathrm{~Hz}) .{ }^{13} \mathrm{C} \mathrm{NMR}$ $\left(125.8 \mathrm{MHz}, \mathrm{DMSO}-d_{6}\right) \delta 155.5,124.3,123.6,121.3,110.1,55.8,30.8 .{ }^{19} \mathrm{~F}\left(470.8 \mathrm{MHz}\right.$, Acetone- $\left.d_{6}\right) \delta$ 141.7. ${ }^{11}$ B NMR (128.4 MHz, DMSO- $d_{6}$ ) $\delta$ 2.90. IR (neat): 3401, 2951, 2235, 1573, 1476, 1236, 1192, 1016, $982 \mathrm{~cm}^{-1}$. HRMS $(\mathrm{m} / \mathrm{z})$ : calcd. for $\mathrm{C}_{8} \mathrm{H}_{9} \mathrm{BF}_{3} \mathrm{OS} .221 .0419\left[\mathrm{M}-\mathrm{K}^{+}\right]$; found, 221.0423 .<smiles>COc1cccc(SCBr)c1</smiles>

Potassium 3-Methoxy-phenylsulfanylmethyltrifluoroborate (15). The title compound was isolated as a white solid ( $96 \mathrm{mg}, 92 \%$ yield) starting with 3-bromoanisole. $\mathrm{mp}=223{ }^{\circ} \mathrm{C}$ (dec.). ${ }^{1} \mathrm{H}$ NMR (500 MHz, Acetone- $\left.d_{6}\right) \delta 7.07$ (t, $\left.1 \mathrm{H}, J=8.3 \mathrm{~Hz}\right), 6.76(\mathrm{~m}, 2 \mathrm{H}),$,6.53 (dd, $\left.1 \mathrm{H}, J=9.1,7.2 \mathrm{~Hz}\right), 3.74$ (s, 3H), $1.80(\mathrm{~m}, 2 \mathrm{H}) .{ }^{13} \mathrm{C}$ NMR $\left(125.8 \mathrm{MHz}\right.$, Acetone- $\left.d_{6}\right) \delta 159.9,128.8,117.4,110.3,108.3,54.4,29.9 .{ }^{19} \mathrm{~F}$ $\left(470.8 \mathrm{MHz}\right.$, Acetone- $\left.d_{6}\right) \delta-141.8 .{ }^{11} \mathrm{~B}$ NMR (128.4 MHz, Acetone- $d_{6}$ ) $\delta 3.81$ (q, $\left.J=49.6 \mathrm{~Hz}\right)$. IR (neat): 3595, 3467, 2938, 2902, 1589, 1574, 1479, 1423, 1248, 1115, 1036, 969, $773 \mathrm{~cm}^{-1}$. HRMS $(\mathrm{m} / \mathrm{z})$ : calcd. for $\mathrm{C}_{8} \mathrm{H}_{9} \mathrm{BF}_{3} \mathrm{OS} .221 .0419\left[\mathrm{M}-\mathrm{K}^{+}\right]$; found, 221.0422.<smiles>BrCCSc1ccc(Br)cc1</smiles>

Potassium 4-Bromo-phenylsulfanylmethyltrifluoroborate (16). The title compound was isolated as a white solid (116 mg, 94\% yield) starting with 1,4-dibromoanisole. $\mathrm{mp}>250{ }^{\circ} \mathrm{C}$. 
${ }^{1} \mathrm{H}$ NMR $\left(500 \mathrm{MHz}\right.$, Acetone- $\left.d_{6}\right) \delta 7.32(\mathrm{~d}, 2 \mathrm{H}, J=8.8 \mathrm{~Hz}), 7.13(\mathrm{~d}, 2 \mathrm{H}, J=8.7 \mathrm{~Hz}), 1.78(\mathrm{~m}, 2 \mathrm{H}) .{ }^{13} \mathrm{C}$ NMR $\left(125.8 \mathrm{MHz}\right.$, Acetone- $\left.d_{6}\right) \delta 133.7,130.9,126.8,115.2 .{ }^{19} \mathrm{~F}\left(470.8 \mathrm{MHz}\right.$, Acetone- $\left.d_{6}\right) \delta-140.5 .{ }^{11} \mathrm{~B} \mathrm{NMR}$ (128.4 MHz, Acetone- $\left.d_{6}\right) \delta 3.48$ (q, $J=51.4 \mathrm{~Hz}$ ). IR (neat): 2899, 1474, 1385, 1212, 1068, 978, 949, $809 \mathrm{~cm}^{-}$

${ }^{1}$. HRMS $(\mathrm{m} / \mathrm{z})$ : calcd. for $\mathrm{C}_{7} \mathrm{H}_{6} \mathrm{BBrF}_{3} \mathrm{~S} .268 .9419\left[\mathrm{M}-\mathrm{K}^{+}\right]$; found, 268.9431.

\section{Reaction Condition for Preparing Compounds 17 and 18.}

$\mathrm{Br}_{\mathrm{BF}_{3} \mathrm{~K}}$ Potassium 5-Bromopentyltrifluoroborate (17). ${ }^{3}$ To a soln of 5-bromo-1-pentene $(1.0 \mathrm{~mL}, 10 \mathrm{mmol})$ in $\mathrm{CH}_{2} \mathrm{Cl}_{2}(5 \mathrm{~mL})$ in the presence of $1 \mathrm{~mol} \% \mathrm{Rh}\left(\mathrm{PPh}_{3}\right)_{3} \mathrm{Cl}$ catalyst was added pinacolborane (1.0 M THF solution, $10 \mathrm{~mL}, 10 \mathrm{mmol})$ at $0{ }^{\circ} \mathrm{C}$. The reaction mixture was stirred for $2 \mathrm{~h}$ at $\mathrm{rt}$, and then extracted with $\mathrm{Et}_{2} \mathrm{O}(80 \mathrm{~mL})$, washed with $\mathrm{H}_{2} \mathrm{O}(30 \mathrm{~mL})$, dried $\left(\mathrm{Mg}_{2} \mathrm{SO}_{4}\right)$, and filtered. After the removal of solvent, the crude product was obtained. The crude compound was purified by column chromatography on silica gel with eluting with hexane/ether (20:1 to 10:1, v/v) to obtain the hydroborated intermediate ( $1.33 \mathrm{~g}, 48 \%$ yield). A part $(500 \mathrm{mg}, 1.8 \mathrm{mmol})$ of the hydroborated intermediate was dissolved in $\mathrm{Et}_{2} \mathrm{O}(5 \mathrm{~mL})$. To the solution was added $\mathrm{KHF}_{2}(0.84 \mathrm{~g}, 11 \mathrm{mmol})$ at $0{ }^{\circ} \mathrm{C}$ followed by the addition of $\mathrm{H}_{2} \mathrm{O}$ $(2 \mathrm{~mL})$. After the mixture was stirred for $1 \mathrm{~h}$, acetone $(50 \mathrm{~mL})$ was added and the suspension was concentrated on a rotary evaporator. The resulting white solids were subjected to high vacuum for $2 \mathrm{~h}$. The crude compound was purified by dissolving in acetone and precipitating with $\mathrm{Et}_{2} \mathrm{O}$ to obtain the desired compound 17 as a white solid (253 mg, 55\% yield). mp > 250 ${ }^{\circ} \mathrm{C} .{ }^{1} \mathrm{H}$ NMR (500 MHz, DMSO- $\left.d_{6}\right) \delta 3.46(\mathrm{t}$, $2 \mathrm{H}, J=6.9 \mathrm{~Hz}), 1.73(\mathrm{~m}, 2 \mathrm{H}), 1.27(\mathrm{~m}, 2 \mathrm{H}), 1.13(\mathrm{~m}, 2 \mathrm{H}),-0.07\left(\mathrm{~m}, 2 \mathrm{H},-\mathrm{CH}_{2}-\mathrm{B}\right) .{ }^{13} \mathrm{C} \mathrm{NMR}(125.8 \mathrm{MHz}$, DMSO- $\left.d_{6}\right) \delta 36.1,33.3,31.8,25.2 .{ }^{19} \mathrm{~F}\left(470.8 \mathrm{MHz}, \mathrm{DMSO}-d_{6}\right) \delta$-137.2. ${ }^{11} \mathrm{~B}$ NMR (128.4 MHz, DMSO- $\left.d_{6}\right)$ $\delta$ 4.58. IR (neat): 3435, 2925, 2910, 2841, 1463, 1340, 1298, 1239, 1098, 1077, 1036, 935, $862 \mathrm{~cm}^{-1}$. HRMS $(\mathrm{m} / \mathrm{z})$ : calcd. for $\mathrm{C}_{5} \mathrm{H}_{10} \mathrm{BBrF}_{3}, 217.0011\left[\mathrm{M}-\mathrm{K}^{+}\right]$; found, 217.0003.

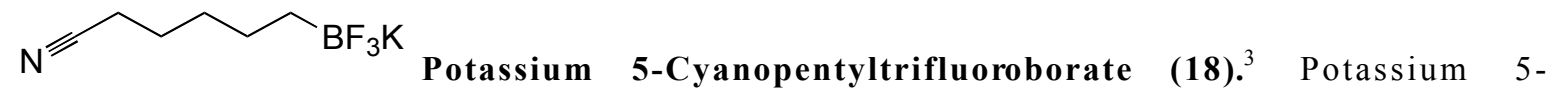
bromopentyltrifluoroborate $17^{3}$ (51.4 $\left.\mathrm{mg}, 0.2 \mathrm{mmol}\right)$ and $\mathrm{KCN}$ (13 $\left.\mathrm{mg}, 0.20 \mathrm{mmol}\right)$ were dissolved in DMSO $(1.0 \mathrm{~mL})$. The reaction mixture was heated at $80{ }^{\circ} \mathrm{C}$ for $1 \mathrm{~h}$ and concentrated under high vacuum. The residual product was dissolved in dry acetone $(2.0 \mathrm{~mL})$, the insoluble salts were filtered off, and then the product was concentrated. The crude solid was purified by dissolving in a minimal amount of dry acetone and precipitating with $\mathrm{Et}_{2} \mathrm{O}$ to obtain the desired pure compound $\mathbf{1 8}$ as a white solid $(37.4 \mathrm{mg}, 92 \%$ yield $) . \mathrm{mp}=177-180{ }^{\circ} \mathrm{C}$. ${ }^{1} \mathrm{H}$ NMR (360.13 MHz, DMSO- $d_{6}$ ) $\delta 2.39$ (t, $\left.2 \mathrm{H}, J=7.1 \mathrm{~Hz}\right), 1.45$ (q, 2H, $\left.J=7.3 \mathrm{~Hz}\right), 1.24(\mathrm{~m}, 2 \mathrm{H}), 1.11$ (m, 2H), -0.07 (m, 2H, - $\mathrm{CH}_{2}$-B). ${ }^{13} \mathrm{C}$ NMR (90.6 MHz, DMSO- $\left.d_{6}\right) \delta 121.3,32.3,25.5,25.2,25.1,16.6 .{ }^{19} \mathrm{~F}(470.8$ MHz, DMSO- $\left.d_{6}\right) \delta-137.2 .{ }^{11} \mathrm{~B}$ NMR (128.4 MHz, DMSO- $d_{6}$ ) $\delta$ 4.41. IR (neat): 2937, 2907, 2843, 2260, 
$1459,1420,1057,961,870 \mathrm{~cm}^{-1}$. HRMS $(\mathrm{m} / \mathrm{z})$ : calcd. for $\mathrm{C}_{6} \mathrm{H}_{10} \mathrm{BF}_{3} \mathrm{~N} .164 .0858\left[\mathrm{M}-\mathrm{K}^{+}\right]$; found, 164.0856 .

\section{References}

(1) Molander, G. A.; Ribagorda, M. J. Am. Chem. Soc. 2003, 125, 11148.

(2) Molander, G. A.; Figueroa, R. Org. Lett. 2006, 8, 75.

(3) Molander, G. A.; Ito, T. Org. Lett. 2001, 3, 393. 
镸

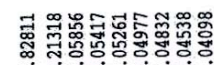

ivivivi

$\mathrm{Br} \frown \mathrm{BF}_{3} \mathrm{~K}$

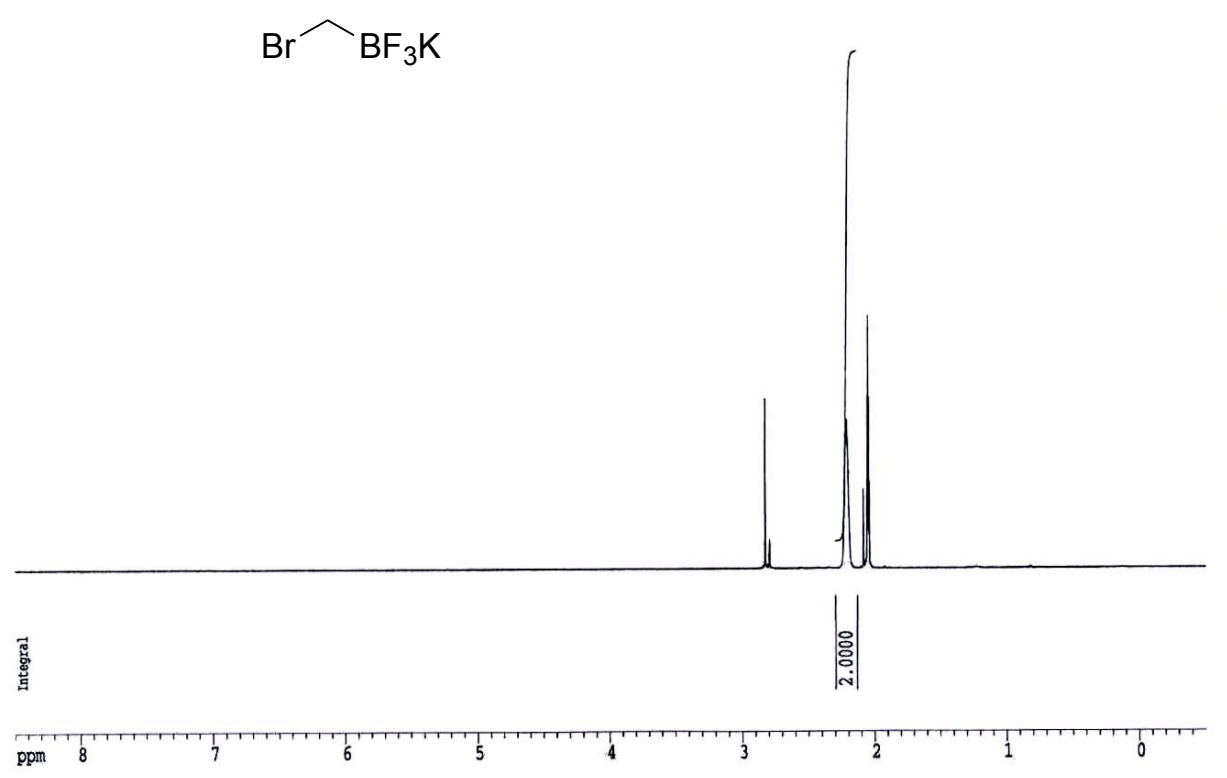

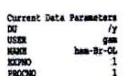

n:

in

sin

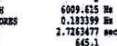

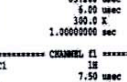

等

登

等

镸

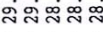

$\psi$

$\mathrm{Br} \frown \mathrm{BF}_{3} \mathrm{~K}$
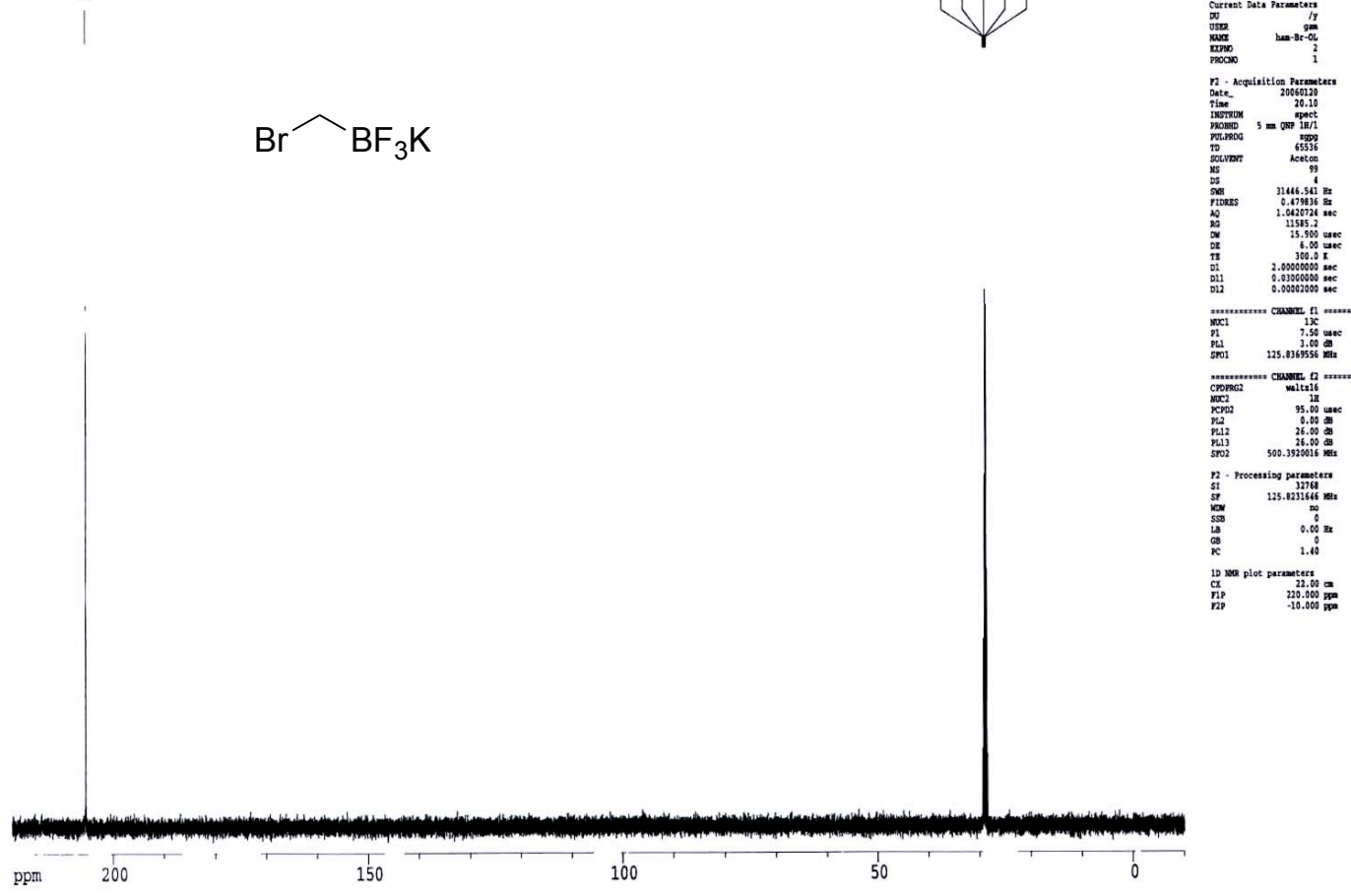
$\mathrm{Br} \widehat{B F}_{3} \mathrm{~K}$
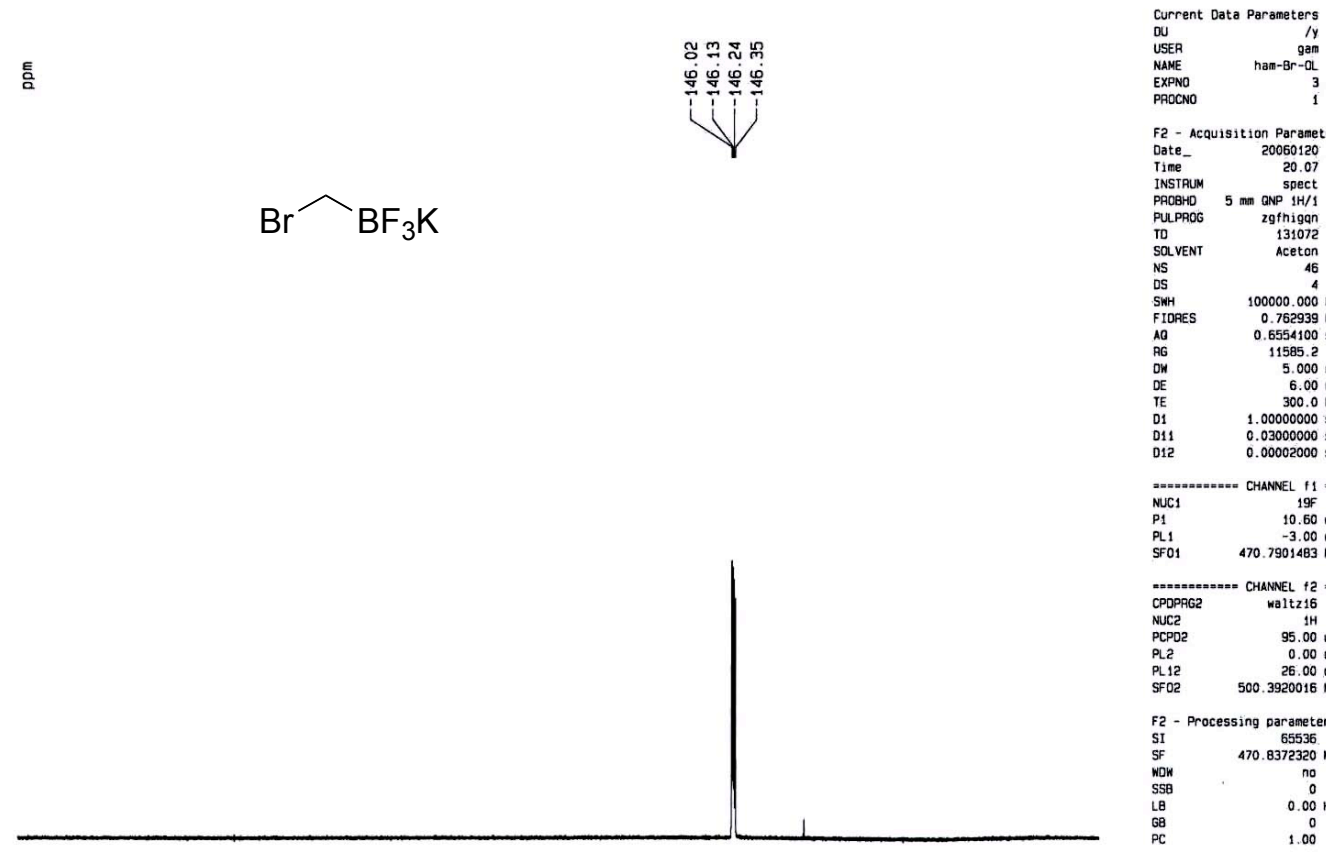

F2 - Acquisition Parameters

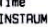

$5 \mathrm{~mm} \mathrm{GNP} \mathrm{spcct}$

$\begin{array}{lr}\text { PULPROG } & \text { 29thigan } \\ \text { TD } & 131072\end{array}$

$\begin{array}{lr}\text { SQVENT } & \text { Aceton } \\ \text { NS } & 46 \\ \text { DS } & 4\end{array}$

$100000.000 \mathrm{H}$
0.762939

$\begin{array}{ll}A 0 & 0.6554100 \mathrm{se} \\ \text { AG } & 11585.2\end{array}$

1585.2
5.000 usec

$\begin{array}{cc}\text { TE } & 6.00 \text { use } \\ \text { TE } & 30.0 \mathrm{~K} \\ \text { D1 } & 1.00000000 \mathrm{se}\end{array}$

$\begin{array}{ll}011 & 0.03000000 \mathrm{sec} \\ 012 & 0.0000200 \mathrm{sec}\end{array}$

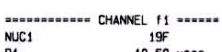

$\begin{array}{lr}\text { P1 } & 10.60 \text { usec } \\ \text { PL1 } & -3.0008 \\ \text { SFO1 } & 470.7901483 \text { MHZ }\end{array}$

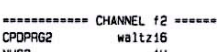

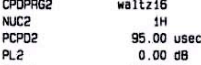

$\begin{array}{lr}\text { PL2 } & 0.000 \mathrm{~dB} \\ \text { PL12 } & 26.00 \mathrm{oB} \\ \text { SFO2 } & 500.3920015 \mathrm{MHz}\end{array}$

F2 - Processing Darameters

$\begin{array}{ll}\text { SI } & 65536 . \\ \text { SF } & 470.8372320\end{array}$

$\begin{array}{ll}\text { NOW } & 00 \\ \text { SSB } & 0 \\ \text { LB } & 0.00 \mathrm{~Hz} \\ 68 & 0 \\ \text { PC } & 1.00\end{array}$
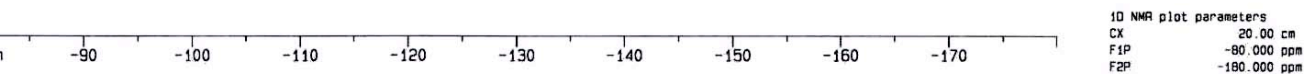

區

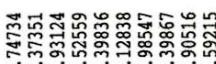

mon

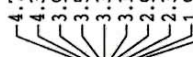

$\mathrm{Br} \curvearrowright \mathrm{BF}_{3} \mathrm{~K}$
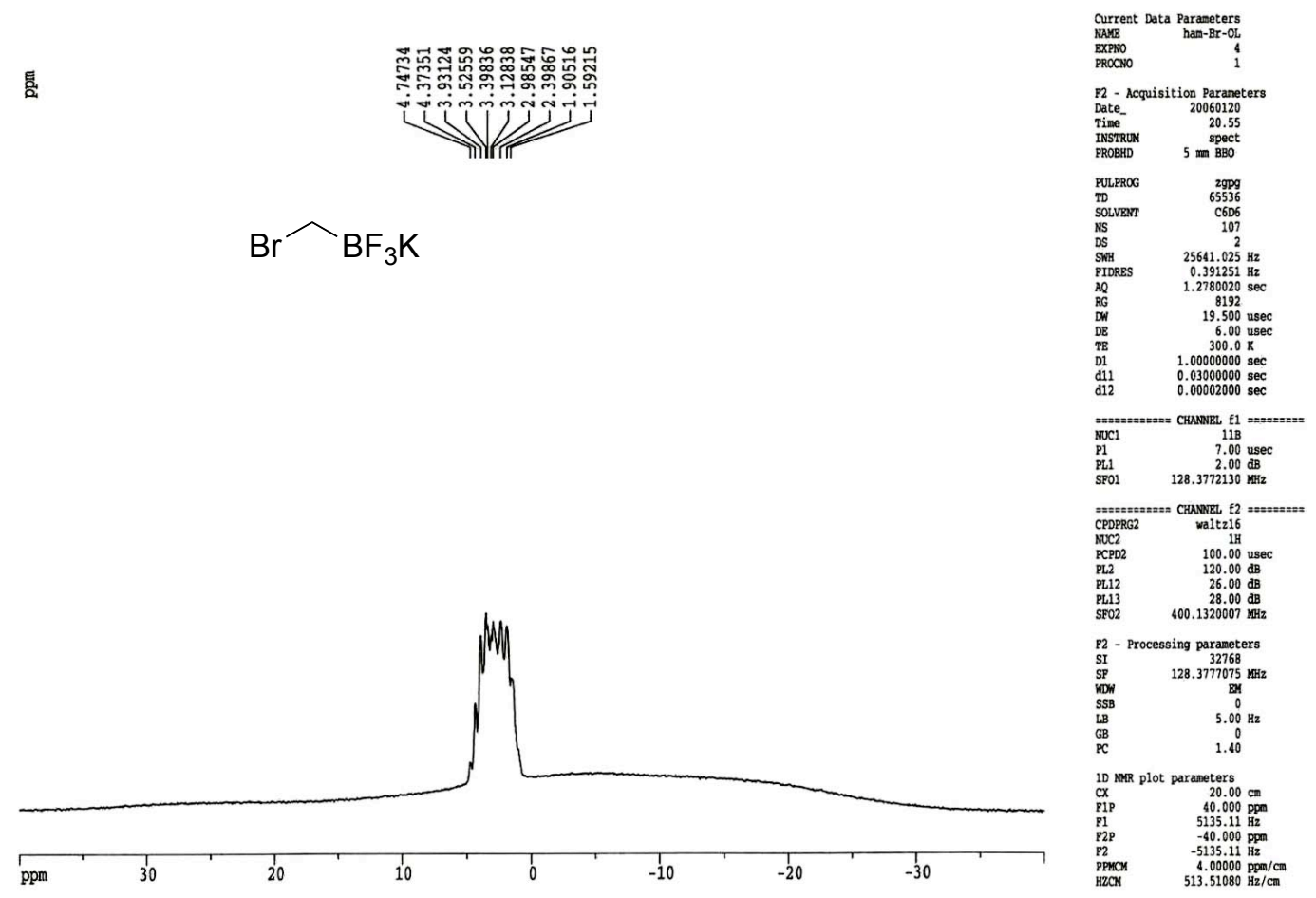


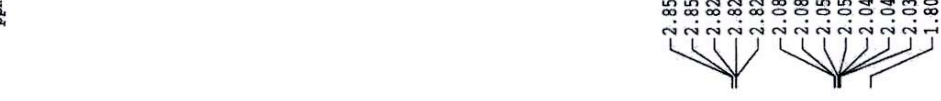
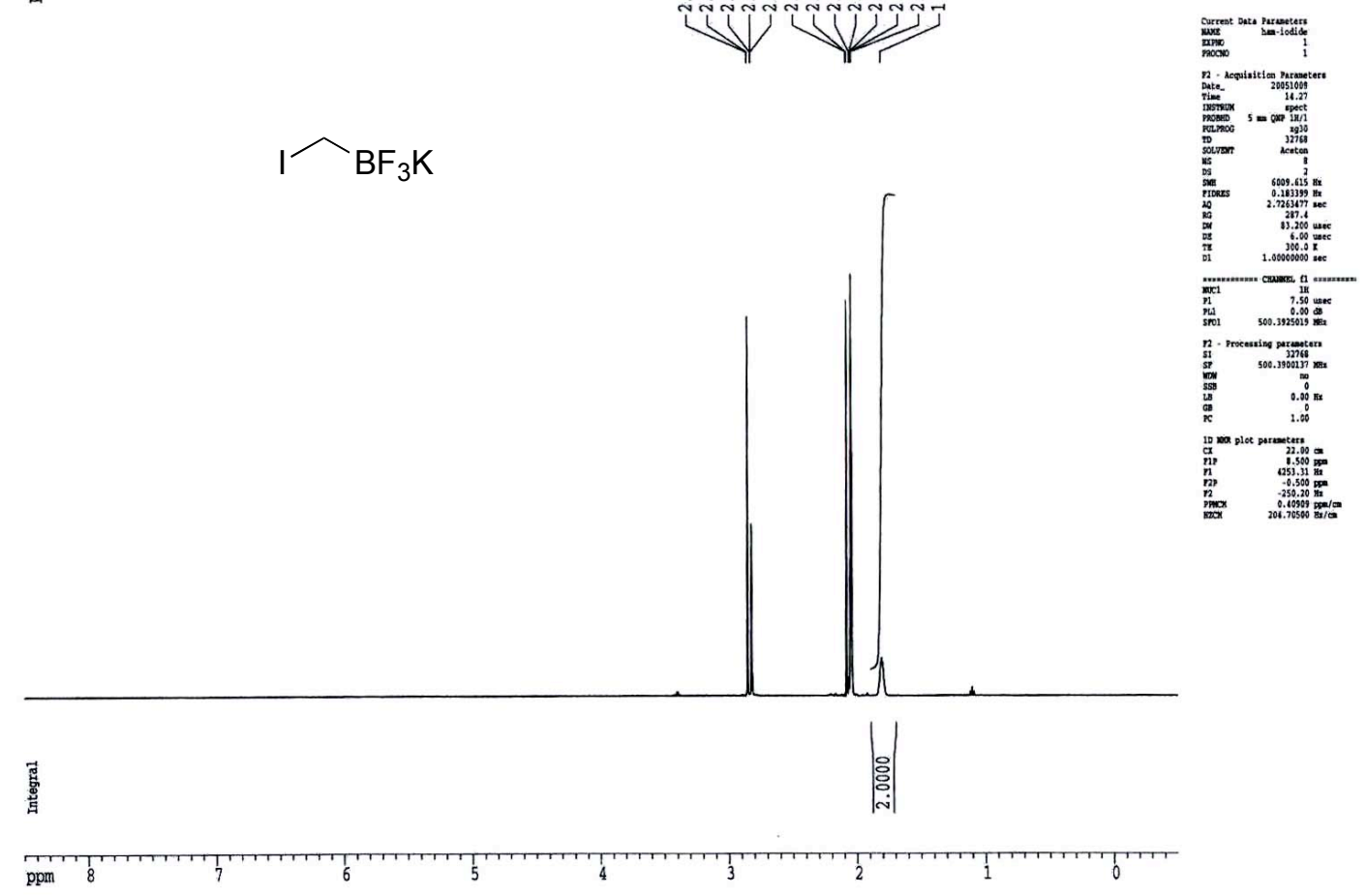

镸

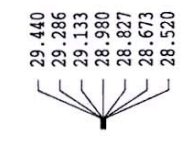

$\widehat{B F}_{3} \mathrm{~K}$
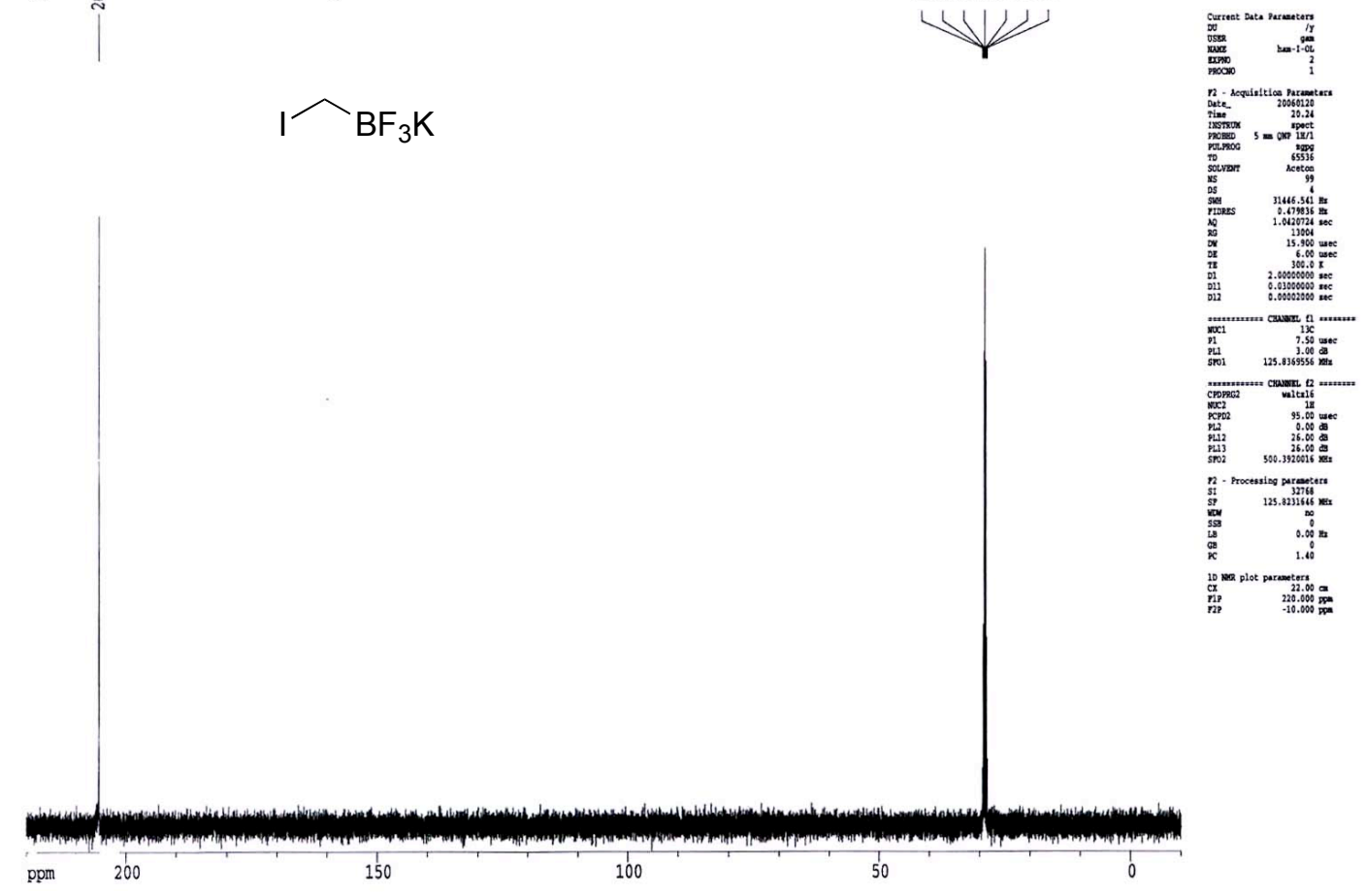

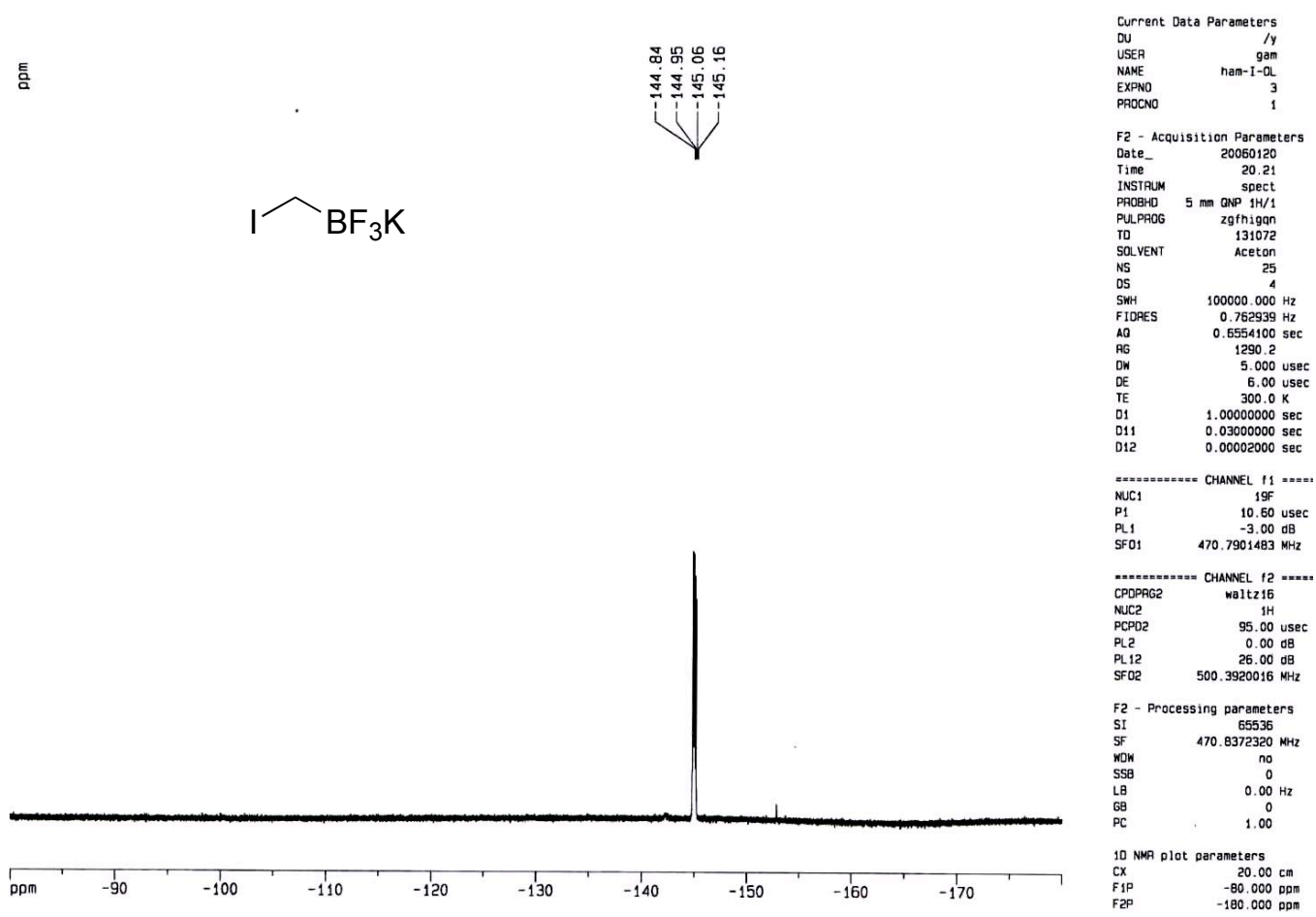

镸
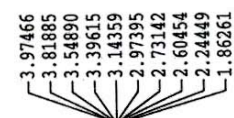

$\widehat{B F}_{3} \mathrm{~K}$
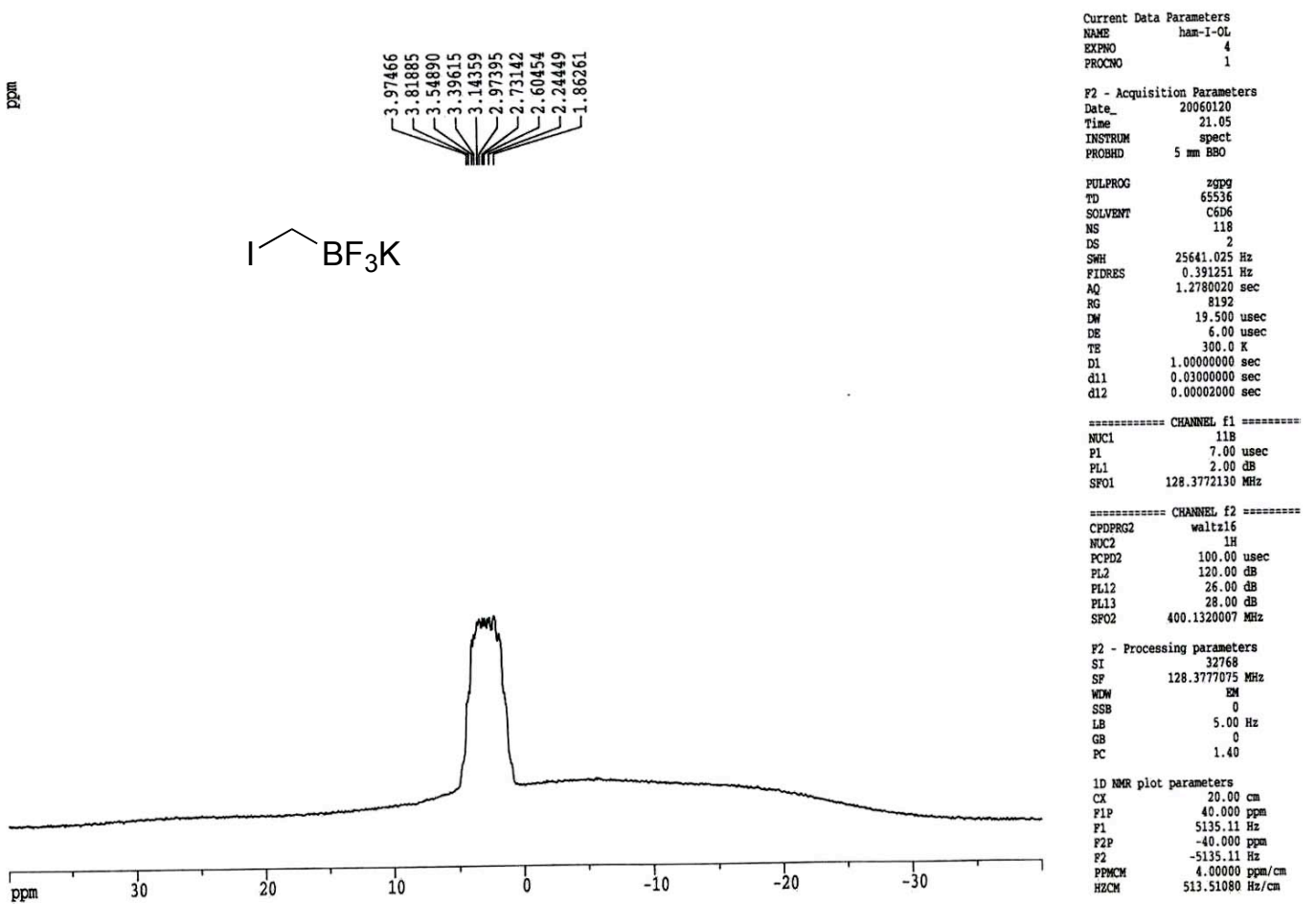

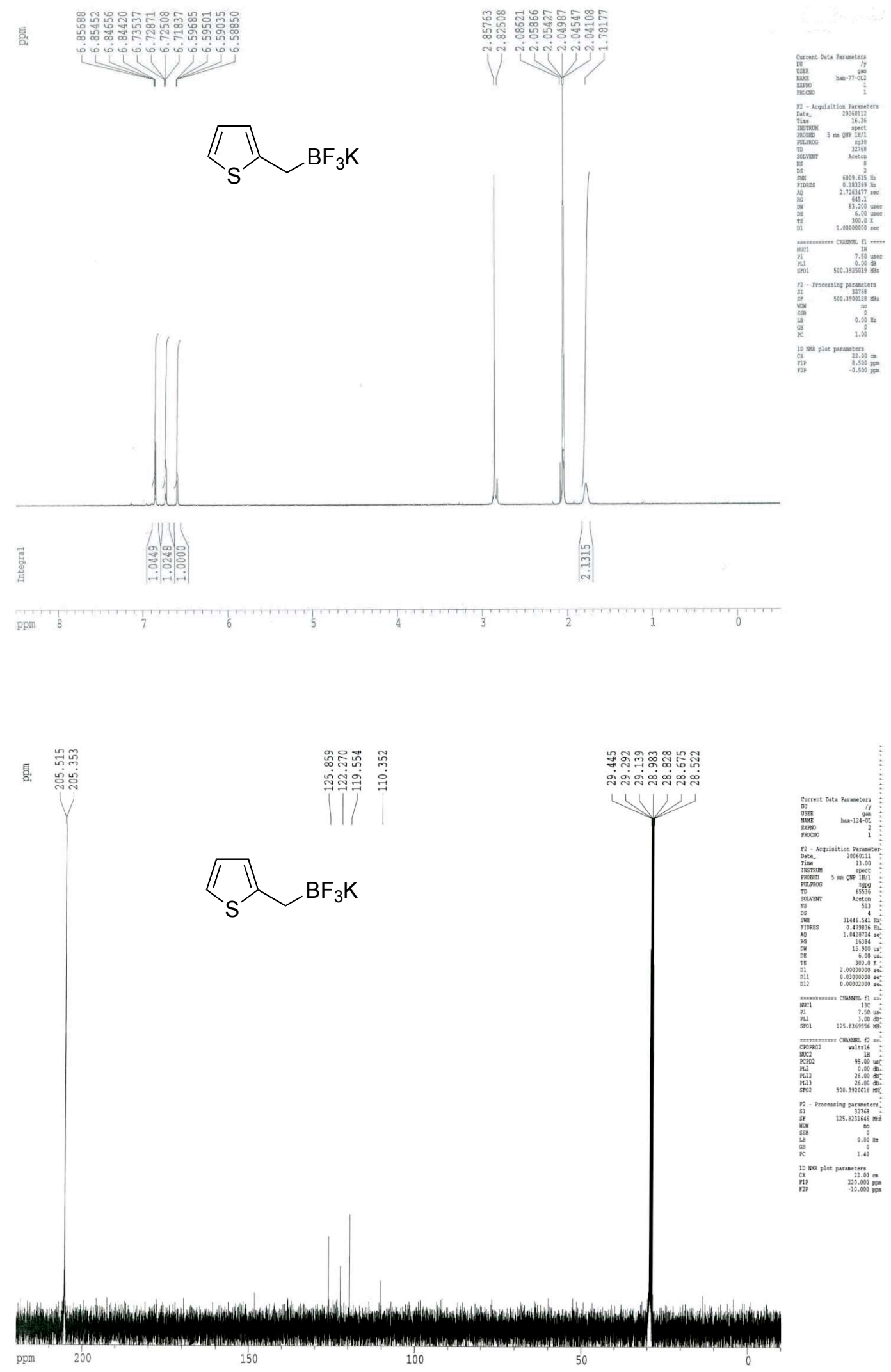

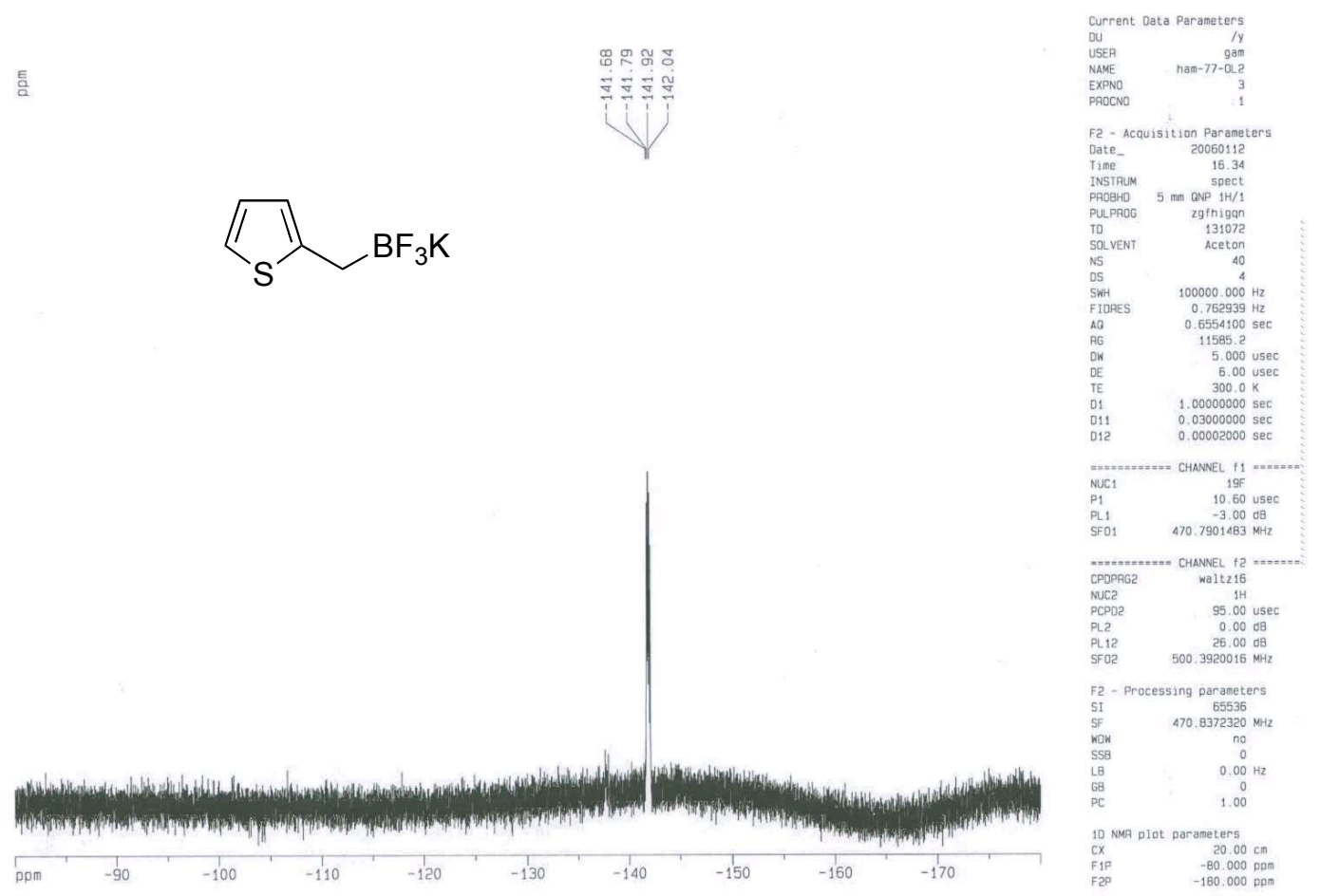

镸
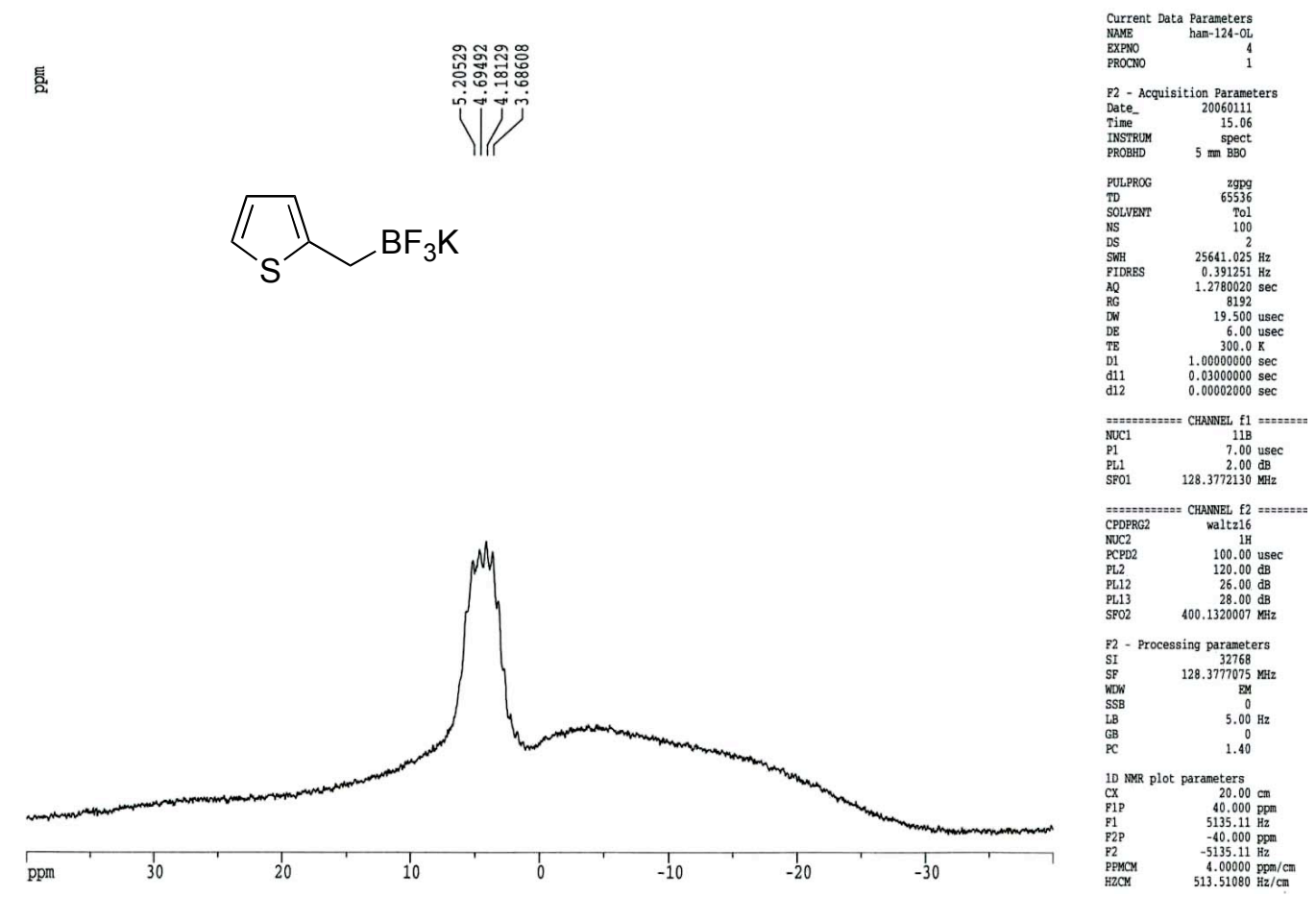


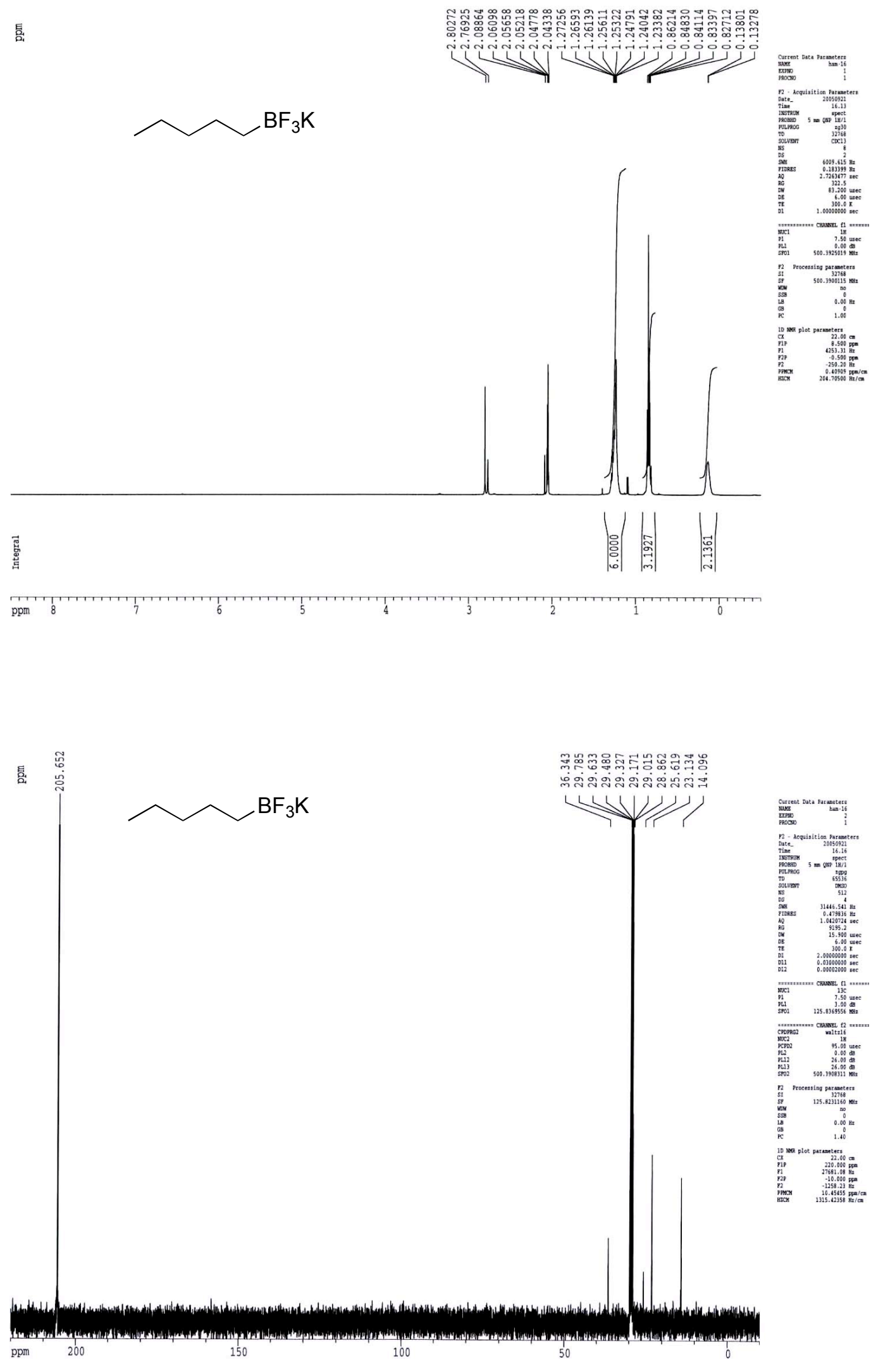



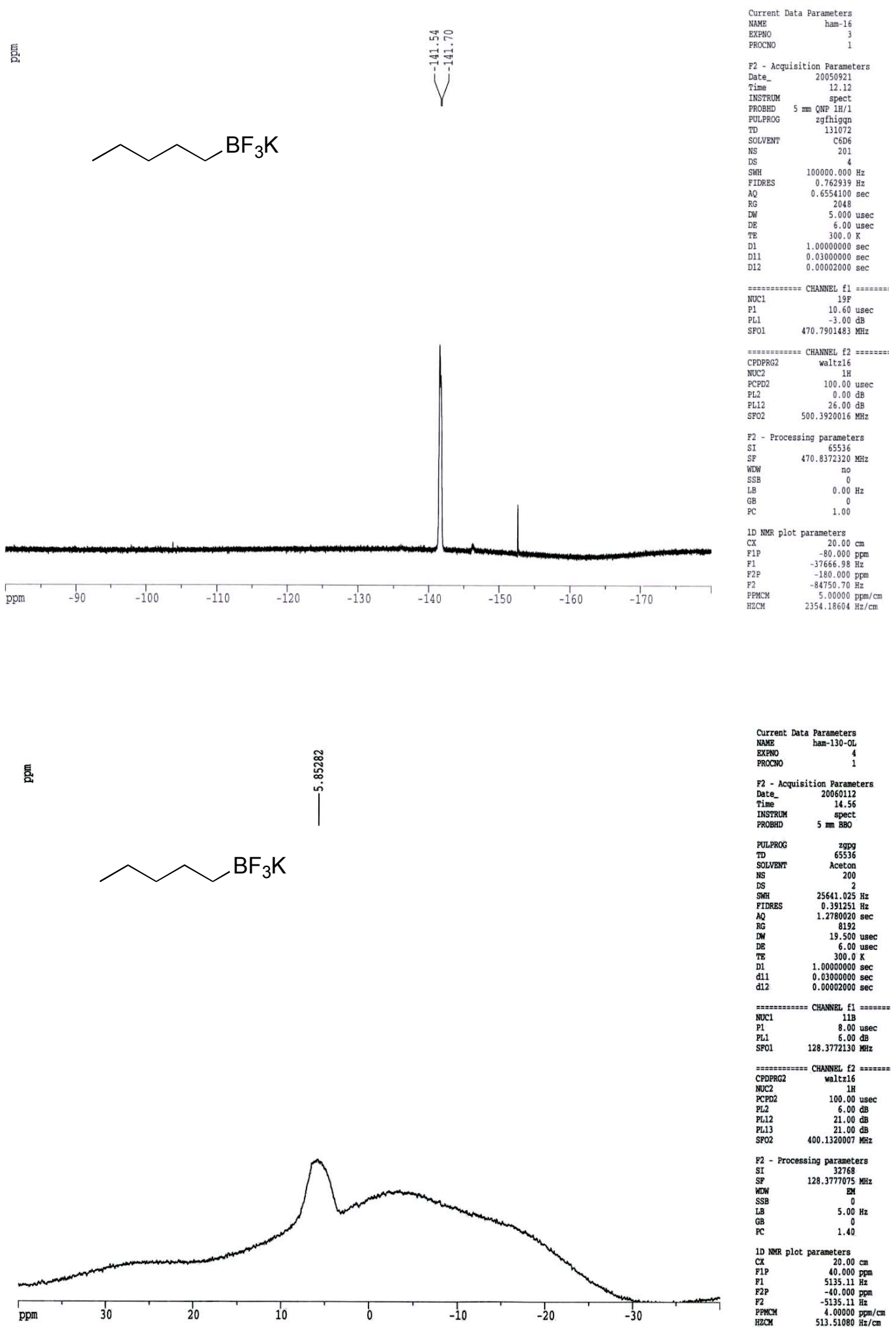

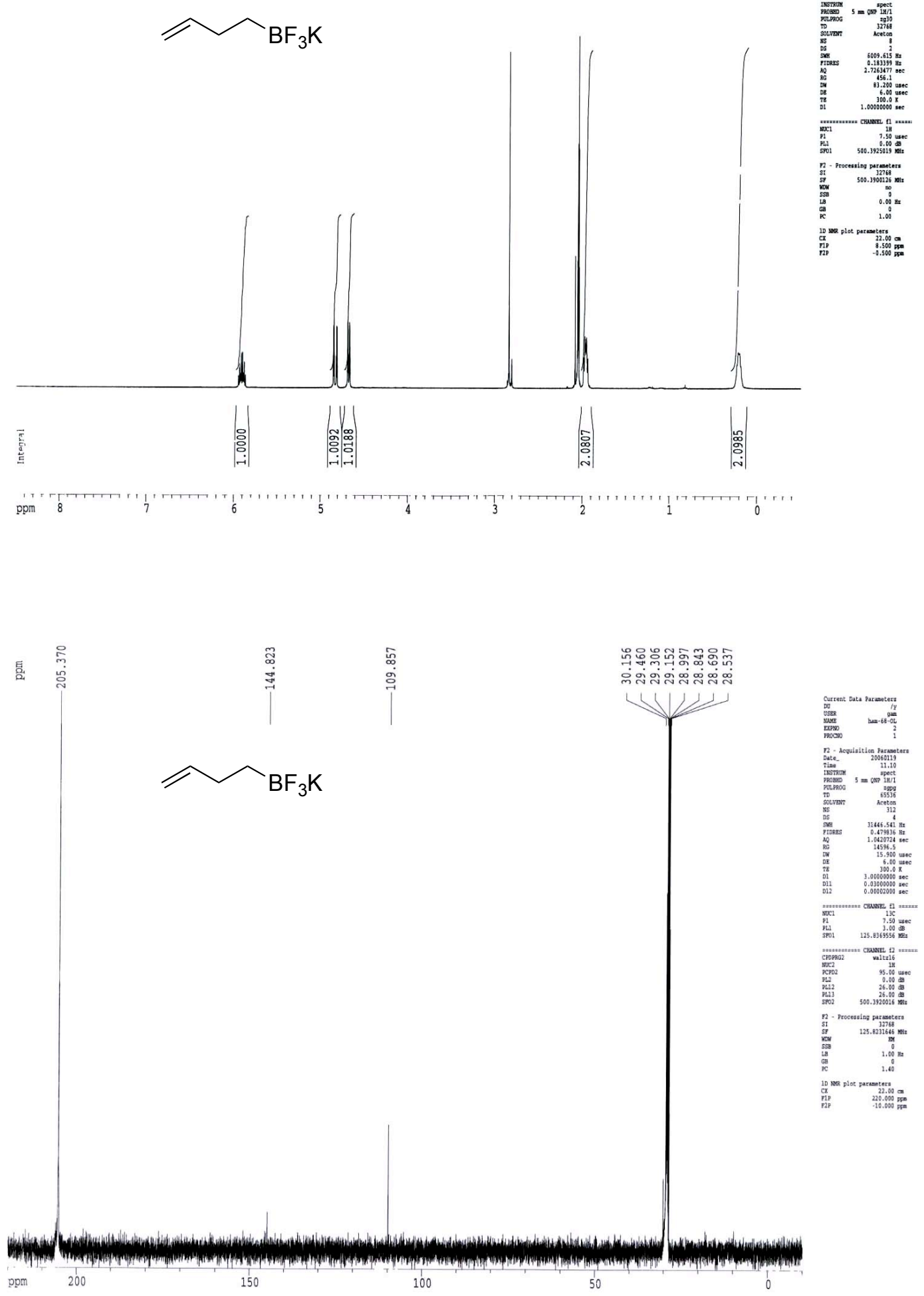

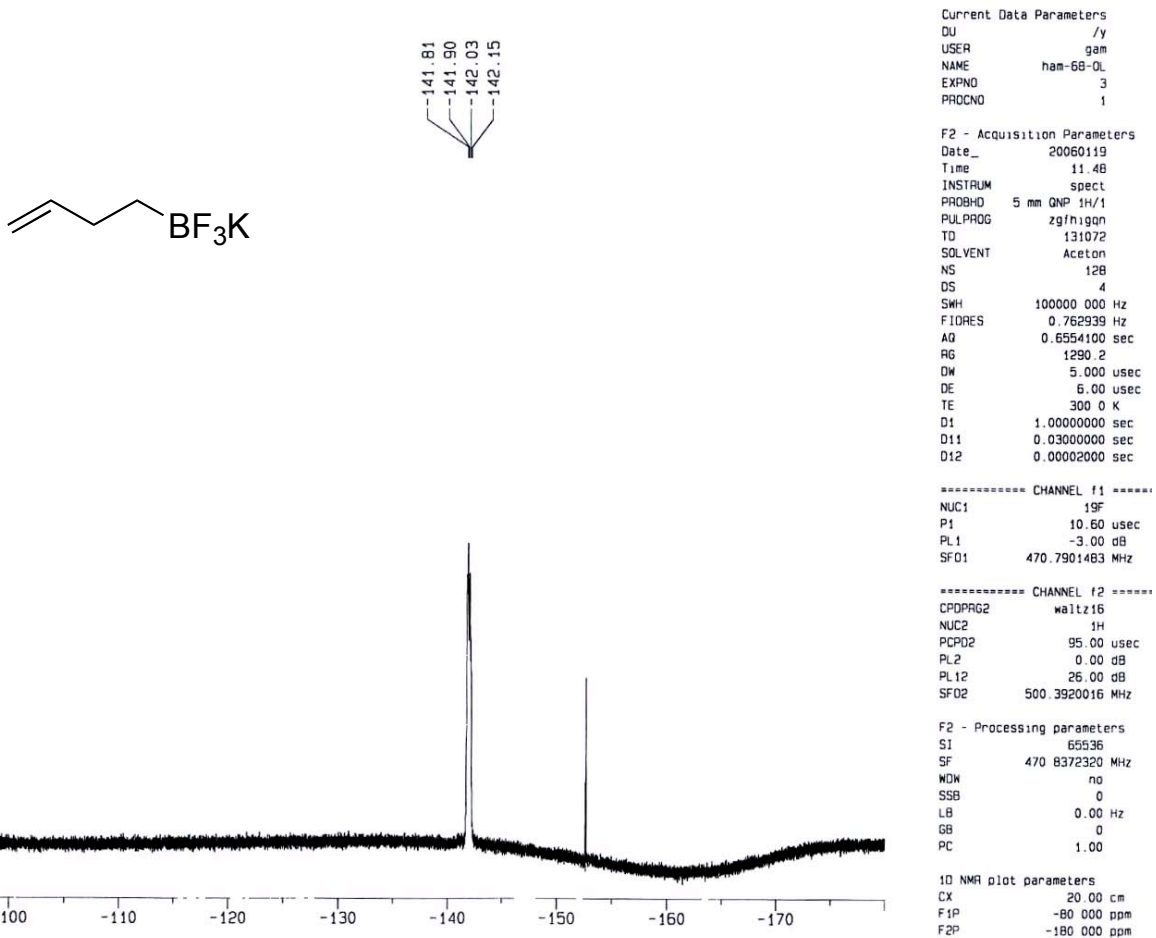

晋
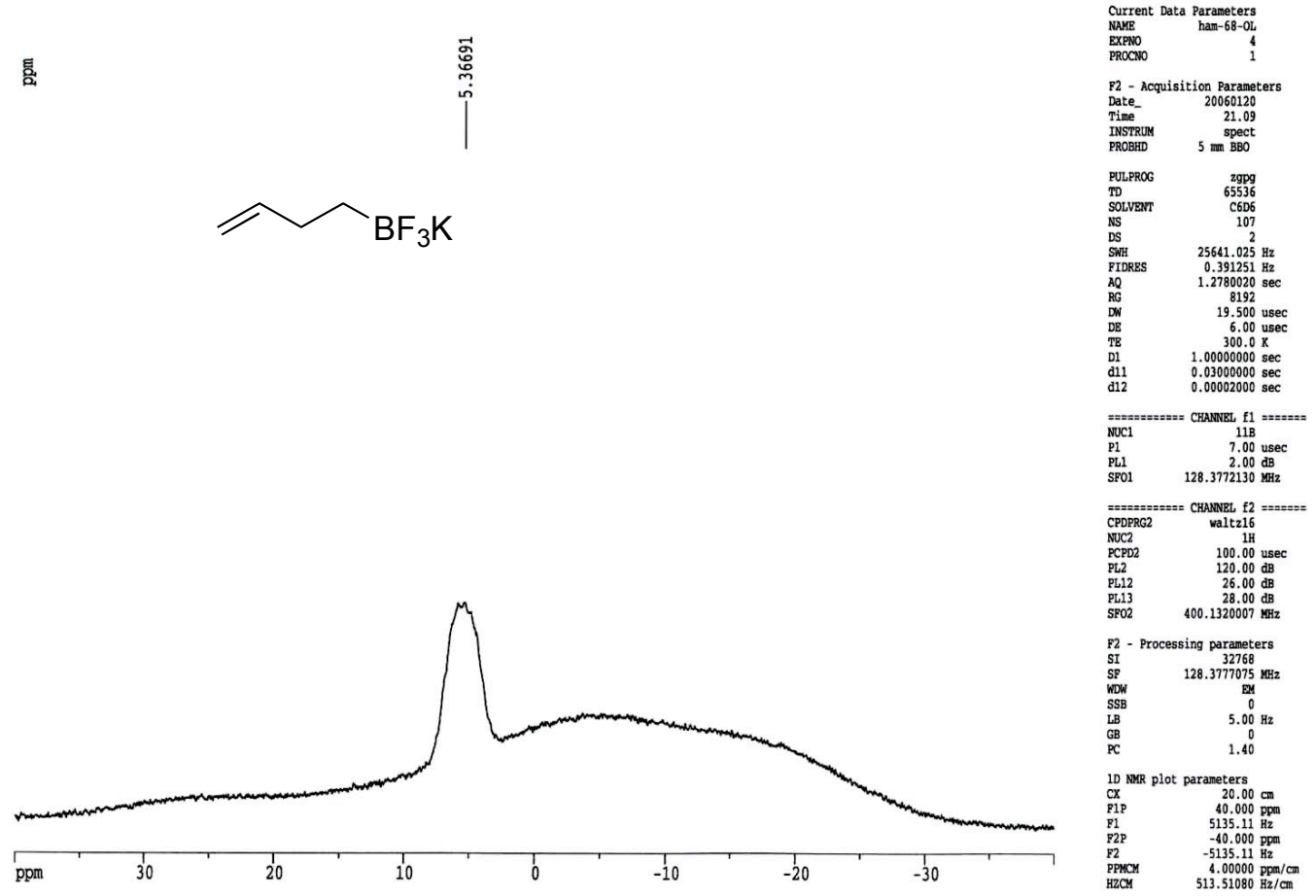

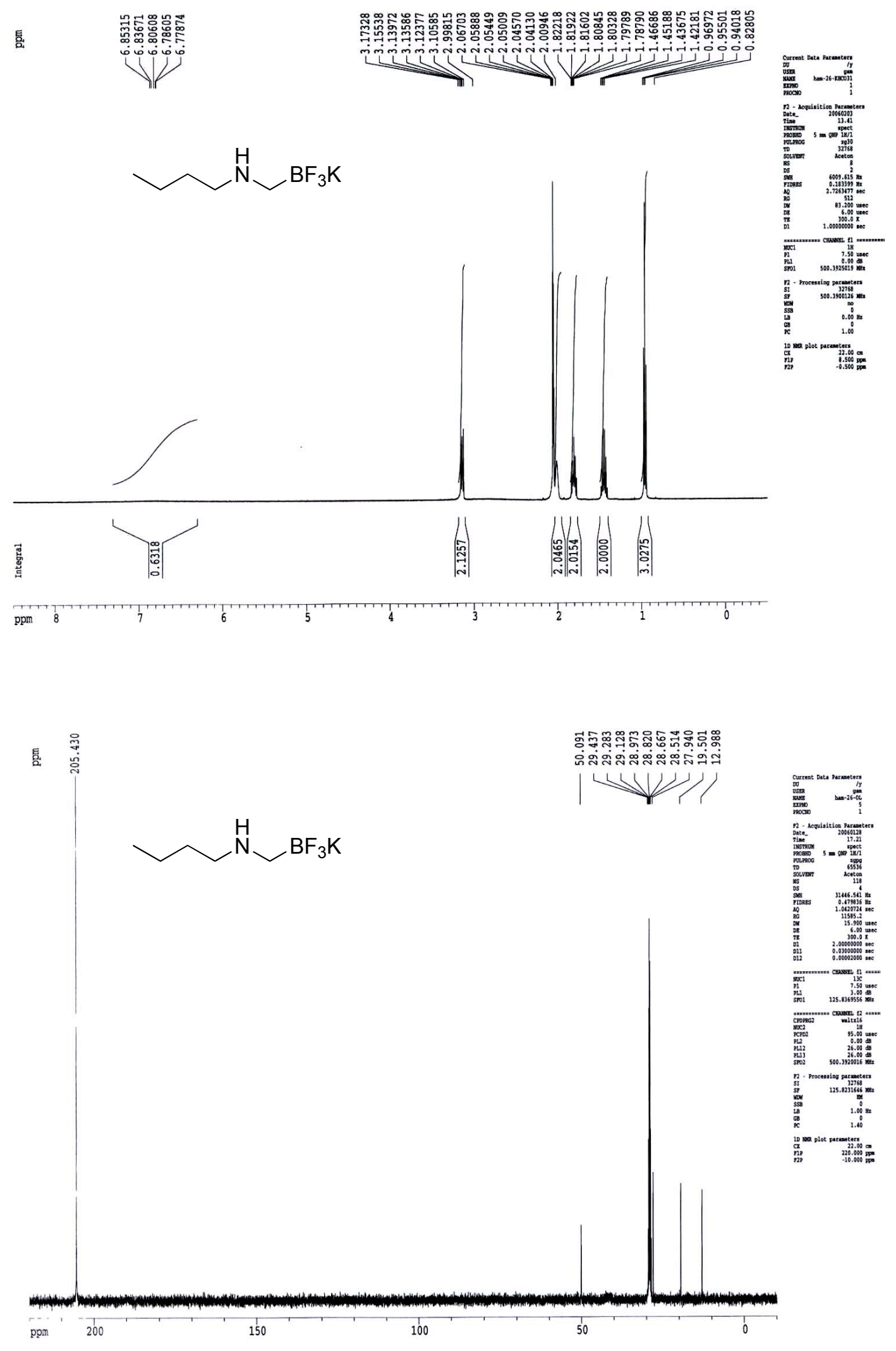

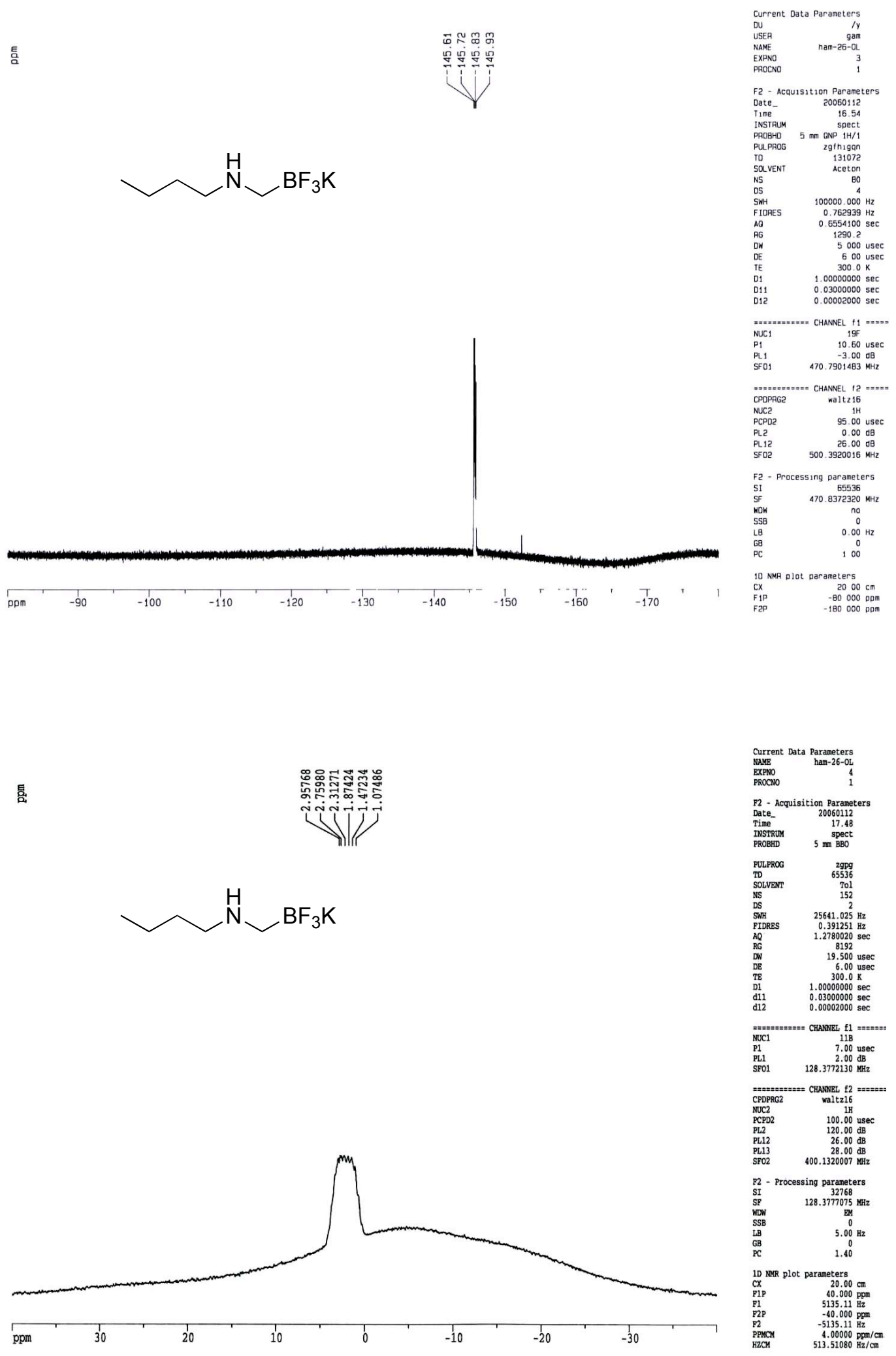

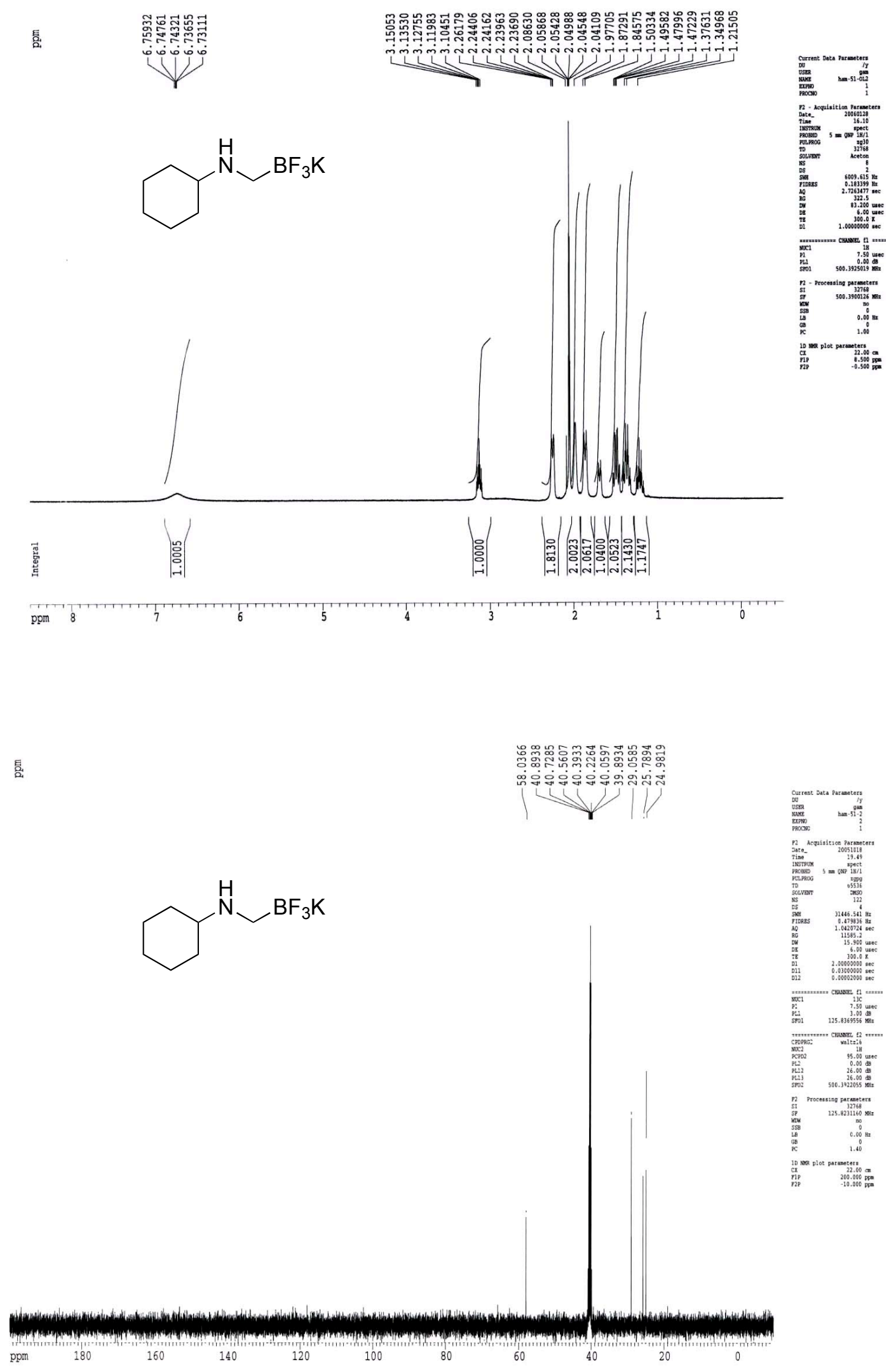

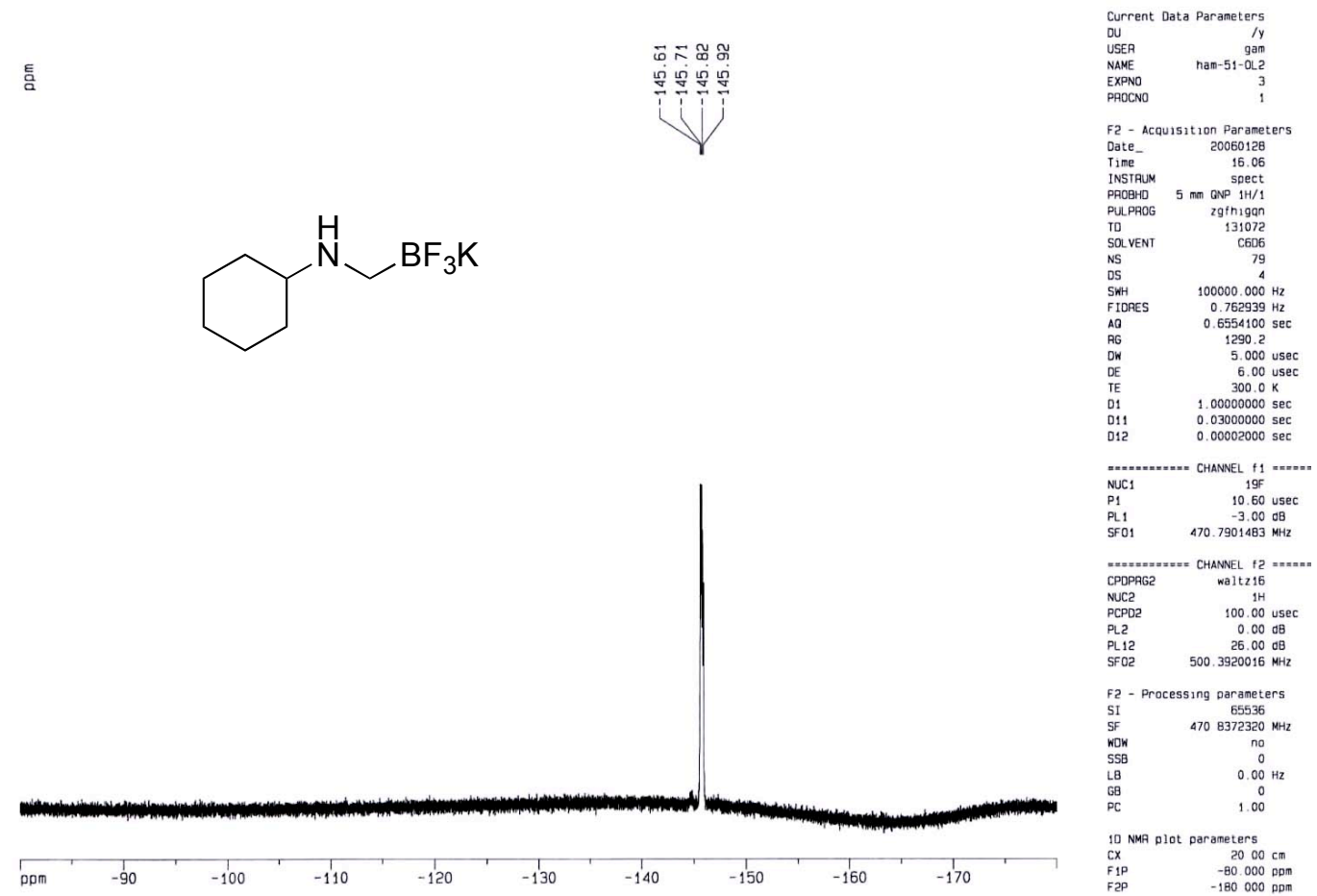

镸
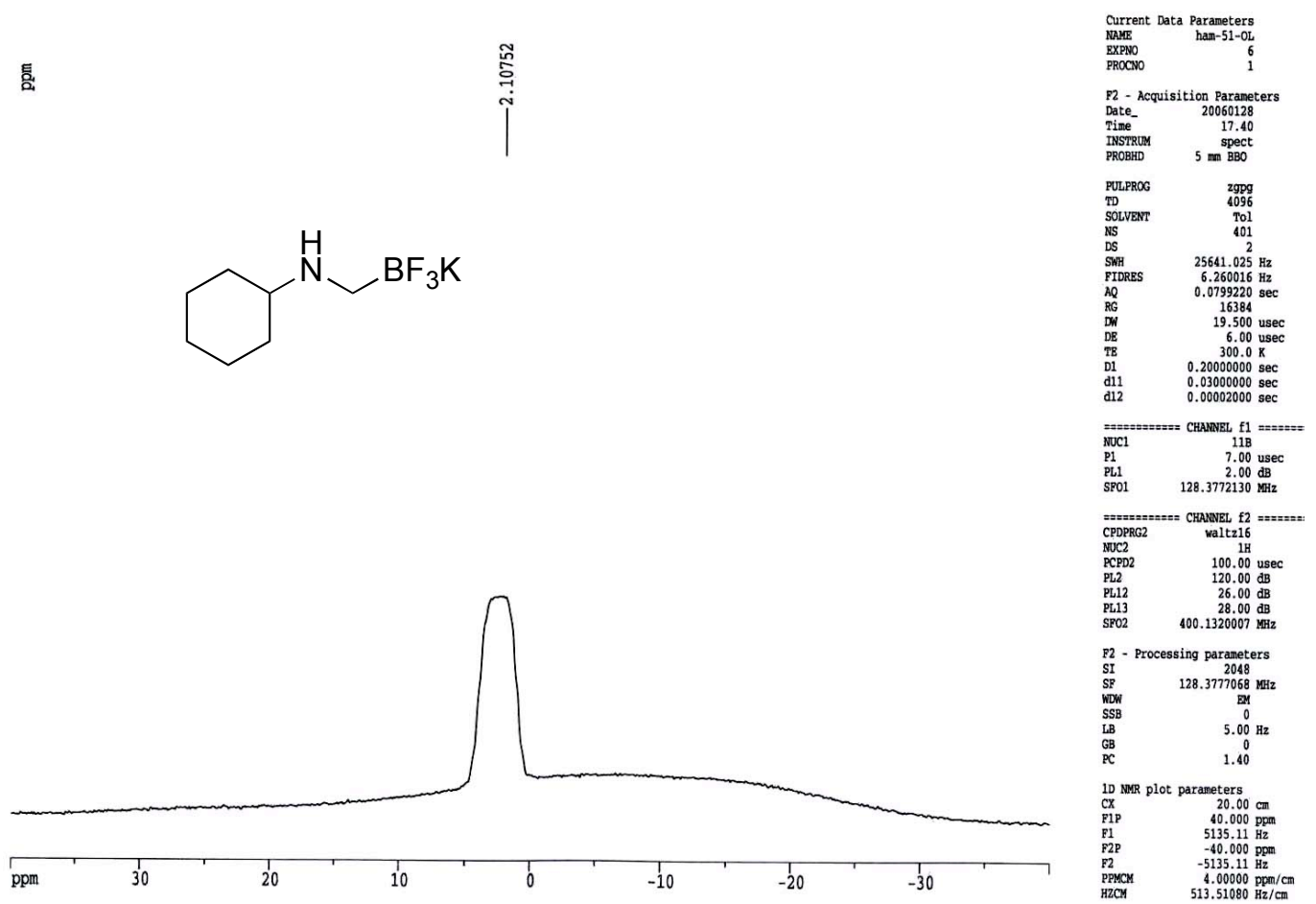

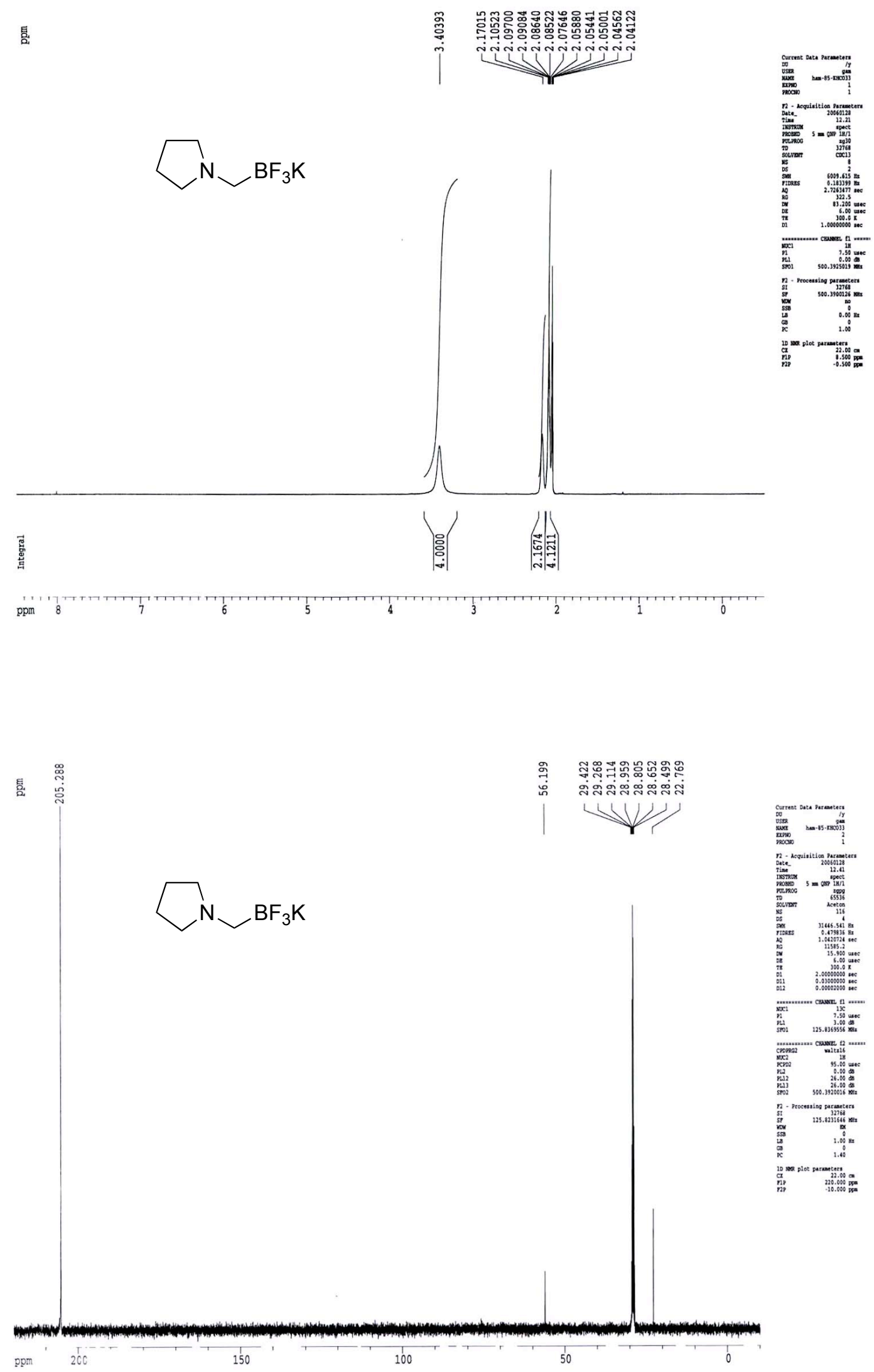

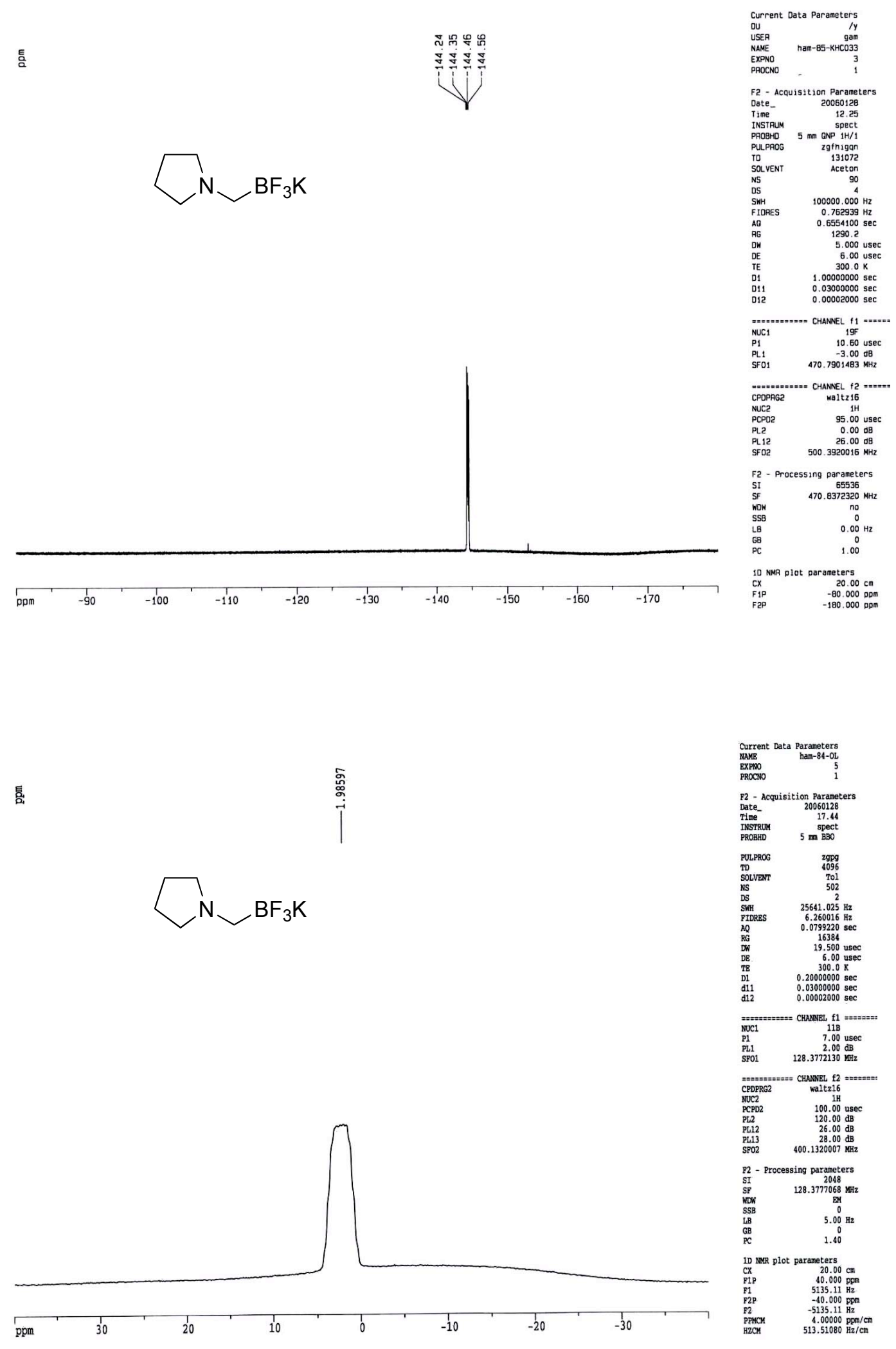

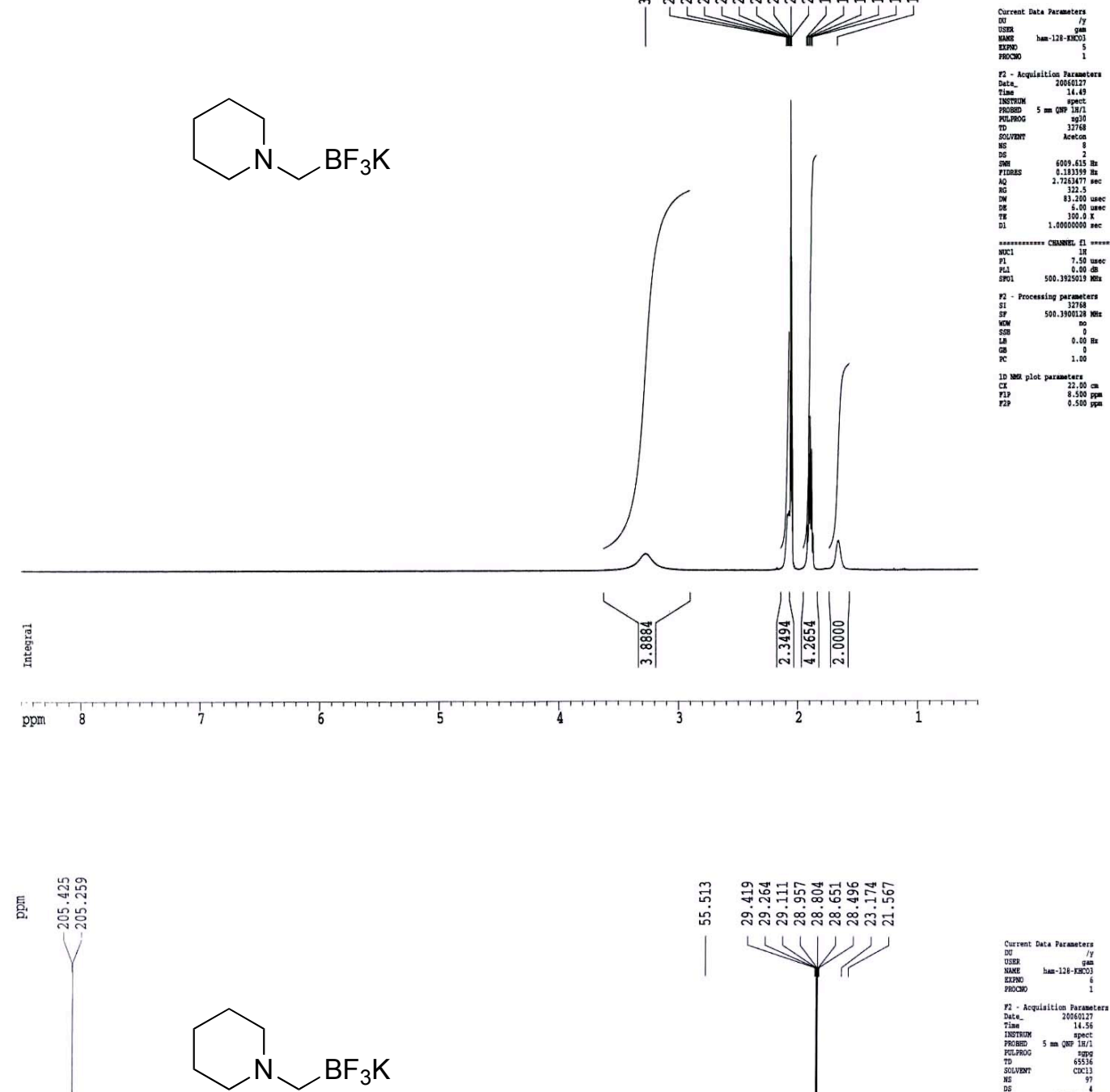

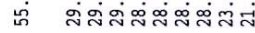

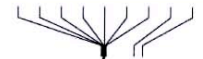

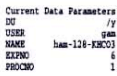

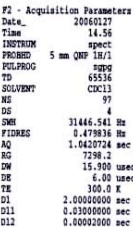

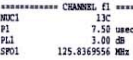

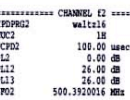

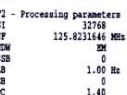

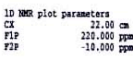

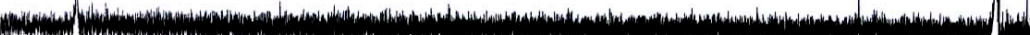

op:n 200

150

100

50 

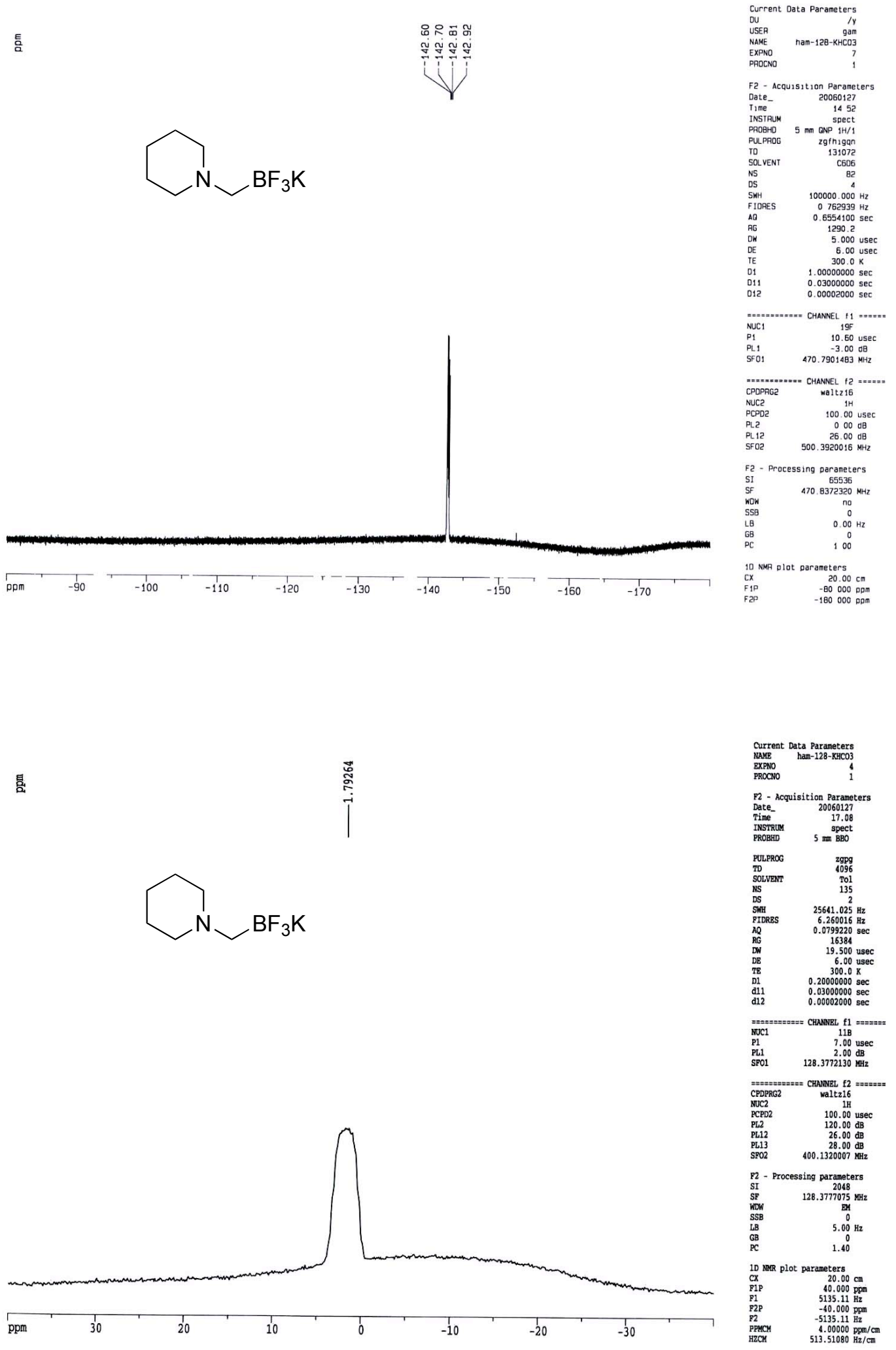

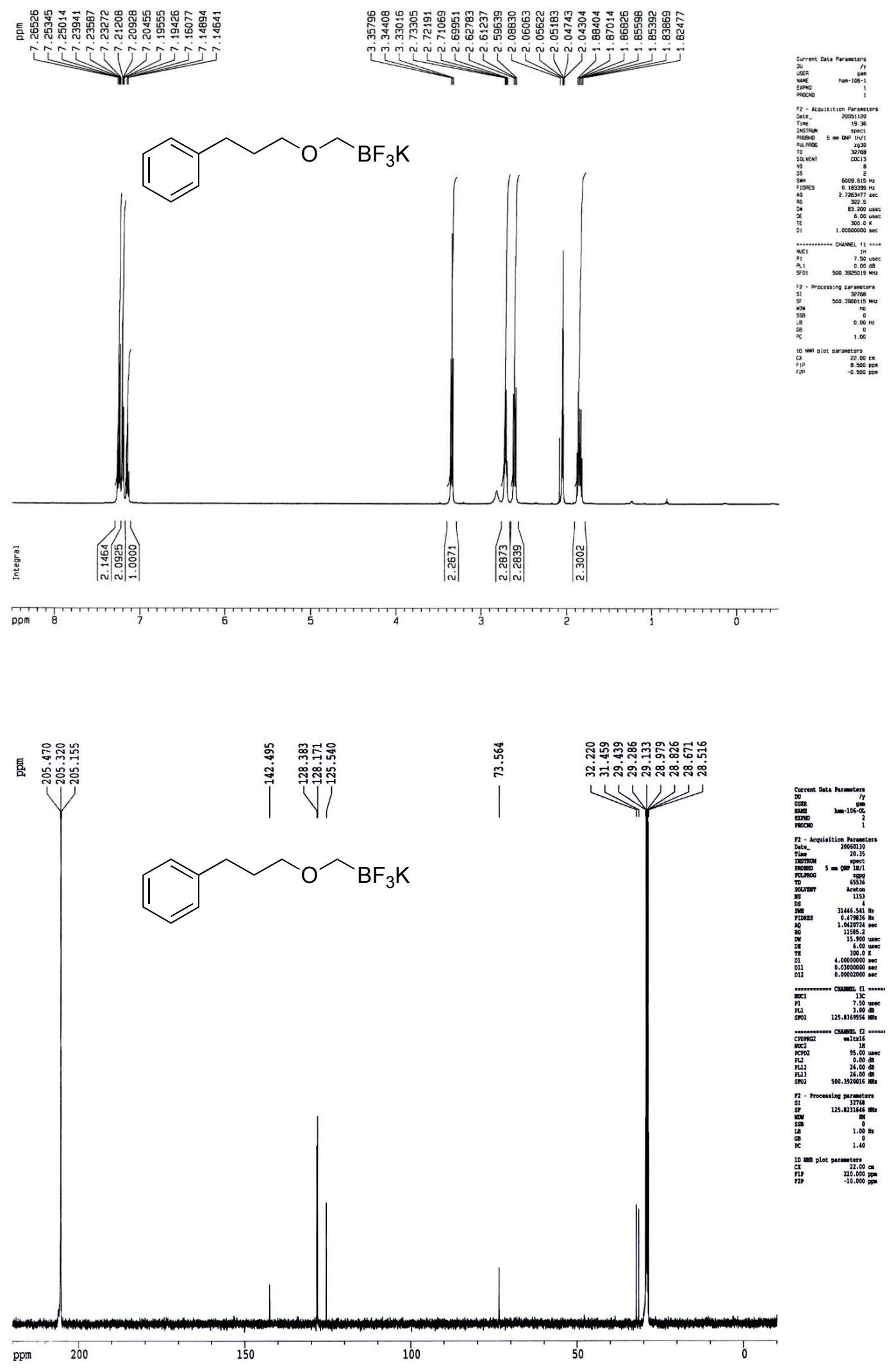

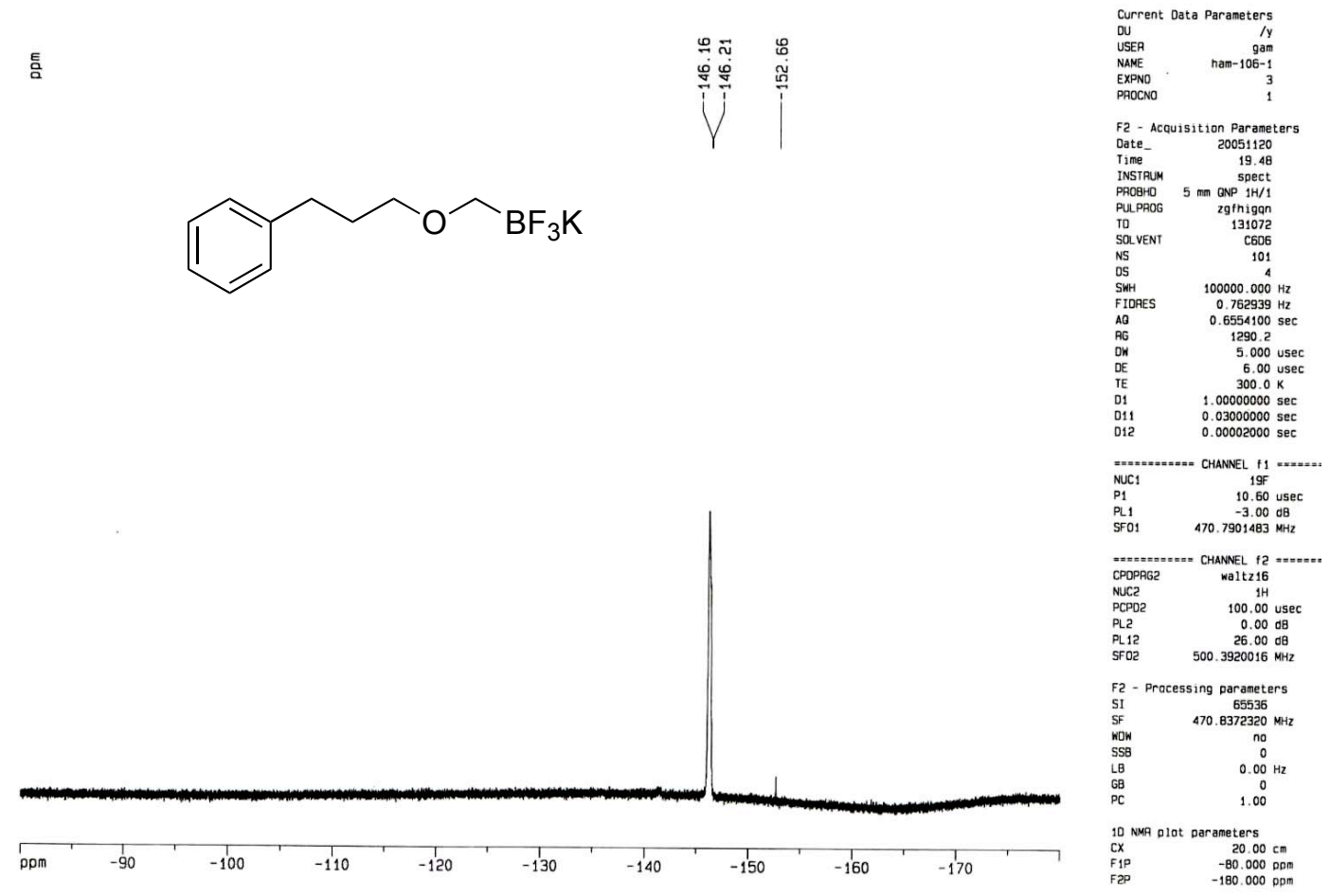

镸
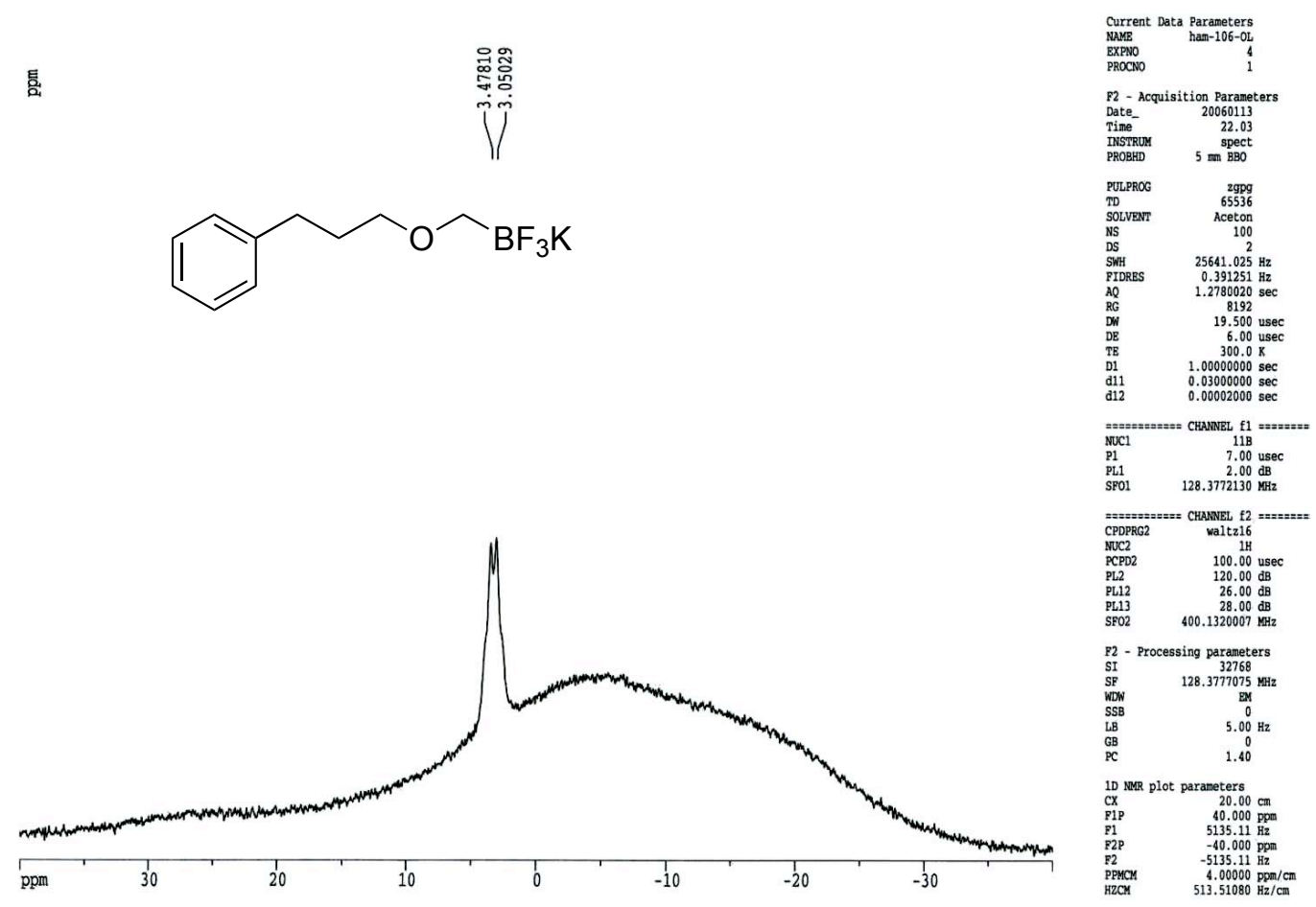

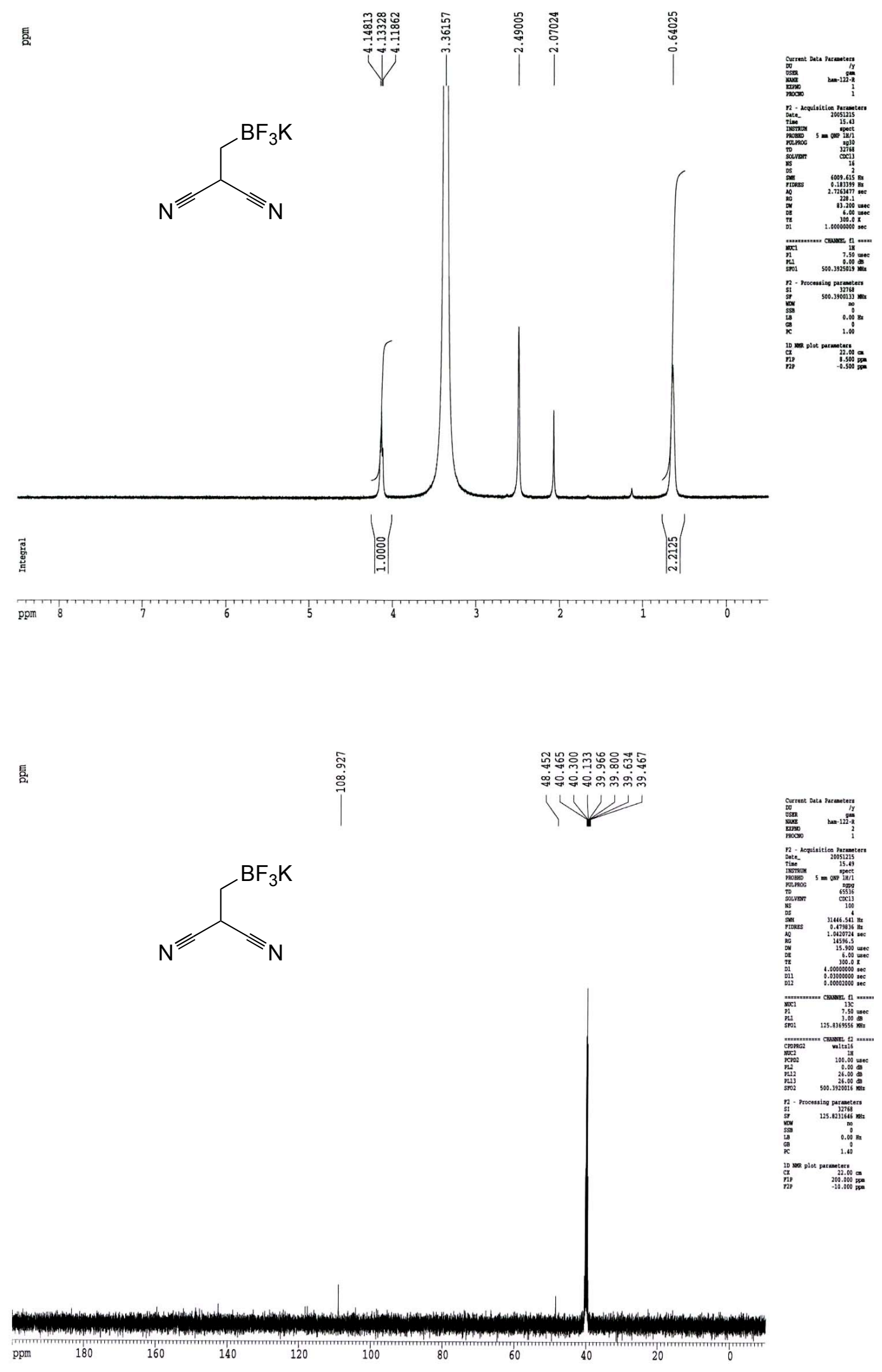

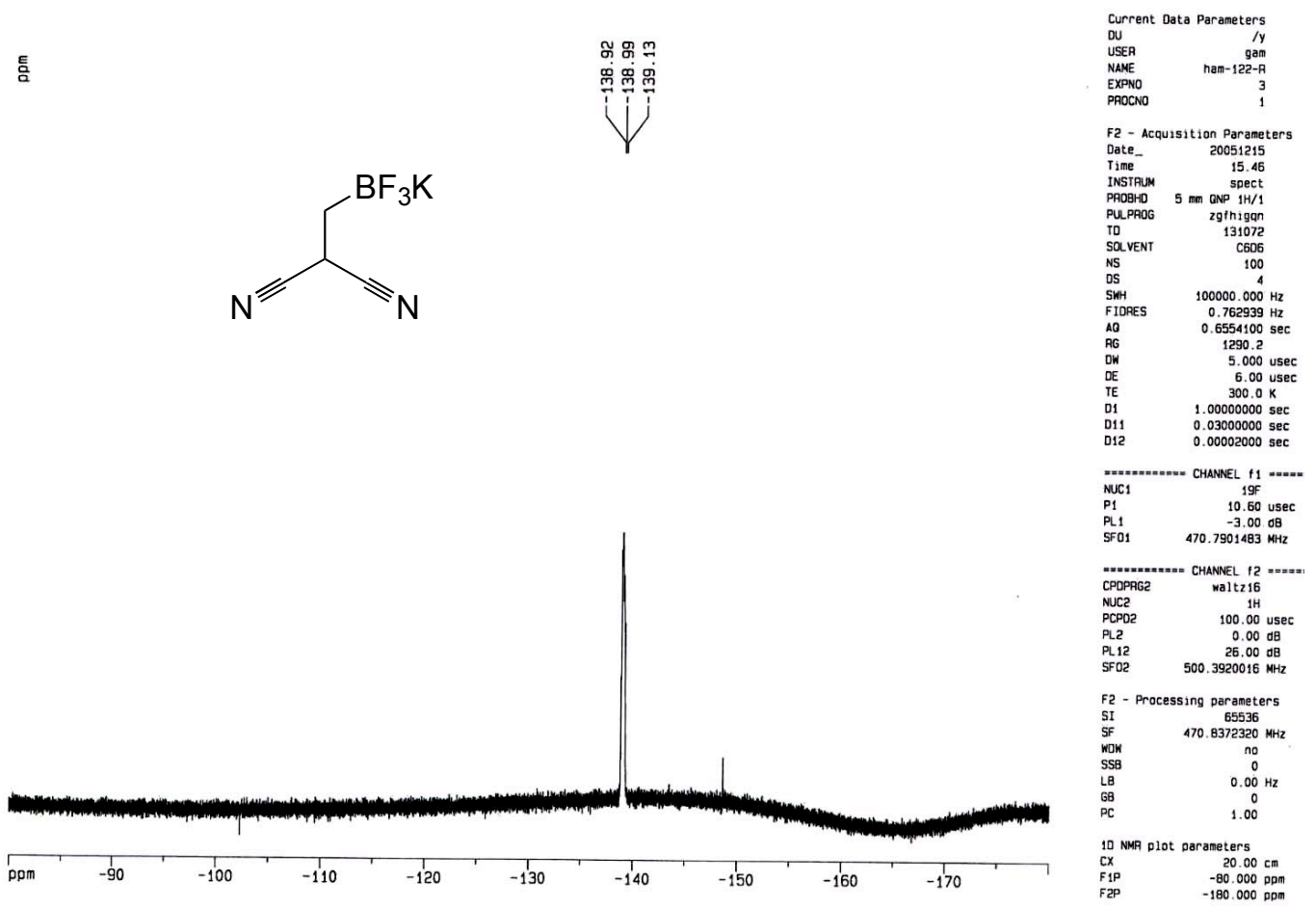

镸

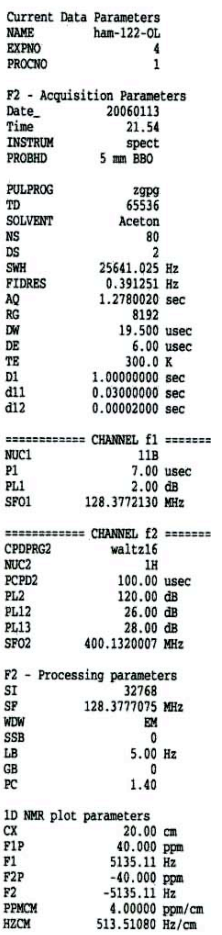


镸
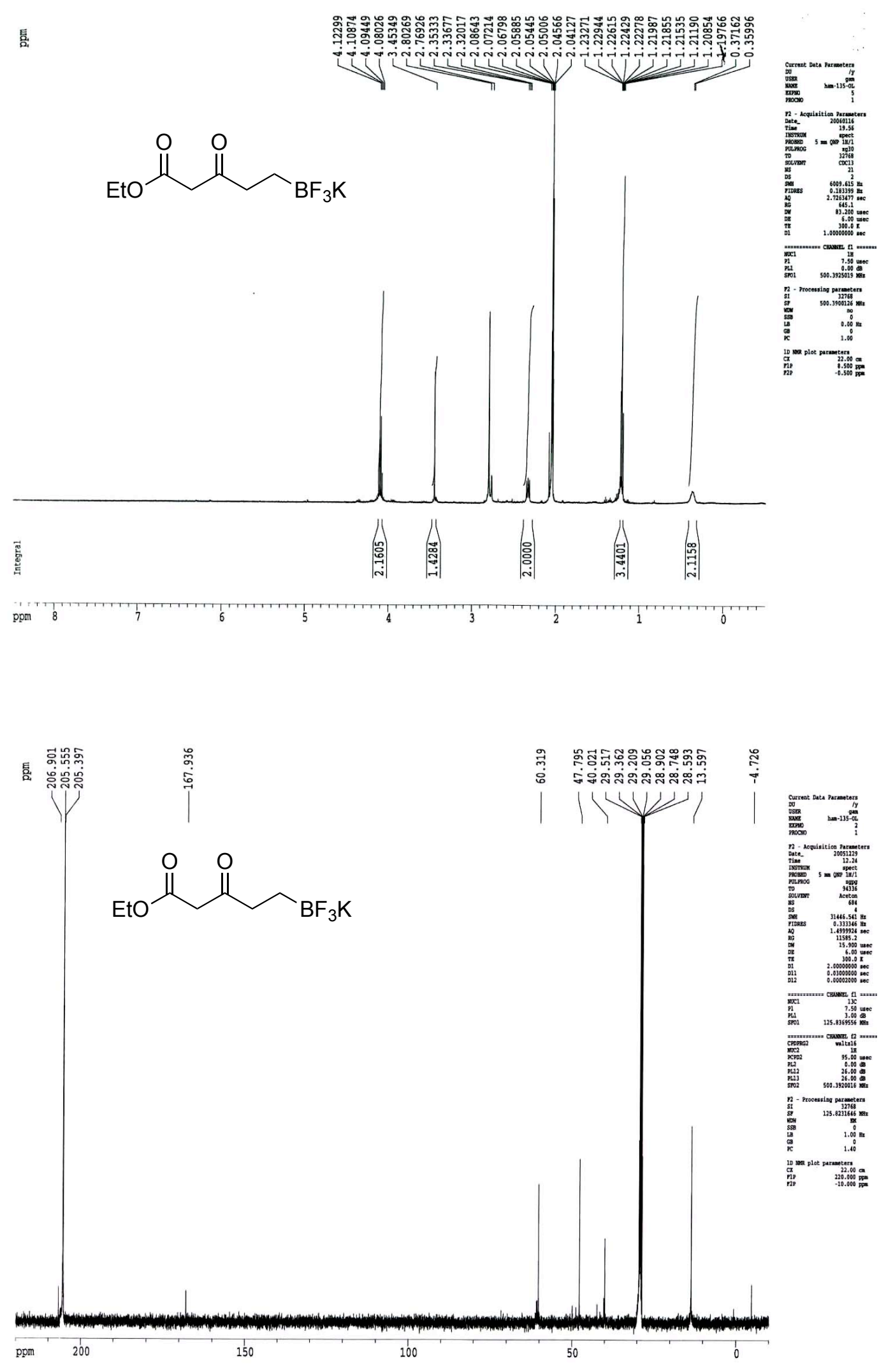


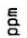

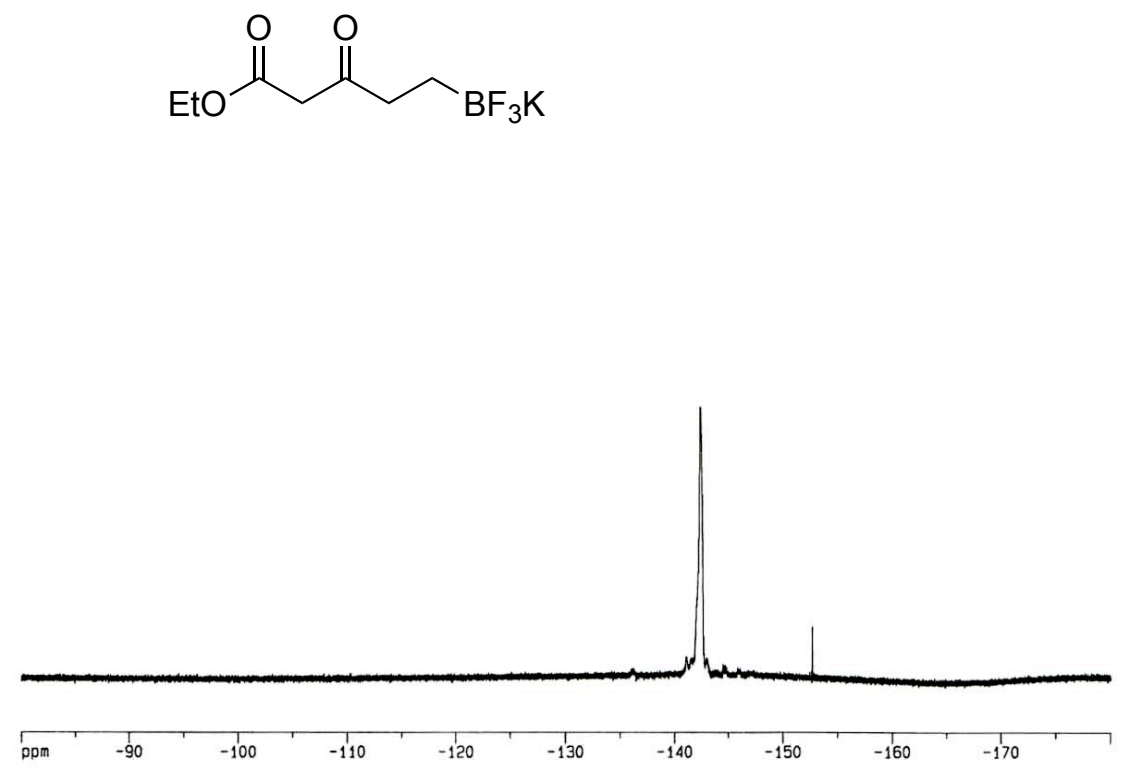

镸
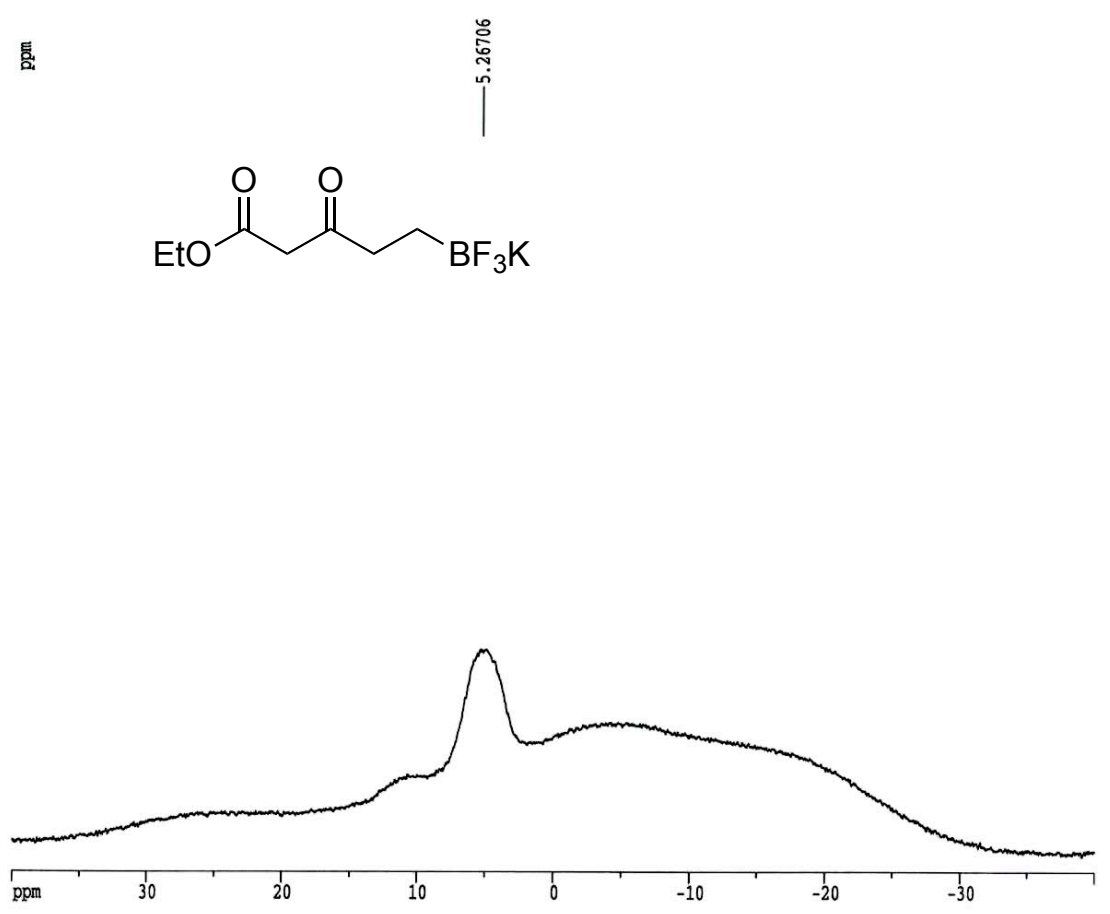

$\begin{array}{lr}\text { Current } & \text { Data Parameters } \\ \text { DU } & \text { ga } \\ \text { USER } & 9 a m \\ \text { NAME } & \text { nan-135-OL } \\ \text { EXPNO } & 3 \\ \text { PFOCNO } & 1\end{array}$

F2 - Acquisition Parameters

$\begin{array}{lc}200512.29 \\ \text { Date_ } \\ \text { Time } & 12.15\end{array}$

Spect
PROBHO $5 \mathrm{~mm}$ ONP $1 \mathrm{H} / 1$

$\begin{array}{lr}\text { PULPPOG } & \text { 2ghiggn } \\ \text { TD } & 131072 \\ \text { Aceton }\end{array}$

\begin{tabular}{lr} 
SOL VENT & Aceton \\
NS & 58 \\
DS & 4 \\
\hline 4 & 00000.000
\end{tabular}

$\begin{array}{rr}\text { ShH } & 100000.000 \mathrm{~Hz} \\ \text { FIDES } & 0.762939 \mathrm{~Hz}\end{array}$

$\begin{array}{ll}A Q & 0.6554100 \mathrm{sec} \\ A G & 1290.2\end{array}$

$\begin{array}{ll}\text { DW } & 5.000 \text { usec } \\ \text { DE } & 5.00 \text { usec } \\ \text { TE } & 300.0 \mathrm{~K}\end{array}$

$1.00000000 \mathrm{sec}$

0.00002000 sec

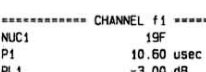

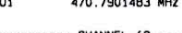

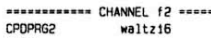

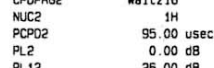
$\begin{array}{rr}\text { PL12 } & 26.00 \mathrm{~dB} \\ \text { SFDO } & 500.3920016 \mathrm{MHZ}\end{array}$

F2 - Processing parameters

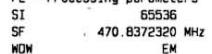
$\begin{array}{lc}\text { NOW } & E M \\ \text { SSB } & 0 \\ L B & 0.30 \mathrm{~Hz} \\ \text { LB } & 0 \\ \text { PC } & 1.00\end{array}$ 10 NMA plot parameters $\begin{array}{lr}C X & 20.00 \mathrm{~cm} \\ \mathrm{FIP}^{2} & -80.000 \mathrm{ppm} \\ \mathrm{F}^{\mathrm{P}} & -180.000 \mathrm{pom}\end{array}$

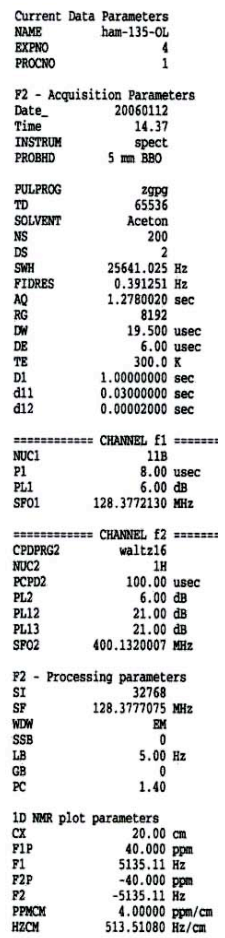



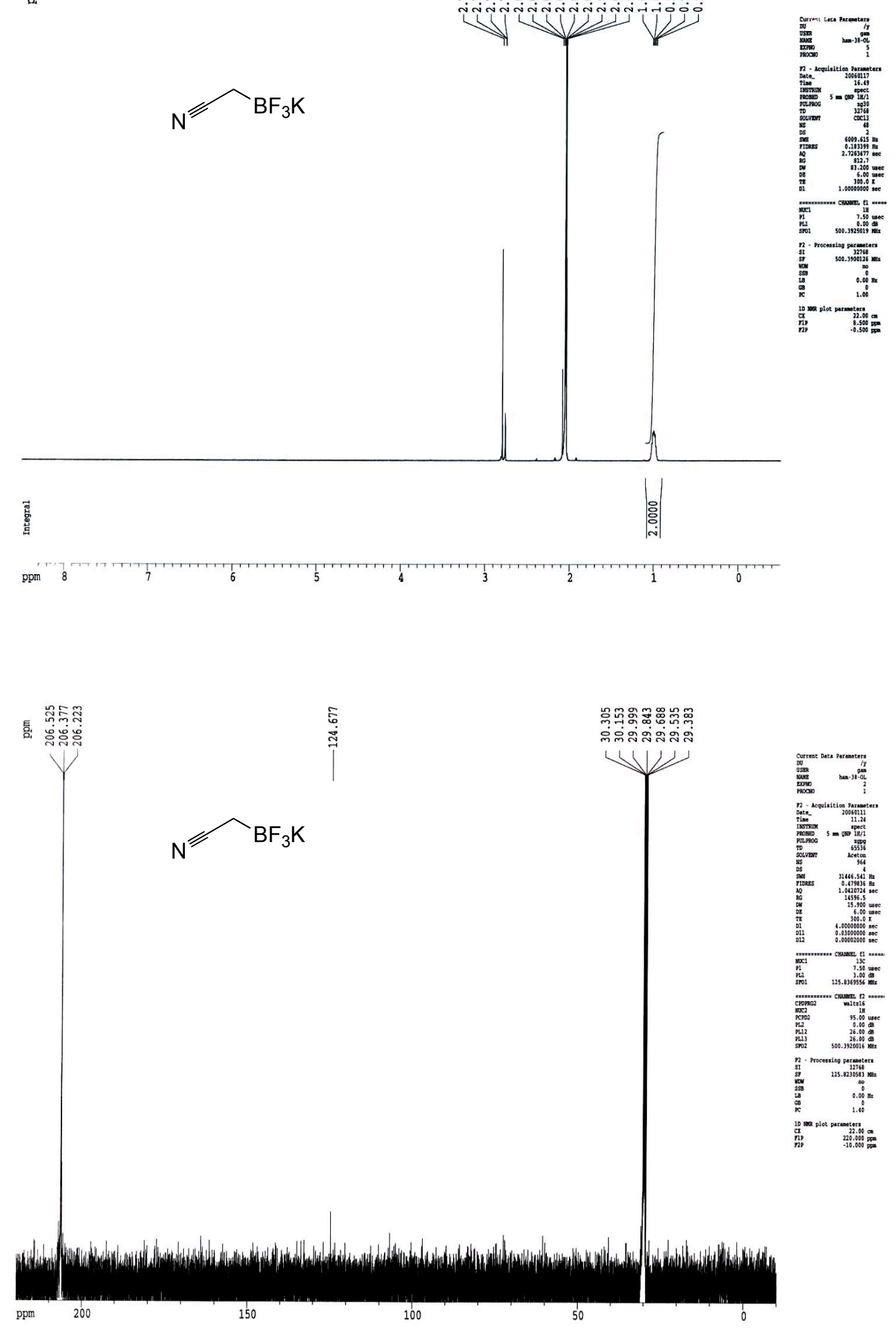

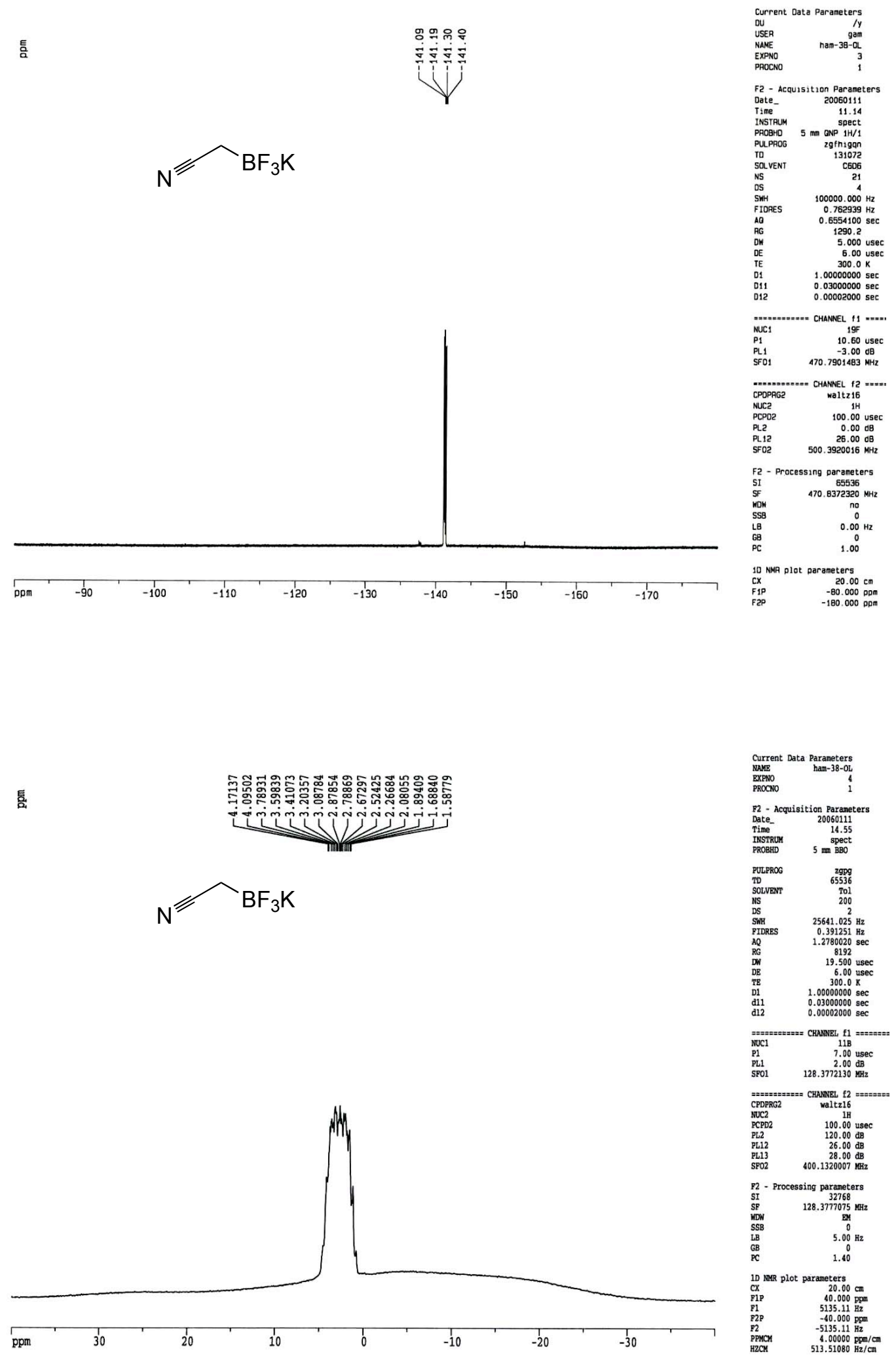


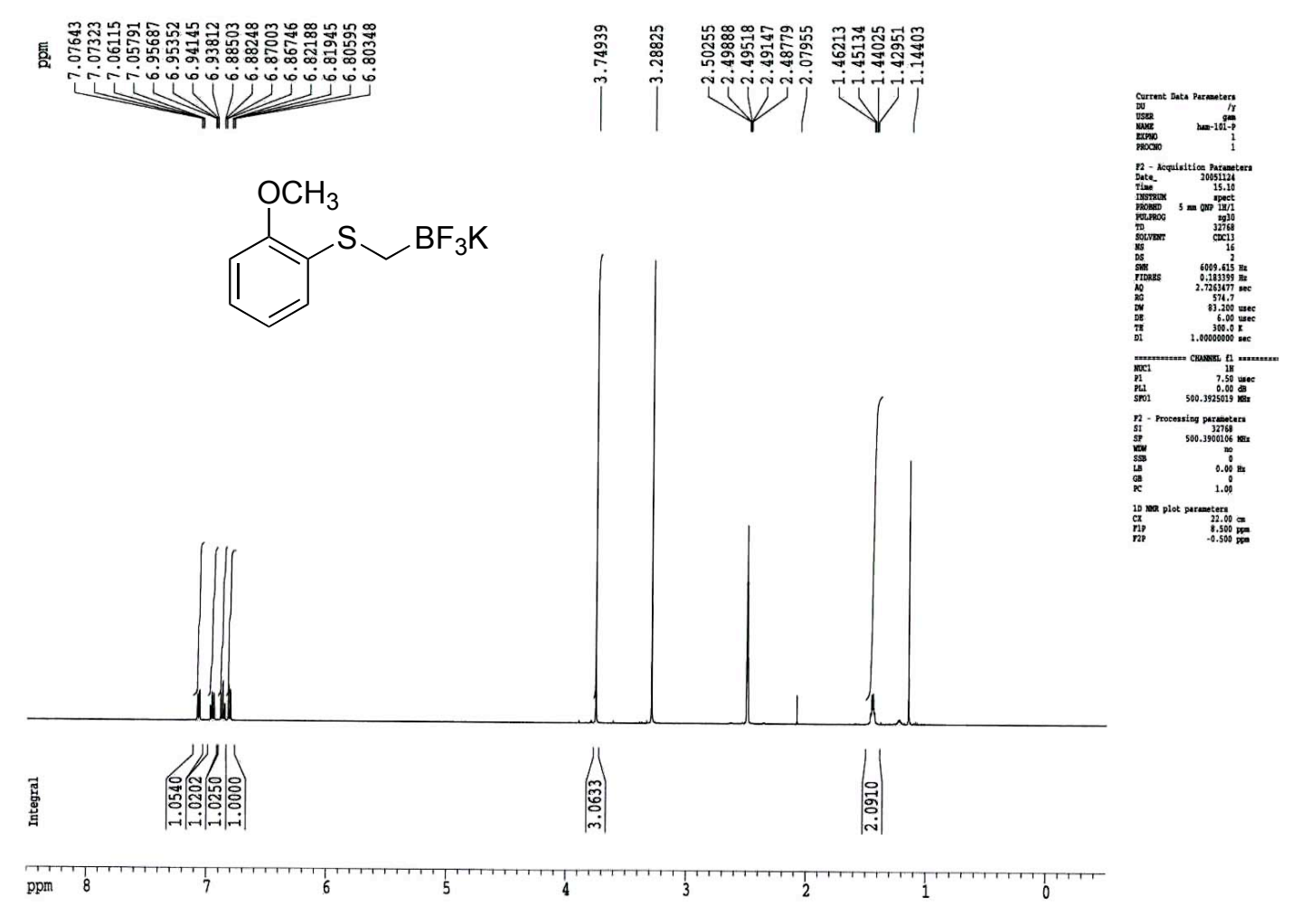

昡
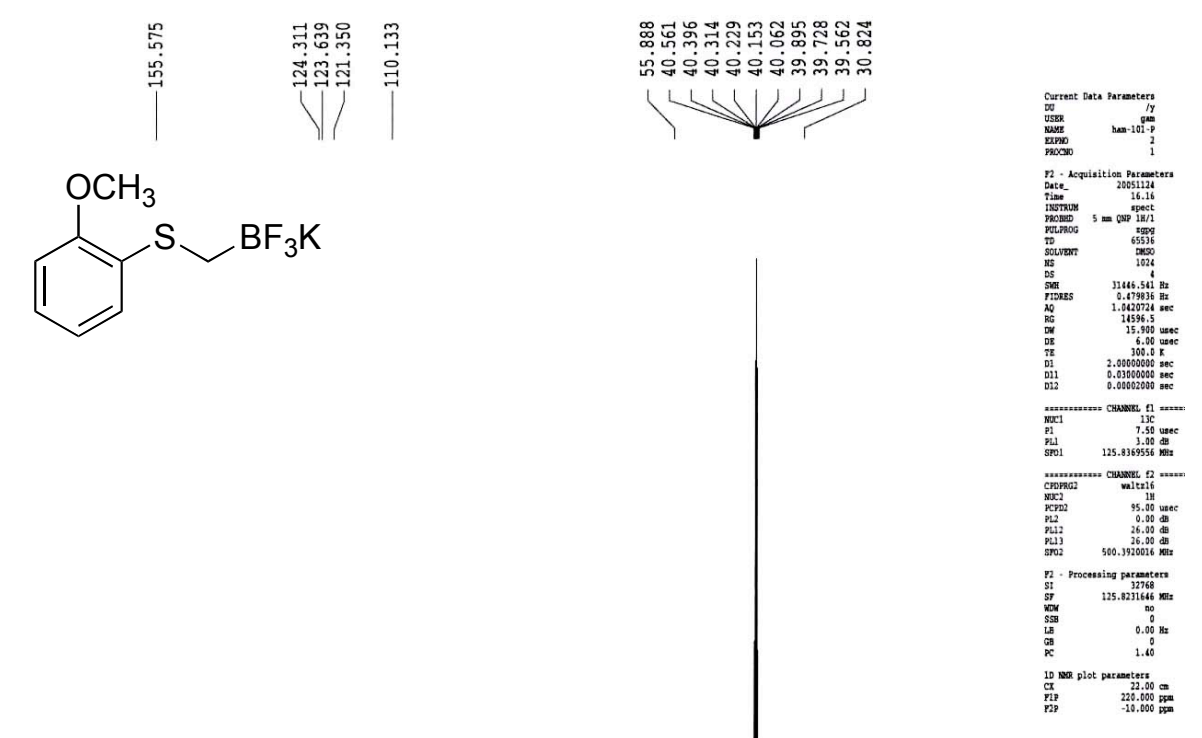

$\mathrm{CHF}_{3}^{\mathrm{OCH}} \sim^{\mathrm{C}}$

ppm 200

${ }_{150}$

100

50

S 36 
公

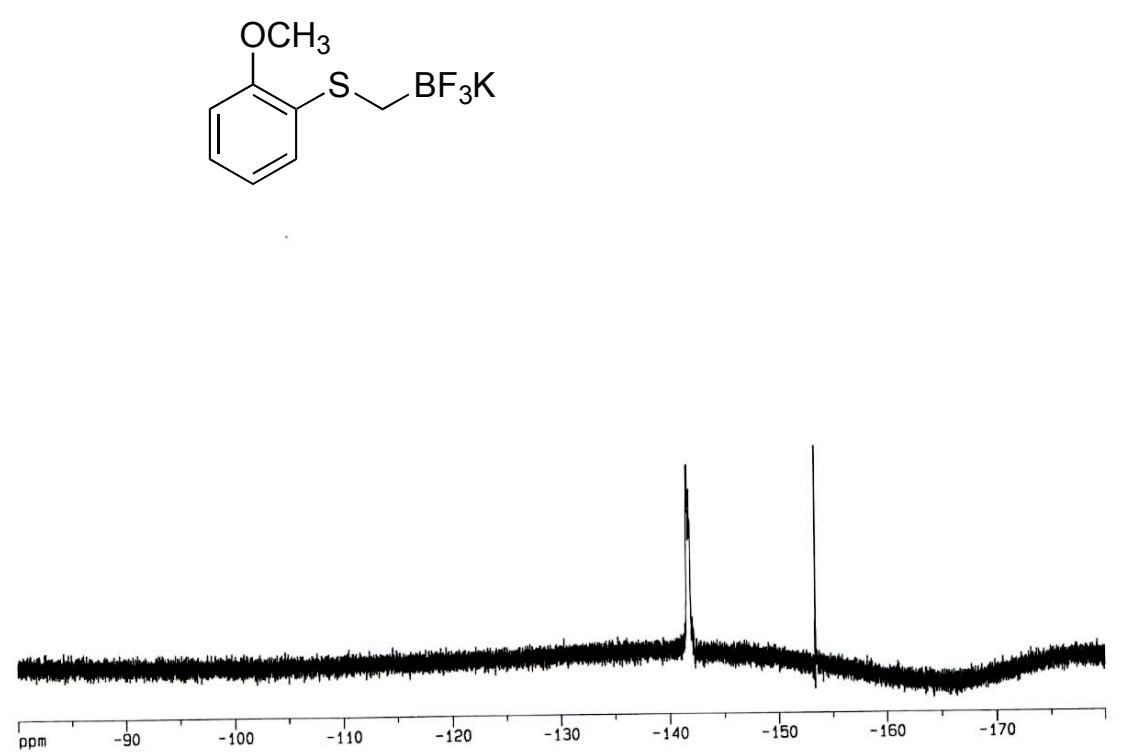

โกิ

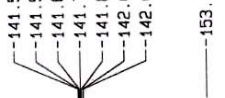

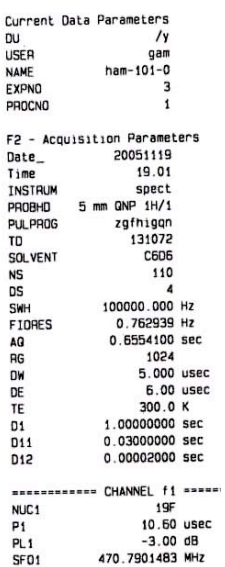

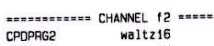

$\begin{array}{ll}\text { NUC2 } & 1 \mathrm{H} \\ \text { PCP02 } & 100.00 \text { usec } \\ \text { PL2 } & 0.00 \mathrm{~dB} \\ \text { PL2 } & 26.00 \mathrm{~dB}\end{array}$

SFO2 500. $3920016 \mathrm{MHZ}$

F2 - Processing parameters

$\begin{array}{ll}\text { SI } & 65536 \\ \text { SF } & 470.8372320 \mathrm{MHz}\end{array}$ $\begin{array}{lc}\text { SSB } & 0 \\ \text { LB } & 0.00 \mathrm{~Hz} \\ G B & 0\end{array}$

10 NMP plot parameters

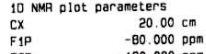

镸

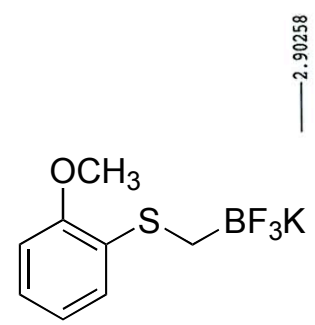

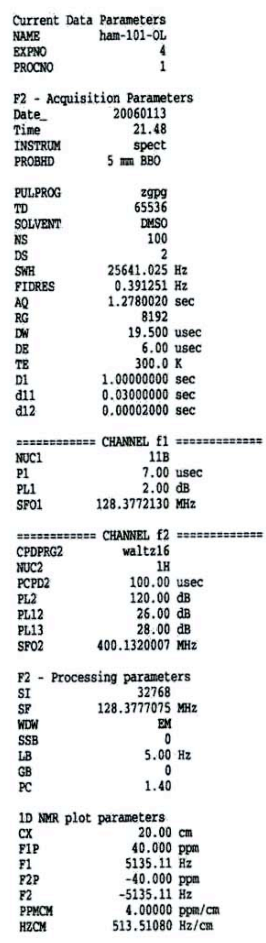



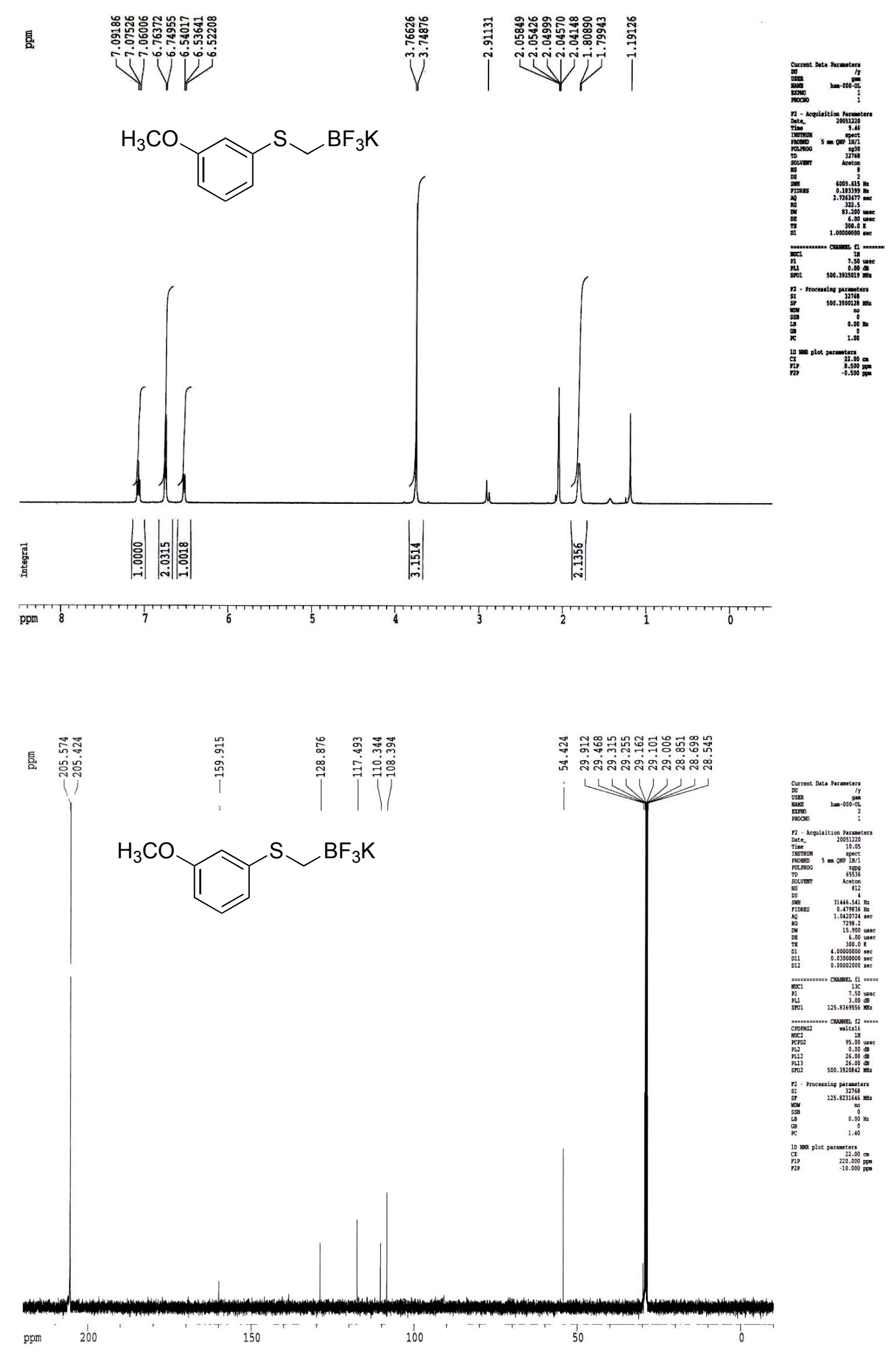

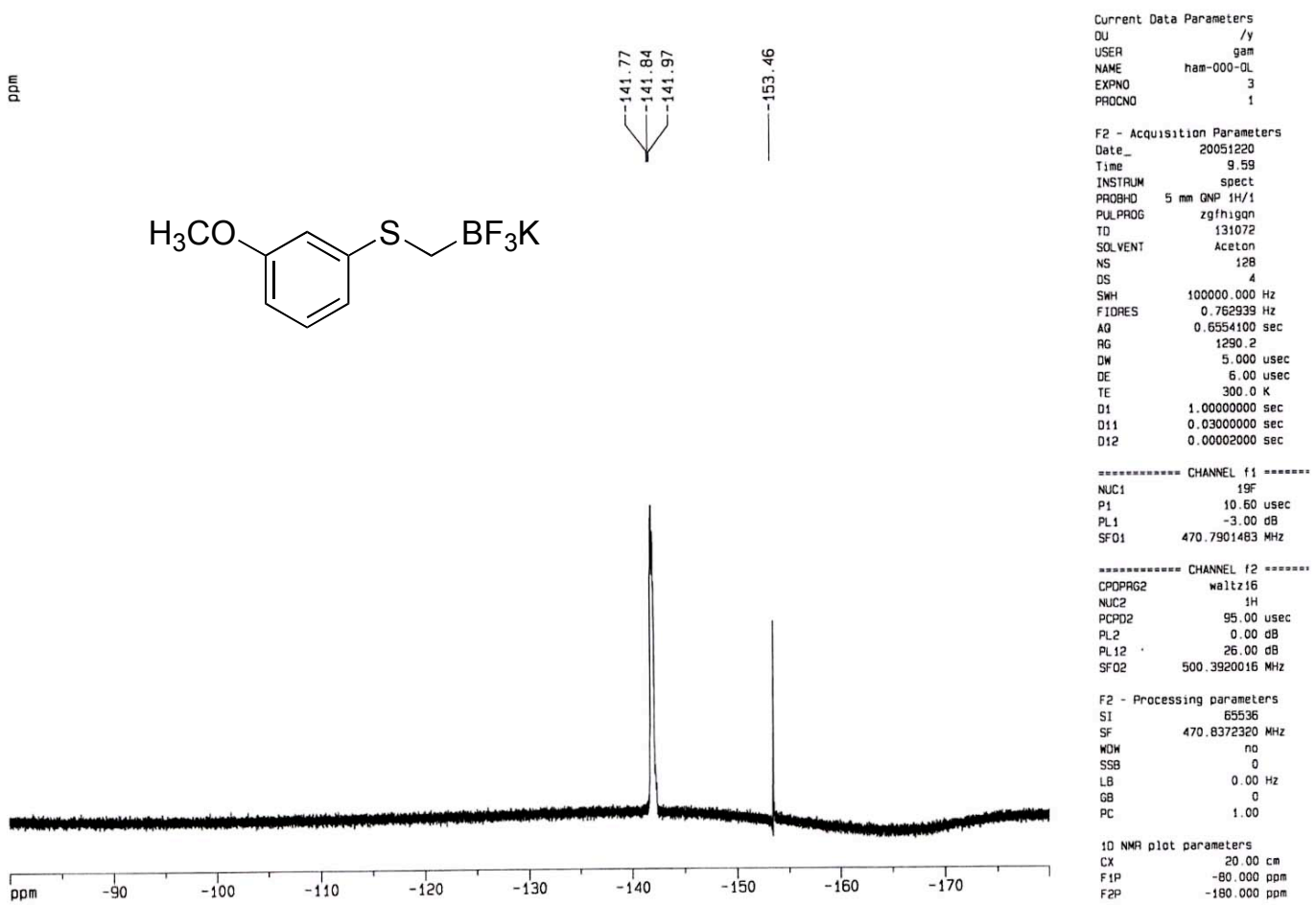

曼

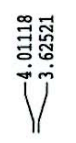

S ${ }^{\mathrm{S}} \mathrm{BF}_{3} \mathrm{~K}$

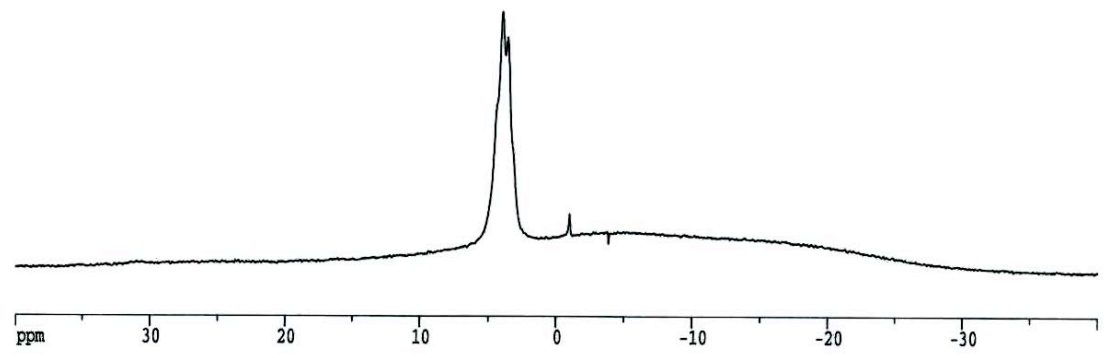

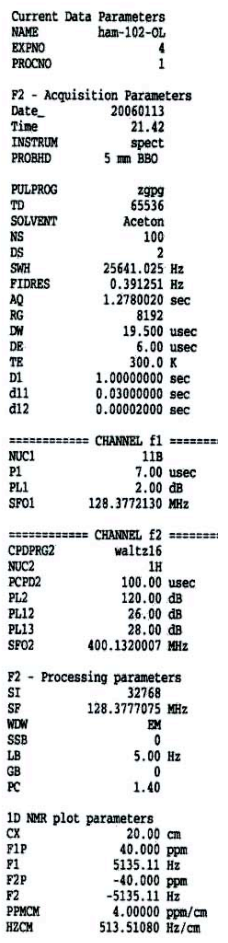



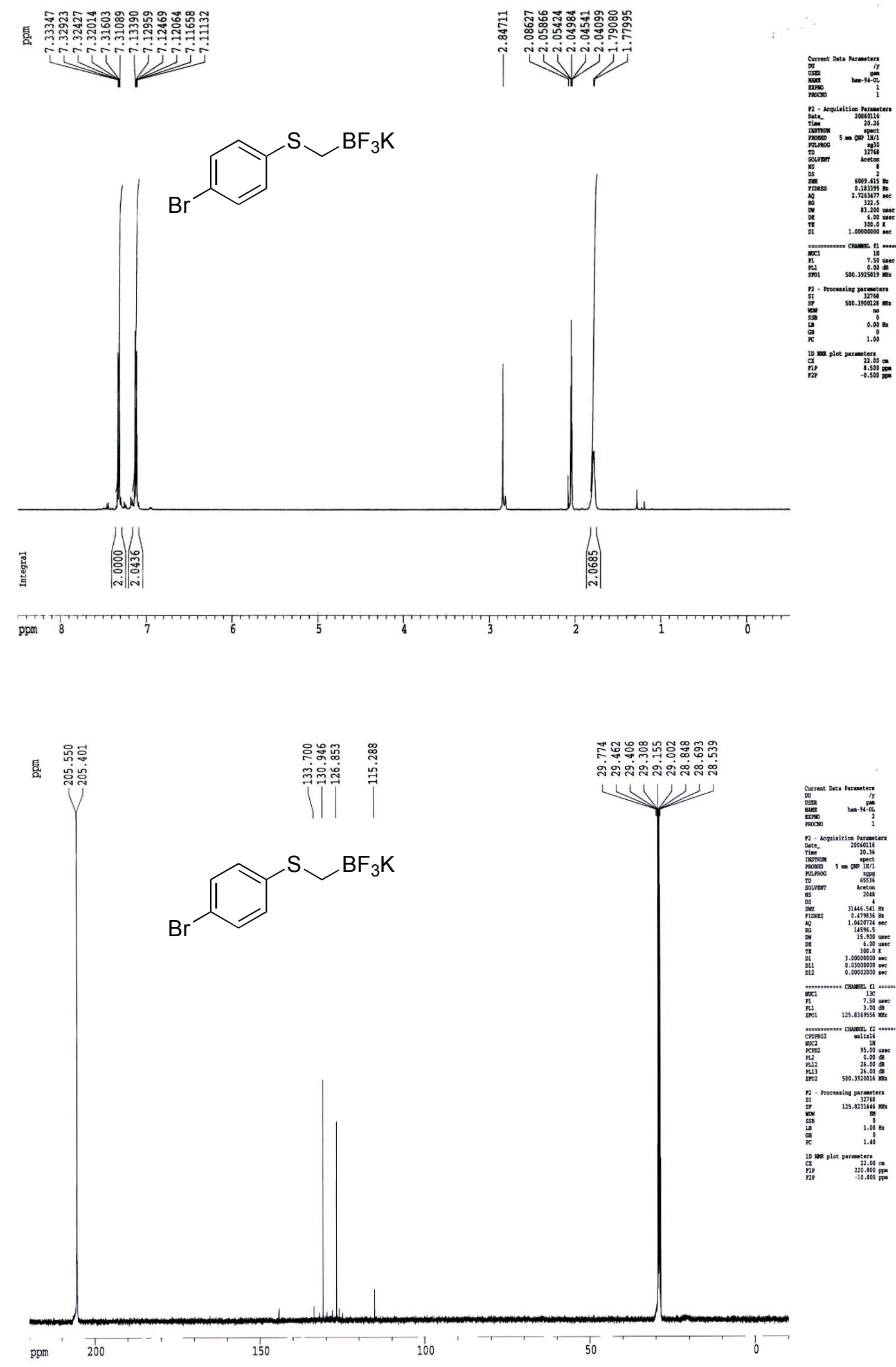
言

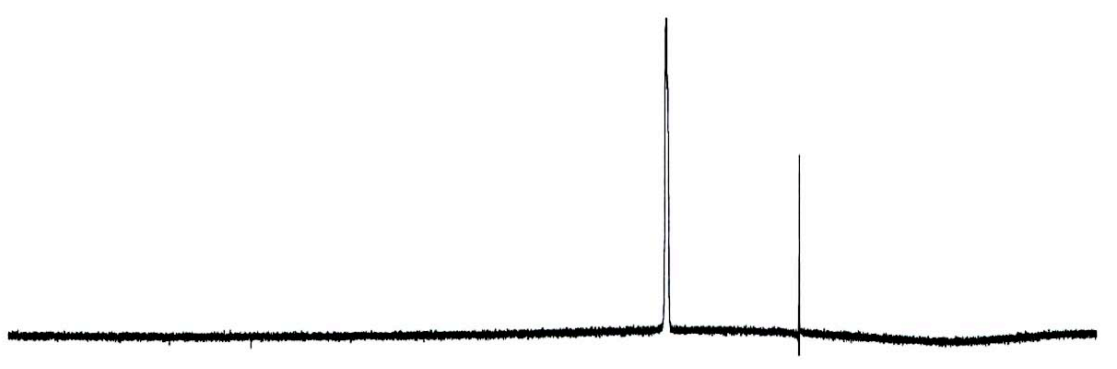

pp<smiles>BrCSc1ccc(Br)cc1</smiles>

$\begin{array}{lr}\text { Current Data Parameters } \\ \text { OU } & / y \\ \text { USER } & \text { gam } \\ \text { NAME } & \text { nam-94-0L } \\ \text { EXPNO } & 3 \\ \text { PROCNO } & 1\end{array}$

F2 - Acquisition Paraneters
Date_ 20060116

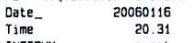

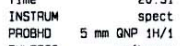

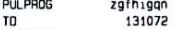

$\begin{array}{lr}\text { SO VENT } & \text { Aceton } \\ \text { NS } & 128 \\ \text { DS } & 4\end{array}$

$\begin{array}{ll}\text { DS } & 4 \\ \text { SnH } & 100000.000 \mathrm{~Hz}\end{array}$

AG $\quad 0.6554100 \mathrm{sec}$

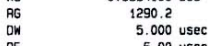

$6.00 \mathrm{usec}$
$300.0 \mathrm{~K}$

.00000000 sec

$0.03000000 \mathrm{sec}$

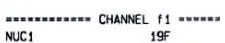

$\begin{array}{ll}\text { NUC1 } & 19 F \\ \text { P1 } & 10.60 \text { usec }\end{array}$

$-3.00 \mathrm{CB}$
$\mathrm{SFO1}$

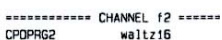

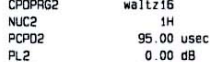

PL2 $\quad 0.00 \mathrm{~dB}$

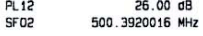

F2 - Processing parameters
SI 6556

$\begin{array}{lc}\text { SF } & 470.8372320 \mathrm{M} \\ \text { WOW } & 00 \\ \text { SSB } & 0 \\ \text { LB } & 0.00 \mathrm{~Hz} \\ \text { GB } & 0.00\end{array}$

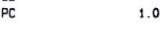

10 NMA plot parameters

$\begin{array}{lr}\text { CX } & 20.00 \mathrm{~cm} \\ \mathrm{~F} 1 \mathrm{P} & -80.00 \mathrm{ppm} \\ \mathrm{FP} & -980.000 \mathrm{ppm}\end{array}$

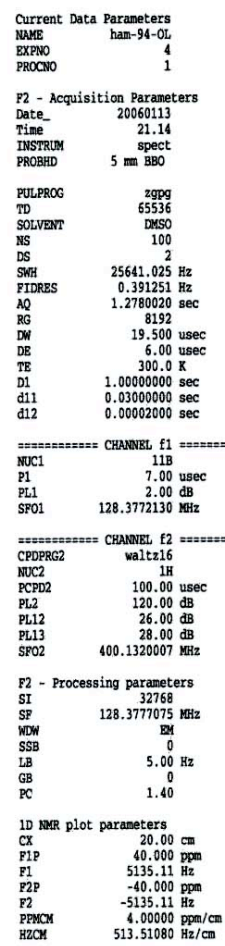




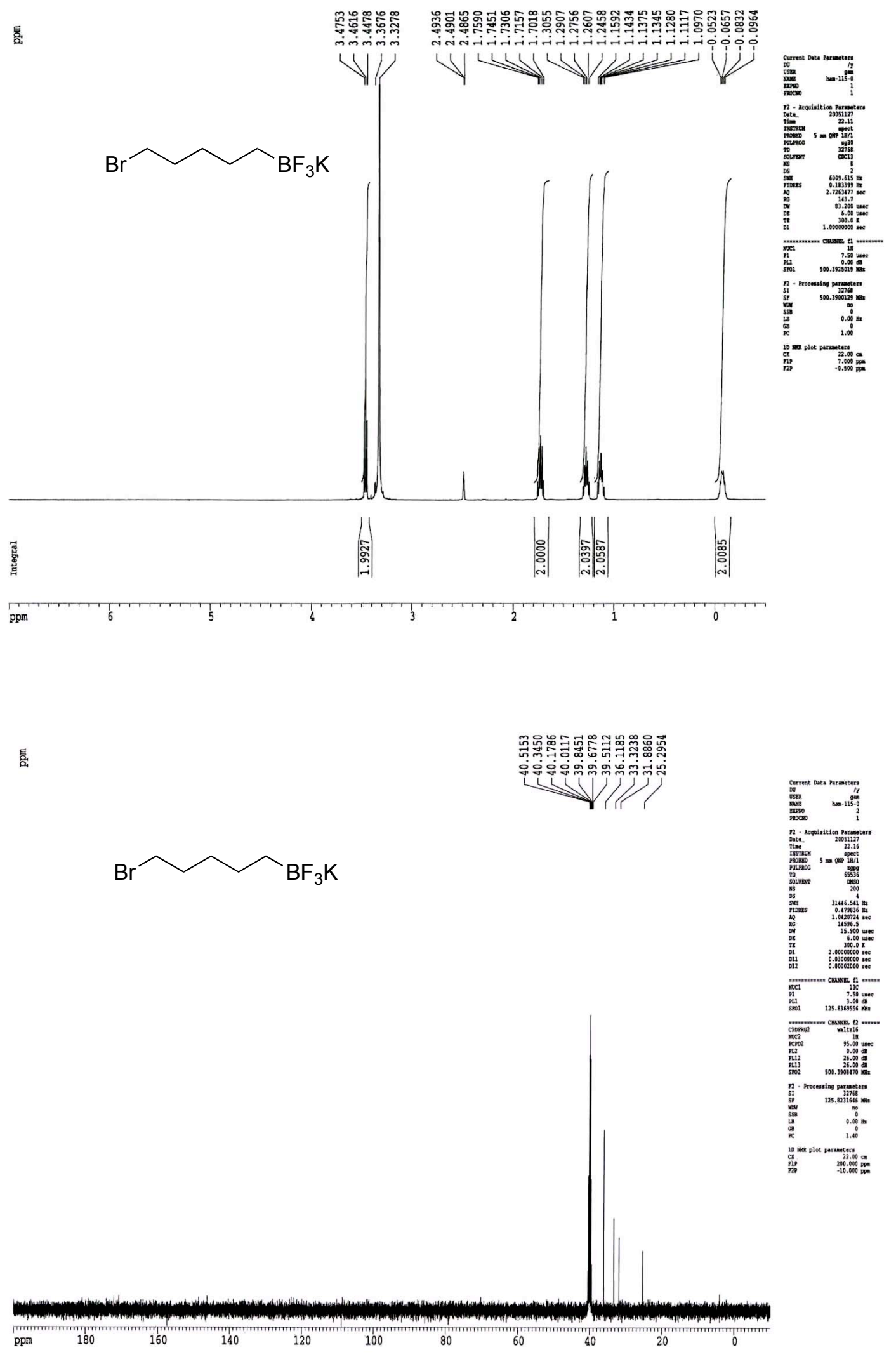



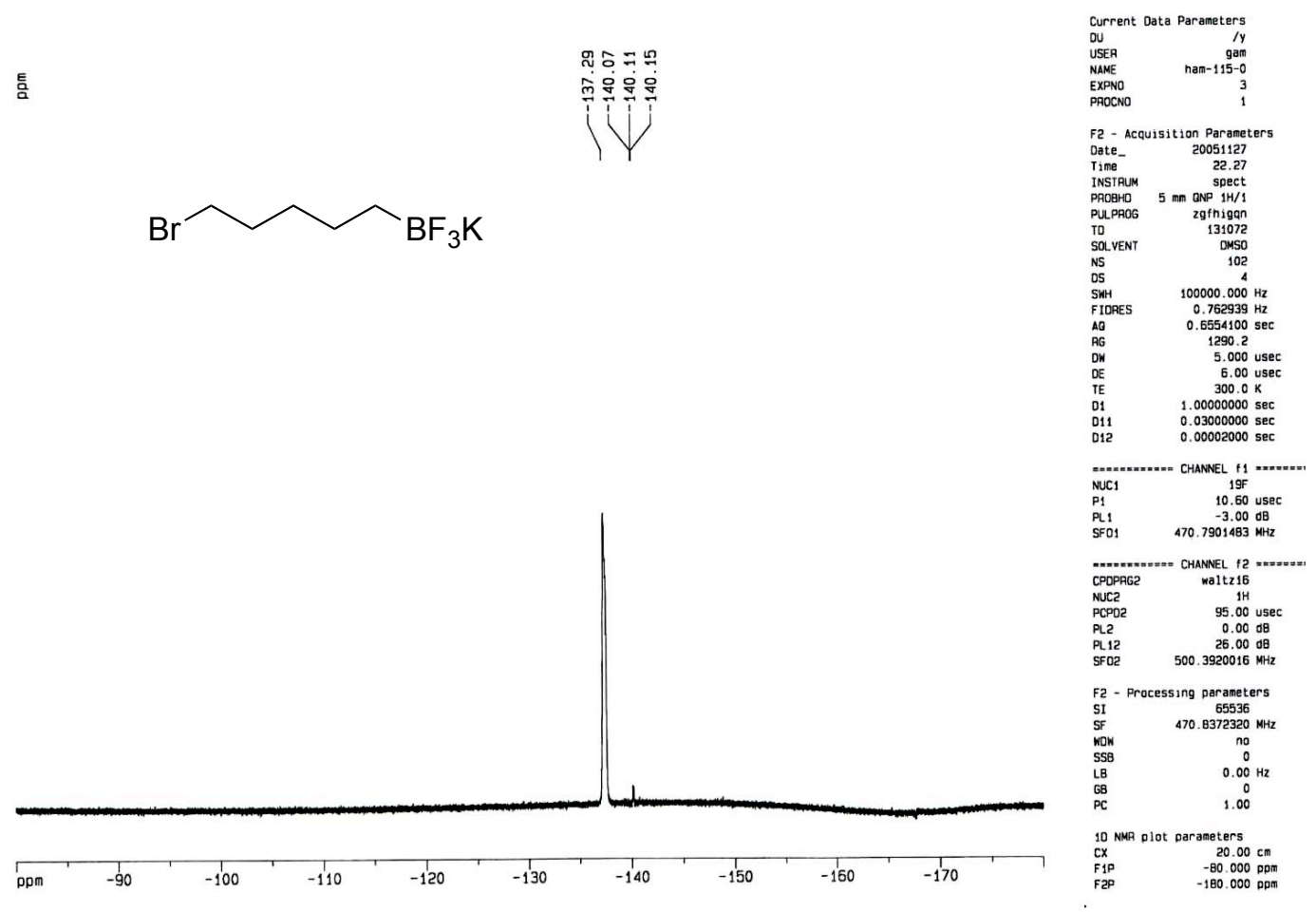

$\begin{array}{lr}10 & -80.000 \mathrm{ppm} \\ \mathrm{F} 2 \mathrm{PP} & -180.000 \mathrm{ppm}\end{array}$
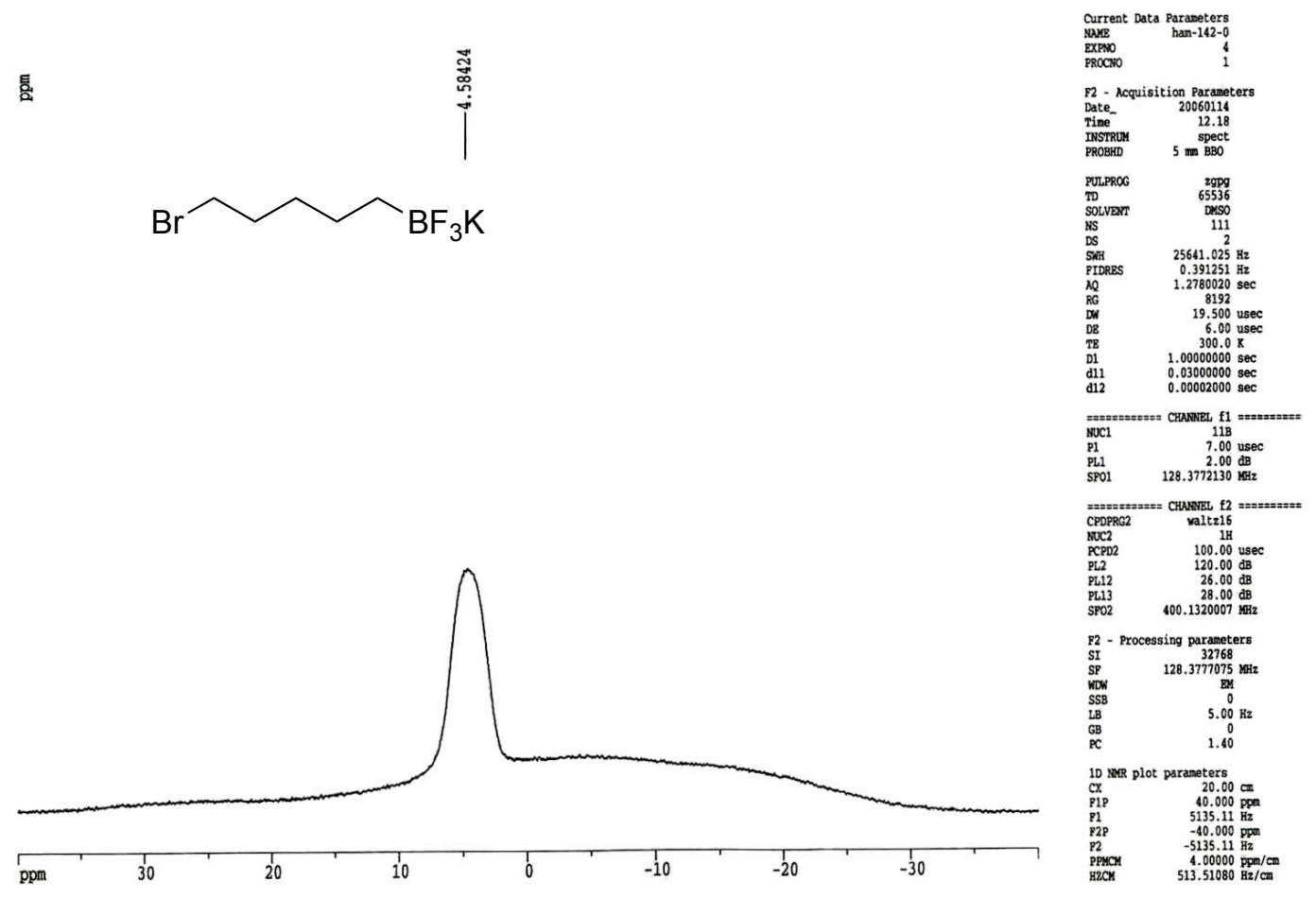

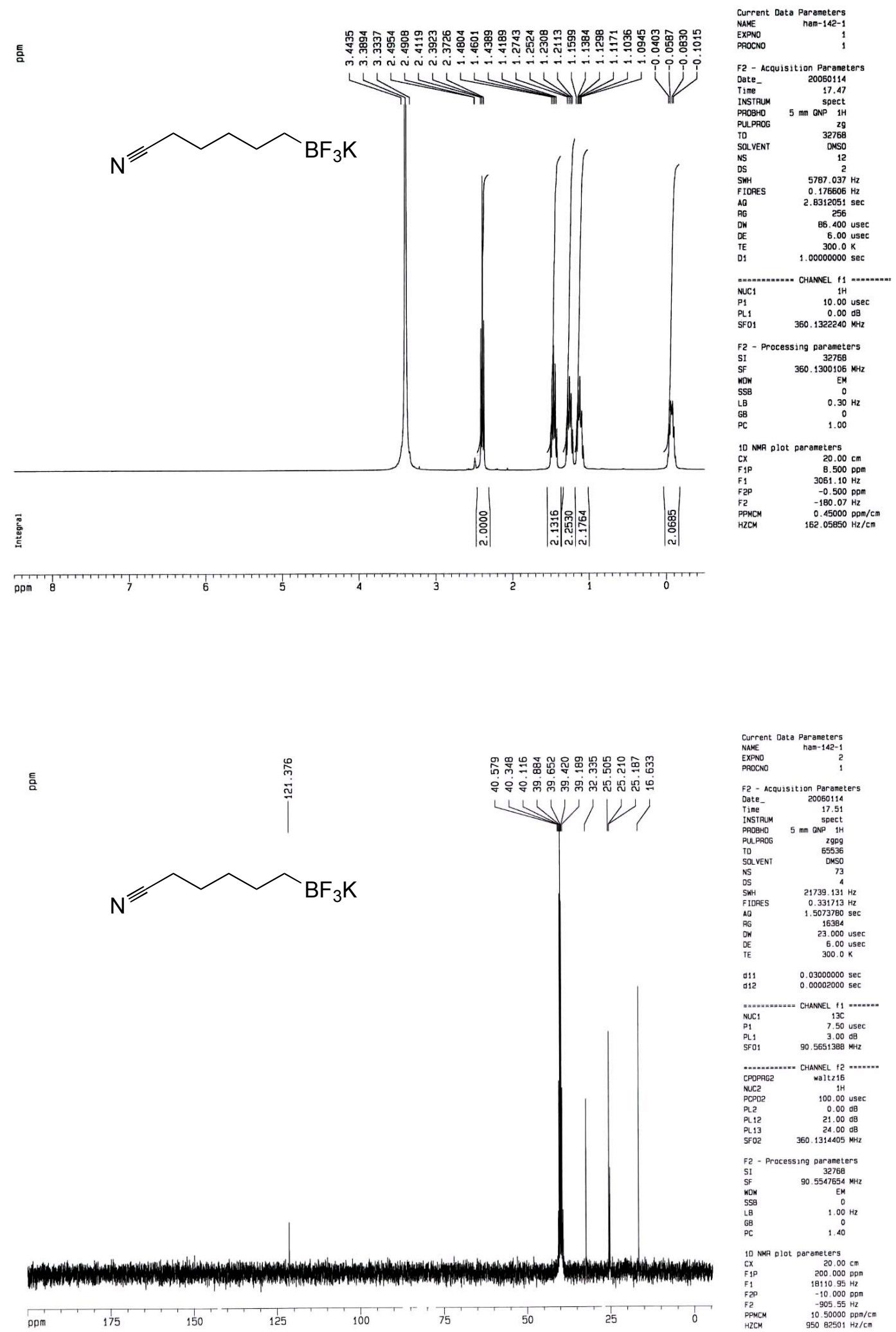

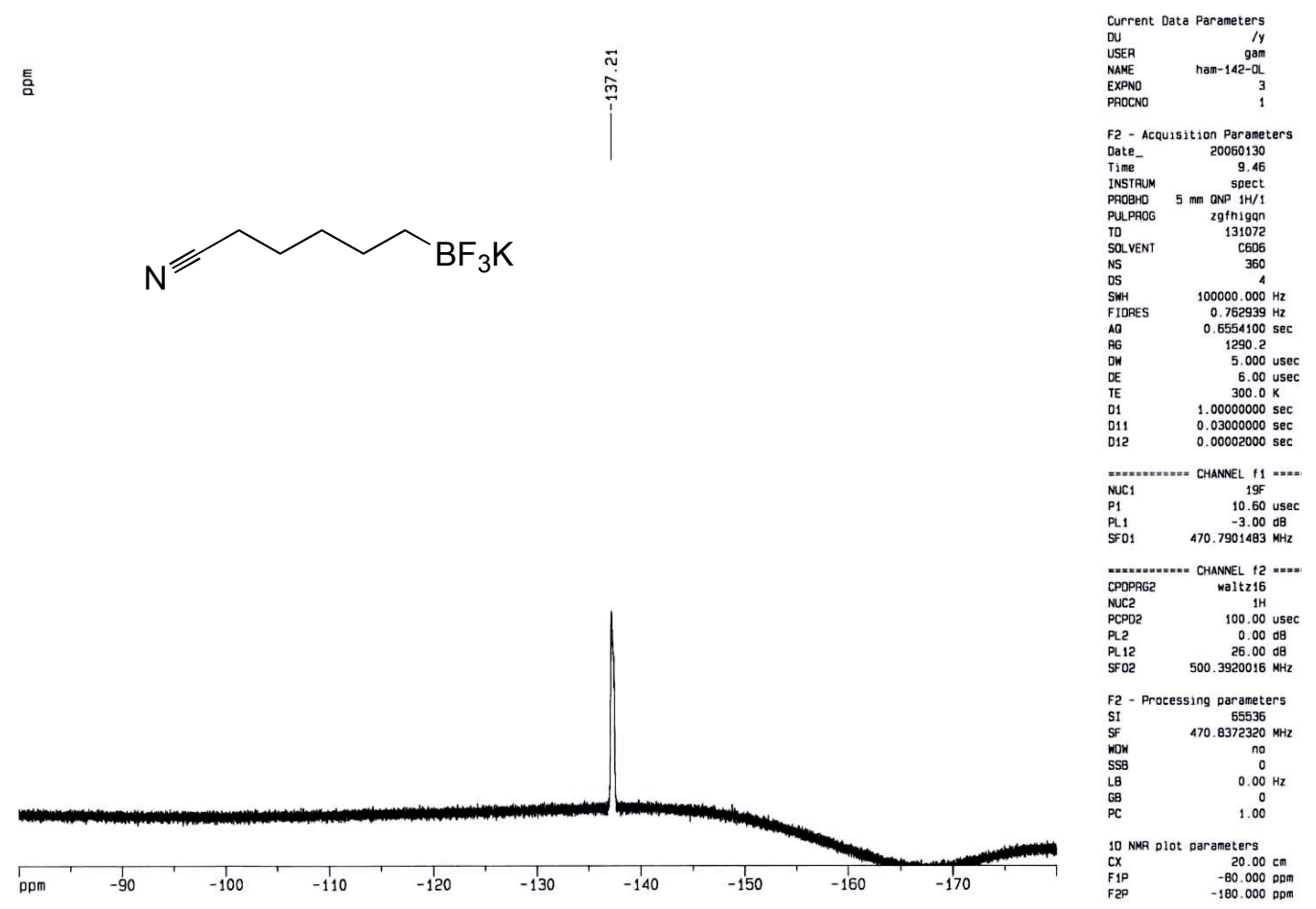

镸
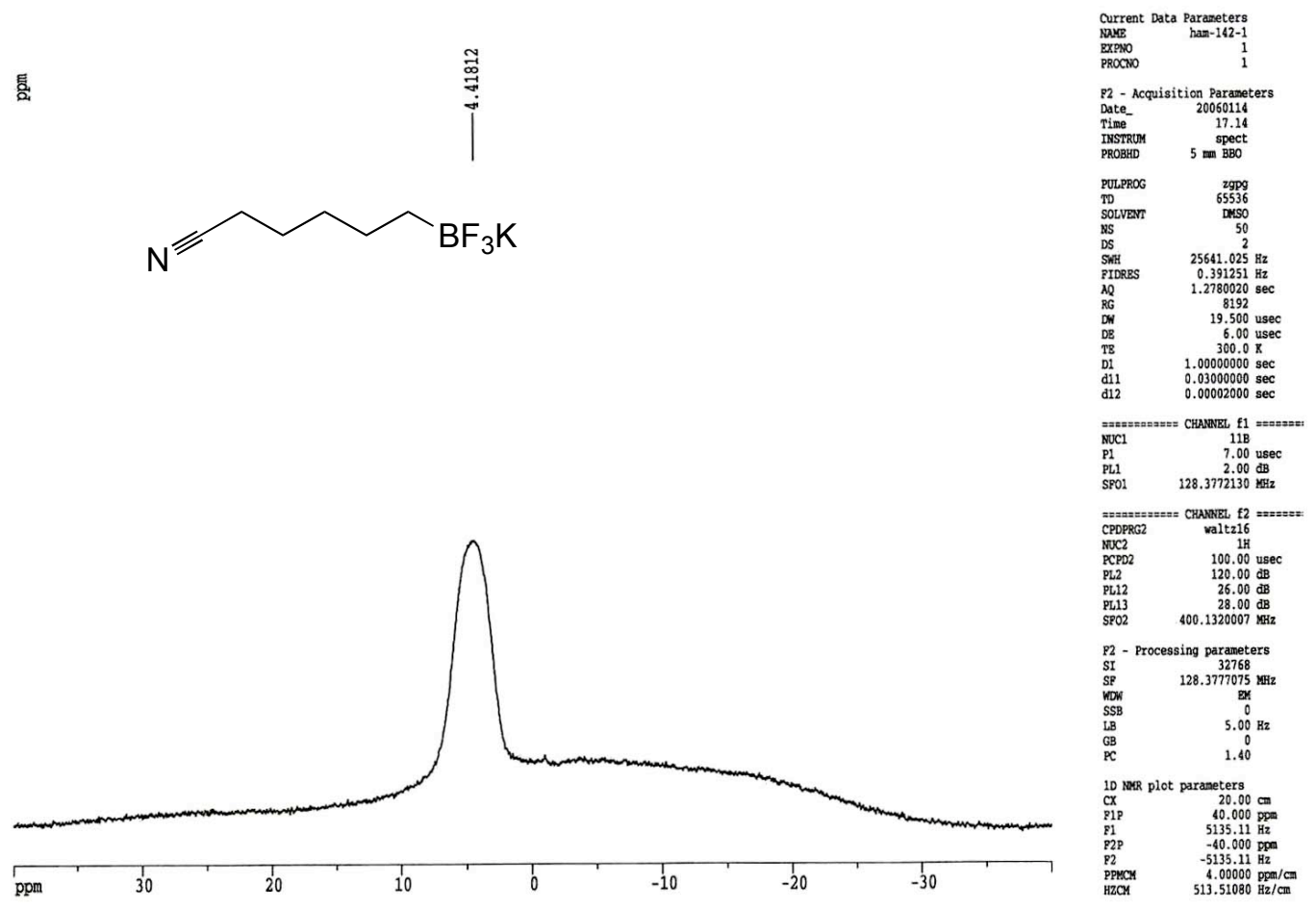